\title{
Topological Horseshoes for Transitive 2-Torus Homeomorphism
}

\author{
Pollyanna Vicente Nunes
}

THESIS PRESENTED

TO THE

Institute of MATHEMATICS AND STATiSTiCS

$\mathrm{OF}$ THE

UNIVERSITY OF SÃo PAUlO

FOR

THE DEGREE

$\mathrm{OF}$

Doctor OF SCIENCE

Program: Applied Mathematics

Advisor: Prof. Dr. Fábio Armando Tal

During the development of this work the author was financially supported by CAPES

São Paulo, June 2021 


\section{Topological Horseshoes for Torus Homeomorphism Topologically Transitive}

This version of the thesis contains the corrections and changes suggested by the Judging Committee during the defense of the original version of the work, held on June 11, 2020. A copy of the original version is available at Institute of Mathematics and Statistics of the University of São Paulo.

Judging Committee:

- Prof. Dr. Fabio Armando Tal (advisor) - IME-USP

- Prof. Dr. Salvador Addas Zanata - IME-USP

- Prof. Dr. Alejandro Kocsard - UFF

- Prof. Dr. Juliana Xavier Saavedra - UDELAR

- Prof. Dr. Alejandro Miguel Passeggi Diaz Robles - UDELAR 


\section{Acknowledgment}

A realização dessa tese de doutorado foi possível graças a ajuda e o apoio, direto ou indireto, de diversas pessoas. À elas (e com medo de cometer a gafe de esquecer alguém), deixo aqui o registro do meu agradecimento.

Ao meu eterno amor, minha 'vó' Norvina (in memoriam), por todo seu amor e dedicação à nossa família e, principalmente, por ter me incentivado a seguir "no caminho dos estudos', como ela dizia. Esse incentivo foi o principal responsável pela minha trajetória acadêmica.

À minha inspiração de força e luta, minha mãe Ely, por todo o seu amor e amparo incondicionais para que eu pudesse alcançar os meus objetivos.

À minha melhor amiga, minha irmã Paola, pessoa com quem eu mais gastei horas ao telefone durante esse período, que me aguentou nos meus piores dias, me ouviu, aconselhou e foi o meu refúgio.

Ao meu pai Nunes, por sempre estar na rodoviária me esperando chegar ou me vendo partir em todas as minhas idas e vindas de São Paulo.

Aos meus afilhados, Miguel e João Gabriel, e minha sobrinha, Manuela, que são a minha fonte de alegria e para quem eu quero servir de inspiração.

À minha prima Mikaella, por me ouvir, aconselhar e ser a pessoa da família que mais se parece comigo.

À minha duplinha Thiara e Isabela, por serem meu porto seguro, por me proporcionarem os melhores (re)encontros em Itaperuna e por despertarem a minha versão menos formal: aquela que gosta de ver e comentar os reality shows, que gosta de festa e funk, que faz careta pra virar meme e figurinha de whatsapp, e tantas outras coisas 'bobas', mas que fazem a minha vida mais feliz.

Às minhas princesinhas de Itajubá, Lívia, Clara, Marina e Juliana, por terem sido minha válvula de escape do caos de São Paulo, por rirem das minhas piadas, por serem as principais admiradoras dos meus dotes culinários e, principalmente, por estarem sempre comigo, me dando força e torcendo por mim.

À minha amiguinha Jerusa, por ter sido uma irmã em São Paulo e por ter vivido comigo as mais diversas situações: árduas horas de estudo na salinha de doutorado (e em casa), bloquinhos de Carnaval por São Paulo, um dia inteiro comigo num hospital passando mal, algumas (lê-se: várias) noites de happy hour na nossa casinha e tantos outros momentos que vão ficar marcados pra sempre. 
Às amigas que o IME/USP me deu, Bruna, Larissa e Ana, por além de serem minhas companheiras de estudo, café, bandejão e alguns happy hours, sempre tiveram o conselho certo para me dar, seja sobre a vida pessoal ou acadêmica/profissional.

Ao casalzão Verônica e Vanderli, pela amizade desde a graduação na UFES, por termos vivido juntos o sonho de estar fazendo doutorado nas nossas respectivas áreas e pelas melhores experiências culinárias que três doutorandos podem vivenciar, exceto pela batata de carinha.

Às minhas roomies Marina, Isadora e Aleja, que junto com a Verônica e a Jerusa, me proporcionaram a melhor experiencia de ap compartilhado em São Paulo.

Ao meu amigo Everton, por todo tempo que dedicou estudando comigo a teoria de forcing e pelas palavras de encorajamento.

Ao grande amigo que o IME/USP me deu, Nelson, por sempre estudar comigo, me ajudar a entender coisas 'triviais' e, principalmente, por me acalmar nos momentos de crise.

À minha grande amiga Luciana, por ter sido luz no meu caminho nos primeiros anos de IME/USP e pela amizade e carinho que compartilhamos até hoje, apesar da distância.

Aos companheiros da salinha do doutorado pelas diversas horas de estudo que compartilhamos e pelos bate-papos na salinha do café, no bandejão, nas idas e vindas da USP, etc.

Ao Guilherme, pela amizade, conselhos e momentos de descontração com muita comida e música no 'Jambalaya' e por ter me apresentado a famosa farofa de banana da dona Judith.

Aos amigos que fiz durante as disciplinas e grupos de estudos no IME/USP.

Aos amigos da graduação na UFES e do mestrado na UNIFEI, que mesmo com a distância e pouca comunicação, são igualmente importantes pra mim e minha trajetória.

Aos professores da UFES, da UNIFEI e do IME/USP por todo ensinamento.

Ao professor Fábio Tal, pelo orientação neste trabalho que, mesmo com os mais variados contratempos, a fez com muita maestria e muita paciência, principalmente nos meus momentos de autossabotagem e crises de ansiedade.

À CAPES, pelo suporte financeiro.

E, à mim, que mesmo com toda essa ajuda e apoio recebidos, a decisão de desistir ou não sempre esteve em minhas mãos e eu nunca cedi à ela. Então, muito obrigada Pollyanna, por ter sido corajosa e vencido todo medo e ansiedade que por diversas vezes tomou conta dos seus pensamentos e do seu coração. 


\section{Abstract}

NUNES, P. V. Topological Horseshoe for transitive 2-torus homeomorphism. 2021. 81 f. Tese (Doutorado) - Instituto de Matemática e Estatística, Universidade de São Paulo, São Paulo, 2021.

Based on rotation theory and forcing theory for transverse trajectories of surface homeomorphisms, in this work we study the relation between trasitive homeomorphism of 2-torus, $f: \mathbb{T}^{2} \rightarrow \mathbb{T}^{2}$, and the existence of a topological horseshoe.

In the case where $f$ is isotopic to identity, we prove that $f$ has a topological horseshoe, if $f$ has a fixed point and a non-fixed periodic point.

In the case where a power $f^{k}, k>1$, is isotopic to identity but $f$ itself is not, we show that if $f$ has at least one fixed point and has no topological horseshoe then the rotation set of some lift $\check{f}: \mathbb{R}^{2} \rightarrow \mathbb{R}^{2}$ of $f$, denoted by $\rho(\check{f})$, is only the origin.

We also study the case where a power $f^{k}, k \geq 1$, of $f$ is isotopic to Dehn twist. In this case we prove that $f$ has a topological horseshoe, if $f$ has at least a fixed point.

Keywords: topological horseshoe, homeomorphism, torus, isotopic to identity, Dehn twist. 


\section{Resumo}

NUNES, P. V. Ferradura topológica para homeomorfismo transitivo do 2-toro. 2021. 81 f. Tese (Doutorado) - Instituto de Matemática e Estatística, Universidade de São Paulo, São Paulo, 2021.

Usando teoria de rotação e teoria de forcing para trajetórias transversas de homeomorfismos de superfície, neste trabalho nós estudamos a relação entre homeomorfismo transitivo do 2-toro, $f: \mathbb{T}^{2} \rightarrow \mathbb{T}^{2}$, e a existência de ferradura topológica.

No caso em que $f$ é isotópico à identidade, nós mostramos que $f$ tem ferradura topológica, se $f$ tem um ponto fixo e um ponto periódico não-fixo.

No caso em que uma potência $f^{k}, k>1$, é isotópica a identidade mas a própria $f$ não é, nós mostramos que se $f$ tem pelo menos um ponto fixo e não tem ferradura topológica então o conjunto de rotação de algum levantamento $\check{f}: \mathbb{R}^{2} \rightarrow \mathbb{R}^{2}$ de $f$, denotado por $\rho(\check{f})$, é somente a origem.

Estudamos também o caso em que uma potência $f^{k}, k \geq 1$, de $f$ é isotópico à Dehn twist. Nesse caso nós mostramos que $f$ tem ferradura topológica, se $f$ tem pelo menos um ponto fixo.

Palavras-chave: ferradura topológica, homeomorfismo, toro, isotópico à identidade, Dehn twist. 


\section{Contents}

List of Figures $\quad$ ix

$\begin{array}{lll}1 & \text { Introduction } & 1\end{array}$

1.1 Motivation and Statement of the Problem . . . . . . . . . . . . . 4

1.2 Research Approach . . . . . . . . . . . . . . . . . . . . . . 7

1.3 Structure of the Thesis . . . . . . . . . . . . . . . . . . . . 8

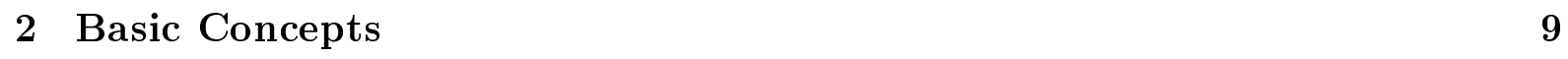

2.1 Covering Space . . . . . . . . . . . . . . . . . . . 11

2.2 Homeomorphisms Isotopic to Identity . . . . . . . . . . . . . . . . . 14

2.2 .1 Isotopy Class of 2-torus homeomorphisms . . . . . . . . . 16

$\begin{array}{lll}3 & \text { Rotation Set Theory } & 19\end{array}$

3.1 2-Torus Homeomorphism . . . . . . . . . . . . . . . . . 19

3.2 Annulus Homeomorphism . . . . . . . . . . . . . . . 23

$\begin{array}{lll}4 & \text { Forcing Theory } & 27\end{array}$

4.1 Singular Oriented Foliations . . . . . . . . . . . . . . 27

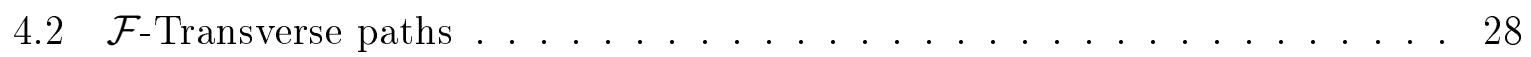

$4.2 .1 \quad \mathcal{F}$-transverse intersection . . . . . . . . . . . . . . . . 30

4.3 Brouwer - Le Calvez Foliation . . . . . . . . . . . . . . . . . . . 34

4.3 .1 Maximal Isotopy and Transverse Foliations . . . . . . . . . . . 36

4.4 Transverse Trajectories . . . . . . . . . . . . . . . . . . . 37

5 Proofs of Theorem A and Proposition C 41

5.1 Proof of Theorem A . . . . . . . . . . . . . . . 48

$5.1 .1 \quad$ Loop homotopic to zero on $\mathbb{T}^{2} \ldots \ldots \ldots \ldots$. . . . . . . 49

$5.1 .2 \quad$ Essential loop on $\mathbb{T}^{2} \ldots \ldots \ldots \ldots \ldots \ldots$

5.2 Proof of Proposition C . . . . . . . . . . . . . . 54

6 Proof of Theorem B $\quad 57$

6.1 If $\rho_{V}(\hat{g})=\{0\} \ldots \ldots \ldots \ldots \ldots \ldots$. . . . . . . . . . . . . . . . .

6.2 If $\rho_{V}(\hat{g})$ is a non-degenerate compact interval of $\mathbb{R}$ containing $\{0\} \ldots 62$ 
viii CONTENTS

Bibliography 


\section{List of Figures}

1.1 Smale Horseshoe. . . . . . . . . . . . . . . . . . . . . . . 1

1.2 Smale Horseshoe with crossing number $M=2 . \ldots \ldots \ldots \ldots$

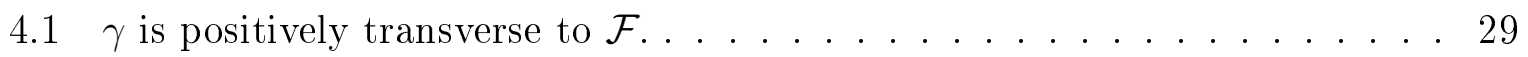

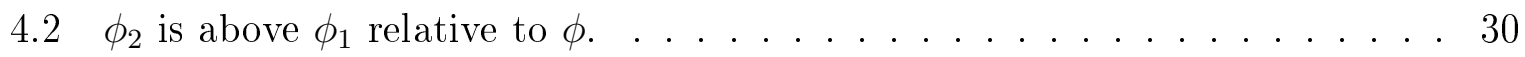

$4.3 \gamma_{1}$ intersects $\mathcal{F}$-transversally $\gamma_{2} . \ldots \ldots \ldots \ldots \ldots \ldots \ldots \ldots$

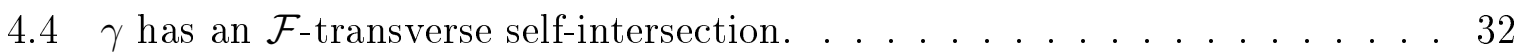

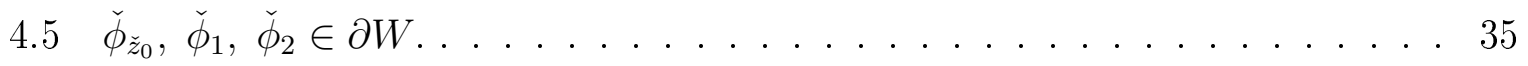

$4.6 \quad \tilde{I}_{\tilde{\mathcal{F}}}^{n}\left(\tilde{z}^{\prime}\right)$ is $\tilde{\mathcal{F}}_{\text {-equivalent }} \tilde{I}_{\tilde{\mathcal{F}}}^{n+2}\left(\tilde{f}^{-1}\left(\tilde{z}^{\prime \prime}\right)\right)$ in $\tilde{\operatorname{dom}}(I) . \ldots \ldots 39$

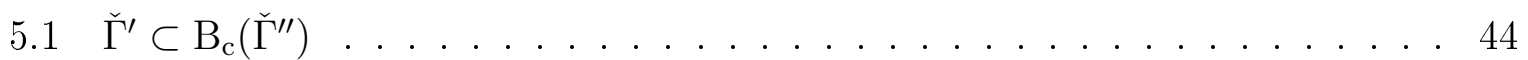

$5.2 \quad J$ and $J^{\prime}$ are crossing components of $J_{\check{\Gamma}_{1}}^{\gamma} \cdot \ldots \ldots \ldots \ldots \ldots \ldots \ldots \ldots$

6.1 Existence of a continuum $\hat{K} . \ldots \ldots \ldots \ldots$. . . . . . . . 6 . 61 
$\mathrm{x}$ LIST OF FIGURES 


\section{Chapter 1}

\section{Introduction}

In Dynamical Systems, an interesting question is understanding ways to measure how "complicated" or "rich" a dynamical system can be, in the sense of how many different behaviors we can observe.

Given $X$ a Hausdorff locally compact topological space and $f$ a homeomorphism of $X$ into itself, Le Calvez and Tal in [LCT18b] define the dynamical system $(X, f)$ as being topologically chaotic if its topological entropy ${ }^{1}$ is positive and if the number of periodic points of period $n$, for some iterate of $f$, grows exponentially in $n$.

An example of such system is the Smale horseshoe, due to the great work of Smale [Sma67]. Basically, it is considered a diffeomorphism $f$ of the plane and a rectangle $\Delta$ that it is linearly contracted vertically, stretched horizontally and then folded so that its image forms a figure similar to a "horseshoe" and such that $\Delta \cap f(\Delta)$ consists of two disjoint "horizontal" rectangles $\Delta_{0}$ and $\Delta_{1}$ (see Figure 1.1).

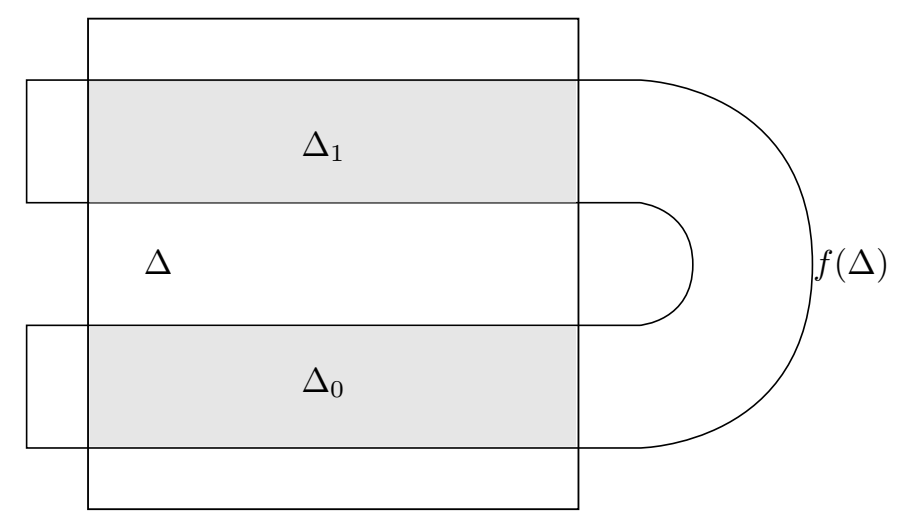

Figure 1.1: Smale Horseshoe.

Smale shows that there exists a compact and $f$-invariant subset $\Delta_{I} \subset \Delta$ such that $\left.f\right|_{\Delta_{I}}$ is topologically conjugate to the Bernoulli shift $\sigma_{2}$ of two symbols.

As topological conjugacy preserves topological properties and the Bernoulli shift is a well-known model with a 'rich' dynamics, we have that $\left.f\right|_{\Delta_{I}}$ inherits such properties.

\footnotetext{
${ }^{1}$ The topological entropy of a homeomorphism $f$, namely $h(f)$, of a Hausdorff locally compact topological space $X$ will be defined as the topological entropy of the extension of $f$ to the Alexandrov one point compactification of $X$, that fixes the point at infinity.
} 
Examples of such properties are topological transitivity, dense periodic orbits, correspondingly the number of periodic points of period $n$ for $\left.f\right|_{\Delta_{I}}$ is $2^{n}$, and positive topological entropy $h(f) \geq h\left(\left.f\right|_{\Delta_{I}}\right)=\log (2)$. And, therefore such dynamic is topologically chaotic.

Since the work of Smale, finding a horseshoe for the dynamics has been a reference feature for saying how rich a dynamical system is. However, most typical constructions of similar objects are based on diffeomorphisms of the plane. To deal with homeomorphisms, or even just continuous map, Kennedy and Yorke in [KY01 formulate an idea for what they called a topological horseshoe, and which we will call here a $K Y$-topological horseshoe.

Their idea of a KY-topological horseshoe for a continuous map $f$ is a compact and connected set $\Delta$ that contains an invariant set $\Delta_{I}$ such the restriction $\left.f\right|_{\Delta_{I}}$ is semiconjugate to a Bernoulli shift $\sigma_{M}$ of $M$ symbols. To construct this semiconjugation, the authors define what they called the "crossing number" $M$ of the set $\Delta$. Let us explain better.

Let $X$ be a separable metric space, $\Delta \subset X$ be a locally connected and compact subset and $f: \Delta \rightarrow X$ be a continuous map. Let $\operatorname{end}_{0}$, $\operatorname{end}_{1} \subset \Delta$ be compact disjoint sets such each component of $\Delta$ intersect both of them. A connection $\Gamma$ is a compact connected subset of $\Delta$ that intersects both end $\mathrm{d}_{0}$ and end $_{1}$. And, a preconnection $\gamma$ is a compact connected subet of $\Delta$ for which $f(\gamma)$ is a connection. In Figure 1.2 , we see that the connection $\Gamma$ has two disjoint preconnection $\gamma_{0}$ and $\gamma_{1}$, in pink and blue, respectively.

The crossing number $M$ is defined as been the largest number such that every connection contains at least $M$ mutually disjoint preconnections.

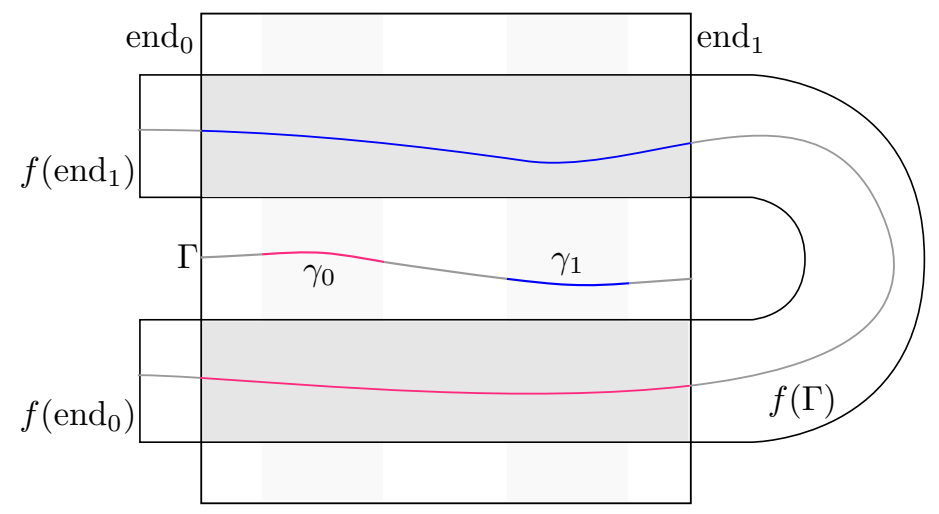

Figure 1.2: Smale Horseshoe with crossing number $M=2$.

Then, Kennedy and Yorke show the following:

Theorem 1.1 (Theorem 1 in [KY01]). Let $X$ and $\Delta \subset X$ be as before. If $f: \Delta \rightarrow X$ is a continuous map and $\Delta$ has crossing number $M \geq 2$ then there is a closed invariant set $\Delta_{I} \subset \Delta$ for which $\left.f\right|_{\Delta_{I}}$ is semiconjugate to a one-sided $M$-shift. In addition, if $f$ is a homeomorphism then $\left.f\right|_{\Delta_{I}}$ is semiconjugate to a two-sided $M$-shift.

Unlike the Smale horseshoe, the semiconjugacy at Theorem 1.1 does not guarantee that the invariant set $\Delta_{I}$ contains periodic points, see the Example 10 in [KY01. But the dynamics remains 'rich', its topological entropy is at least $\log (M)$, indeed $h(f) \geq$ $h\left(\left.f\right|_{\Delta_{I}}\right) \geq h\left(\sigma_{M}\right)=\log (M)$. 
In this work we will define topological horseshoe as it was defined by Le Calvez and Tal in [LCT18b]. Indeed, the definition of Le Calvez and Tal follows the one due to Kennedy and Yorke, but with a few more specifications.

Definition 1.2 (Topological Horseshoe). Let $X$ be a Hausdorff locally compact topological space and $f: X \rightarrow X$ be a homeomorphism. A topological horseshoe for $f$ is a compact subset $\Delta$ of $X$ that is invariant by a positive power $f^{k}$ of $f$ and $\left.f^{k}\right|_{\Delta}$ admits a finite extension ${ }^{2} g: Y \rightarrow Y$ where $Y$ is a Hausdorff compact space and there is an integer $q \geq 2$ such that

1. $g$ is semi-conjugate to the Bernoulli shift of $q$ symbols, $\sigma_{q}: \Sigma \rightarrow \Sigma$, where $\Sigma=$ $\{0, \cdots, q-1\}^{\mathbb{Z}}$

2. The pre-image of every $n$-periodic sequence of $\Sigma$, by the factor map $\Pi_{2}: Y \rightarrow \Sigma$, contains an $n$-periodic point of $g$.

So, we have that the following diagram commutes:

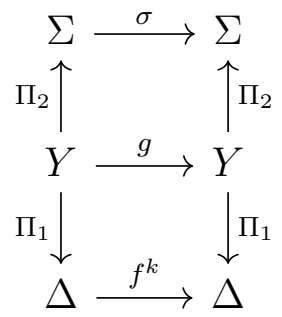

Definition 1.2 tell us that a topological horseshoe for $f$ is a compact subset $\Delta f^{k}$ invariant for which $\left.f^{k}\right|_{\Delta}$ is semiconjugate to $g: Y \rightarrow Y$ with a uniform bound in the cardinality of the fibers of the factor map. And moreover, $Y$ is a $\mathrm{KY}$-topological horseshoe for $g$ with the property that the preimage of every $n$-periodic sequence of $\Sigma$, contains an $n$-periodic point of $g$.

Consequently, if $f$ has a topological horseshoe then $f$ is topologically chaotic. Indeed, we have that $f$ has positive topological entropy:

$$
k h(f)=h\left(f^{k}\right) \geq h\left(\left.f^{k}\right|_{\Delta}\right) \geq h(g) \geq h(\sigma)=\log (q)>0 .
$$

And, as the cardinality of the fibers of the factor map $\Pi_{1}$ is uniformly bounded, saying by a constant $L>0$, we have that $f^{k n}$ has at least $q^{n} / L$ fixed points for every $n \geq 1$.

In the case of surfaces, a classical result involving existence of a horseshoe is due to Katok (see [Kat80]). The author shows that for any $C^{1+\epsilon}$ diffeomorphism of a surface, strictly positive topological entropy implies in the existence of a topological horseshoe. However, this is not true for the case of surfaces homeomorphisms, as we explain in the following.

\footnotetext{
${ }^{2}$ By a finite extension we mean an extension such the fibers of the factor map $\Pi_{1}$ are all finite with a uniform bound in their cardinality.
} 
Given a homeomorphism $f: S \rightarrow S$ defined on a surface $S$, we say that $f$ is topologically transitive if there exists a dense orbit for the map $f$. And if all orbits for $f$ are dense, then the map is called minimal. So, a minimal homeomorphism does not have periodic points, consequently, it does not have topological horseshoe.

Consider the $n$-dimensional torus $\mathbb{T}^{n}$ as the quotient space $\mathbb{R}^{n} / \mathbb{Z}^{n}$. Rees in Ree81 constructed an example of a minimal homeomorphism $f: \mathbb{T}^{n} \rightarrow \mathbb{T}^{n}$ with positive topological entropy. So this example tell us that positive topological entropy does not imply topological horseshoe, for the case of surfaces homeomorphism.

\subsection{Motivation and Statement of the Problem}

From this point on, and until the end of the introduction, we will establish, in general, our problem and our research approach. Consequently, we will omit the definitions of some concepts and terms that we will use here. But they will be defined precisely throughout the text.

The motivation for this work was Theorem $K$ in [LCT18a]. It is as follows:

Theorem 1.3. Let $f: \mathbb{S}^{2} \rightarrow \mathbb{S}^{2}$ be an orientation preserving homeomorphism of the 2dimensional sphere such that the complement of the fixed point set is not an annulus. If $f$ is topologically transitive then the number of periodic points of period $n$ for some iterate of $f$ grows exponentially in $n$. Moreover, the entropy of $f$ is positive.

Indeed, from the proof of this theorem and the techniques later developed in [LCT18b], we can conclude, under those assumptions, that $f$ has a topological horseshoe.

Theorem 1.3 is an improvement of a theorem due to Handel in [Han92]. Indeed, Handel proved that an orientation preserving homeomorphism $f$ of $\mathbb{S}^{2}$ with a dense orbit and whose fixed point set is a finite set containing at least three points, then the number of periodic points of period $n$ for some iterate of $f$ grows exponentially with $n$.

Handel's assumptions are in agreement with Theorem 1.3 because if fix $(f)$ is a finite set containing at least three points then $\mathbb{S}^{2} \backslash \operatorname{fix}(f)$ is not an annulus. So, Handel's assumptions also implies that $f$ has a topological horseshoe.

Motivated by Theorem 1.3, our first question is the following:

Question 1. What are the sufficient conditions so that a topologically transitive homeomorphism of the 2-torus $\mathbb{T}^{2}$ has a topological horseshoe?

The existence of at least one fixed point is necessary to prevent examples like in Ree81. But is it sufficient? The answer is no.

Let Homeo $\left(\mathbb{T}^{2}\right)$ be the space of homeomorphism defined on $\mathbb{T}^{2}$, furnished with the $C^{0}$ topology and $\mathrm{Homeo}_{0}\left(\mathbb{T}^{2}\right)$ its subspace of the homeomorphisms isotopic to identity. In [TAZ07] Tal and Addas-Zanata construct an example, inspired by one which is attributed to Katok (see [FM90]), of an area preserving homeomorphism $h \in \mathrm{Homeo}_{0}\left(\mathbb{T}^{2}\right)$ whose 
rotation set ${ }^{3}$ is a segment with irrational slope, with only one fixed point and no invariant disks.

As $h \in \mathrm{Homeo}_{0}\left(\mathbb{T}^{2}\right)$ is a non-wandering homeomorphism ${ }^{4}$ (because it is area preserving) and its rotation set is a non-degenerate line segment with irrational slope we have, by a result due to Koropecki and Tal (Corollary E in [KT14]), that the absence of invariant disks implies in topological transitivity. However $h$ has no topological horseshoe because by its construction, there is only one periodic point, that is fixed.

Exploring this example, we see that it is possible to build a topologically transitive area preserving homeomorphism of $\mathbb{T}^{2}$ isotopic to identity with finitely many periodic points (with the same period) without a topological horseshoe.

Our first theorem shows that in some sense, a dynamical system in the 2-torus without topological horseshoes cannot be much more complicated than this example, at least in the isotopy class of identity.

Theorem A. Let $f \in \mathrm{Homeo}_{0}\left(\mathbb{T}^{2}\right)$ be topologically transitive. If $f$ has a fixed point and a non-fixed periodic point then $f$ has a topological horseshoe.

Theorem A is established for homeomorphisms in the isotopy class of identity. But it is already known that to classify $f \in \operatorname{Homeo}\left(\mathbb{T}^{2}\right)$ up to isotopy amounts to classifying the linear automorphisms of $\mathbb{T}^{2}$. Indeed, given $f \in \operatorname{Homeo}\left(\mathbb{T}^{2}\right)$ then $f$ is isotopic to a linear automorphism $f_{A} \in \operatorname{Homeo}\left(\mathbb{T}^{2}\right)$ that, in turn, it is one of the following types (for more details, see Chapter 2):

I. Linear Anosov;

II. Periodic and, in this case, there exist some integer $k \geq 1$ such that $f^{k}$ is isotopic to identity;

III. Reducible, but not periodic. So, in this case, there exist some integer $k \geq 1$ such that $f^{k}$ is isotopic to a Dehn Twist.

Given this classification and Theorem A, we can ask ourselves:

Question 2. If $f \in \operatorname{Homeo}\left(\mathbb{T}^{2}\right)$ is topologically transitive, with a non-empty fixed point set and no topological horseshoe then what can we say about the homeomorphism $f$ ? Does $f$ have to be isotopic to identity?

To answer this question we must to analyze the isotopy class that $f$ belongs.

We start by analyzing when $f$ is isotopic to a linear Anosov automorphism. It is already known that the dynamics of a linear Anosov automorphism of $\mathbb{T}^{2}$ is very 'rich' (see [KH97], for example). Moreover, if $f$ is isotopic to a linear Anosov automorphism, then the dynamics of $f$ is "at least as rich as" $f_{A}$ :

\footnotetext{
${ }^{3}$ See Chapter 3

${ }^{4}$ For a definition see page 10
} 
Theorem 1.4 (Theorem 2.6.1 in [KH97]). Let $f_{A}: \mathbb{T}^{2} \rightarrow \mathbb{T}^{2}$ be a linear Anosov automorphism of $\mathbb{T}^{2}$ and let $f \in \operatorname{Homeo}\left(\mathbb{T}^{2}\right)$ be isotopic to $f_{A}$. Then there exists a continuous surjective application $\Pi: \mathbb{T}^{2} \rightarrow \mathbb{T}^{2}$ such that $\Pi \circ f=f_{A} \circ \Pi$.

In addition, a linear Anosov automorphism has the minimum number of periodic points for any given period among all homeomorphisms of its isotopy class (see [BLR20]).

Proposition 1.5. Let $f \in \operatorname{Homeo}\left(\mathbb{T}^{2}\right)$ be isotopic to a linear Anosov $f_{A} \in \operatorname{Homeo}\left(\mathbb{T}^{2}\right)$. Then for each $n \in \mathbb{N}$ the homeomorphism $f$ has at least as many periodic orbit of period $n$ as $f_{A}$.

Thus, if $f \in \operatorname{Homeo}\left(\mathbb{T}^{2}\right)$ is isotopic to a linear Anosov, then $f$ is topologically chaotic. And, therefore, answering Question 2, if $f \in \operatorname{Homeo}\left(\mathbb{T}^{2}\right)$ has no topological horseshoe, we have that $f$ cannot be isotopic to a linear Anosov.

For the case when some power $f^{k}$ of $f$ is isotopic to a Dehn twist the answer of Question 2 follows from our next theorem:

Theorem B. Let $f \in \operatorname{Homeo}\left(\mathbb{T}^{2}\right)$ be a topologically transitive homeomorphism with a non-empty fixed point set and such that a power $f^{k}$ of $f$, where $k \geq 1$, is isotopic to a Dehn Twist. Then $f$ has a topological horseshoe.

So, Theorem B give an answer to Question 2 saying that, under those assumptions, there is no power $f^{k}$ of $f$ that is isotopic to a Dehn twist.

We still do not have a conclusion for the case where some power $f^{k}$ of $f$, where $k>1$, is isotopic to identity, but $f$ itself is not. In this direction, we get the following proposition:

Proposition C. Let $f \in \operatorname{Homeo}\left(\mathbb{T}^{2}\right)$ be a topologically transitive homeomorphism with a non-empty fixed point set and such that some power $g=f^{k}$ of $f$, where $k>1$, is isotopic to identity, but $f$ itself is not. If $f$ does not have a topological horseshoe, then for some lift $\check{g} \in \operatorname{Homeo}\left(\mathbb{R}^{2}\right)$ of $g$, the rotation set of $\check{g}$ is equal to $\{(0,0)\}$.

And we conjecture the following:

Conjecture D. If $f \in \operatorname{Homeo}\left(\mathbb{T}^{2}\right)$ is a topologically transitive homeomorphism with non-empty fixed point set such that some power $f^{k}$ of $f$, where $k>1$, is isotopic to identity, but $f$ itself is not, then $f$ has topological horseshoe.

If the above conjecture is true, then we can give an affirmative answer to the second question of Question 2, that is:

Conjecture E. Let $f \in \operatorname{Homeo}\left(\mathbb{T}^{2}\right)$ be topologically transitive and such that $\operatorname{fix}(f) \neq \emptyset$. If $f$ does not have a topological horseshoe then $f$ is isotopic to identity.

Another question, which we believe that is a natural continuation of this work is: 
Question 3. If instead of asking that the 2-torus homeomorphism is topologically transitive, we ask that it is non-wandering? What would the absence of topological horseshoe imply about the dynamics?

We do not have an answer to the above question. But, in addition to the ideas presented in this text, another motivation for it is a main decomposition result for a structure theorem for area preserving diffeomorphisms of $\mathbb{S}^{2}$ with zero entropy due to Franks and Handel (see Theorem 1.2 in [FH13]), which was extended to non-wandering homeomorphism case by Le Calvez and Tal (see Theorem M in [LCT18a]).

\subsection{Research Approach}

Our proofs to the above statements are based mainly on forcing theory for transverse trajectories of surface homeomorphisms developed by Le Calvez and Tal in [LCT18a] and [LCT18b], and also on rotation set theory.

Rotation set theory started with Poincaré (see [Poi52]), who introduced the concept of rotation number of a homeomorphism of the circle, $\mathbb{T}^{1}=\mathbb{R} / \mathbb{Z}$ that preserves orientation. Poincaré showed that all orbits have the same rotation number and, moreover, that this number is a topological invariant.

Indeed, if it is a rational number then there always exist periodic orbits and all of them have the same period. Besides, all non-periodic orbits are asymptotic to periodic orbits. If the rotation number is an irrational number, then there are no periodic orbits: either all orbits are dense or all orbits are asymptotic to a Cantor set (Denjoy's Example).

The concept of rotation number was generalized to the case of 2-torus homeomorphisms that are isotopic to identity by Misiurewicz and Ziemian as a rotation set, and no longer as a rotation number, see [MZ89.

In rotation theory, an important area of interest is the study of the relation between the rotation set and the behavior of the orbits. Although not as simple as for circle homeomorphisms, several authors, following Misiurewicz and Ziemian, have shown interesting results in this regard: [Fra88], [Fra89], [LM91], [KK08], [Dáv18], [LCT18a], [LCT18b], and several others. We will review some of those works in Chapter 3 .

Misiurewicz and Ziemian in [MZ89] showed that the rotation set of a 2-torus homeomorphism isotopic to identity is non-empty, compact and convex set of $\mathbb{R}^{2}$.

The case in which the rotation set has non-empty interior is well understood. Llibre and MacKay [LM91] showed that in this case the dynamics has positive topological entropy, in fact it has a topological horseshoe. Regarding periodic points, Franks in [Fra89] showed that if the rotation set has a non-empty interior, every vector of rational coordinates in the interior of the rotation set is realized by periodic points. This means that given $f \in \operatorname{Homeo}_{0}\left(\mathbb{T}^{2}\right)$ and a lift $\check{f} \in \operatorname{Homeo}\left(\mathbb{R}^{2}\right)$ of $f$, for every $\left(\frac{p_{1}}{q}, \frac{p_{2}}{q}\right) \in \mathbb{Q}^{2}$ in the interior of the rotation set, there is a point $\check{z} \in \mathbb{R}^{2}$ such that $\check{f}^{q}(\check{z})=\check{z}+\left(p_{1}, p_{2}\right)$. 
But when the rotation set has an empty interior, the scenario is different. There are simple examples showing that we can have homeomorphisms whose rotation set is a segment with infinitely many rational points, but with no periodic points. However, Franks in [Fra95] and, later, Kocsard and Koropecki in [KK08] presented sufficient conditions under which, if the rotation set is a line segment, every rational vector is realized by a periodic orbit.

Dávalos in [Dáv18] characterized the homeomorphisms of the 2-torus isotopic to identity under which the rotation set is a line segment with rational slope. The author showed that, in this situation, there exist uniformly bounded deviation in the direction perpendicular to the rotation set of some lift for some power of $f$.

Together with rotation theory, forcing theory for transverse trajectories of surface homeomorphisms underlies and supports this work. The basis of this theory is contained in both articles [LCT18a] and [LCT18b] due to Le Calvez and Tal.

Forcing Theory is an improvement of Brouwer's Theory and is a purely topological technique that has been shown a great tool in the study of surface homeomorphisms. For its development, a self-homeomorphism $f$ isotopic to identity defined on an oriented surface is considered.

The idea is to explore the Equivariant Foliation Theorem, due to Le Calvez (see [LC05], Theorem 1.2) that together with a recent result due to Béguin, Crovisier and Le Roux about the existence of a maximal isotopy (see [BCLR20]) allow us to build a singular oriented foliation $\mathcal{F}$ on the surface that is topologically transverse to this maximal isotopy, see Subsection 4.3.1. And studying the dynamics of the foliation that is transverse to the maximal isotopy we can obtain new properties of $f$, for example, deduce the existence of new orbits and even if it is possible to have a topological horseshoe for the dynamics, see Proposition 4.26 and Theorem 4.28 .

\subsection{Structure of the Thesis}

In Chapter 2 we will present a collection of introductory definitions and basic concepts that will be useful in the whole text.

In Chapters 3 and 4 we will present and give more details about Rotation Set Theory and Forcing Theory for transverse trajectories of surface homeomorphisms, respectively.

Chapters 5 and 6 are reserved for the proofs of the previously proposed statements. Theorem A and Proposition C are in Chapter 5 , while Theorem B is in Chapter 6. 


\section{Chapter 2}

\section{Basic Concepts}

In this chapter we will give some introductory definitions and basic concepts about topological dynamics of surfaces that will be useful in the whole text. In Subsection 2.2.1 we will present the isotopy classes and linear automorphisms of 2-torus homeomorphisms. For more details the reader can see [KH97], [Hat02] and [BLR20].

We will endow $\mathbb{R}^{2}$ with its usual orientation and with the Euclidean scalar product $\langle\cdot, \cdot\rangle$. We will write $\|\cdot\|$ and $\mathrm{d}(\cdot, \cdot)$ for the associated norm and metric. We will consider the $n$-dimensional torus $\mathbb{T}^{n}$ as the quotient space $\mathbb{R}^{n} / \mathbb{Z}^{n}$ and we will denote by $\mathbb{A}=\mathbb{T}^{1} \times \mathbb{R}$ the (vertical) annulus. Also, we will denote the 2-dimensional sphere by $\mathbb{S}^{2}$.

Let $S$ be an oriented surface, that is a Hausdorff topological space locally homeomorphic to $\mathbb{R}^{2}$. A subset $X$ of a surface $S$ is called an open disk if it is homeomorphic to $D=\left\{z \in \mathbb{R}^{2},\|z\|<1 \mid\right\}$, a closed disk if it is homeomorphic to $\bar{D}=\left\{z \in \mathbb{R}^{2},\|z\| \leq 1\right\}$, and it is called an annulus if it is homeomorphic to $\mathbb{T}^{1} \times J$, where $J$ is a non-trivial interval of $\mathbb{R}$. If $J=[0,1]$ or $J=(0,1)$ we have a closed and an open annulus, respectively.

Let $\operatorname{Homeo}(S)$ be the space of homeomorphism defined on $S$, furnished with the $C^{0}$ topology. Given $f \in \operatorname{Homeo}(S)$ and $z \in S$, the $\alpha$-limit and $\omega$-limit sets of $z$ are, respectively,

$$
\begin{aligned}
& \alpha_{f}(z)=\left\{w \in S \mid \exists\left(n_{i}\right)_{i \in \mathbb{N}} \subset \mathbb{N} \text { such that } n_{i} \nearrow+\infty \text { and } f^{-n_{i}}(z) \stackrel{i \rightarrow+\infty}{\longrightarrow} w\right\} \\
& \omega_{f}(z)=\left\{w \in S \mid \exists\left(n_{i}\right)_{i \in \mathbb{N}} \subset \mathbb{N} \text { such that } n_{i} \nearrow+\infty \text { and } f^{n_{i}}(z) \stackrel{i \rightarrow+\infty}{\longrightarrow} w\right\} .
\end{aligned}
$$

We say that a point $z \in S$ is positively or negatively recurrent if $z \in \omega_{f}(z)$ or $z \in \alpha_{f}(z)$, respectively, and bi-recurrent if it is both positively and negatively recurrent.

Lemma 2.1. Let $f \in \operatorname{Homeo}(S)$. Take any integer $k \geq 1$ and let $g=f^{k}$. If there is a point $z \in \mathbb{T}^{2}$ such that $\omega_{f}(z)=S$ then

$$
\bigcup_{r=0}^{k-1} \omega_{g}\left(f^{r}(z)\right)=S .
$$

Proof. Let $w \in S$ be any point. As $\omega_{f}(z)=S$ then $w \in \omega_{f}(z)$, and by the definition of 
$\omega$-limit set, there is an increasing sequence $\left(n_{i}\right)_{i \in \mathbb{N}}$ of positive integers such that $n_{i} \rightarrow+\infty$ and

$$
\lim _{i \rightarrow+\infty} f^{n_{i}}(z)=w .
$$

Now, as $n_{i} \in \mathbb{N}$ for all $i \in \mathbb{N}$, there exist $m_{i} \in \mathbb{N}$ and $r_{i} \in\{0,1, \cdots, k-1\}$ such that $n_{i}=m_{i} k+r_{i}$. Then, we have

$$
f^{n_{i}}(z)=f^{m_{i} k+r_{i}}(z)=g^{m_{i}}\left(f^{r_{i}}(z)\right) \text {, for each } i \in \mathbb{N} .
$$

But, as $\left(r_{i}\right)_{i \in \mathbb{N}}$ can only assume finite values, we have, by the Pigeonhole Principle, that there is a subsequence $\left(r_{i_{j}}\right)_{j \in \mathbb{N}}$ of $\left(r_{i}\right)_{i \in \mathbb{N}}$ and $r \in\{0,1, \cdots, k-1\}$ such that $r_{i_{j}}=r$ for all $j \in \mathbb{N}$. Therefore, if we consider the subsequence

$$
\left(g^{m_{i_{j}}}\left(f^{r_{i_{j}}}(z)\right)\right)_{j \in \mathbb{N}}=\left(g^{m_{i_{j}}}\left(f^{r}(z)\right)\right)_{j \in \mathbb{N}} \subset\left(g^{m_{i}}\left(f^{r_{i}}(z)\right)\right)_{i \in \mathbb{N}},
$$

we have

$$
\lim _{j \rightarrow+\infty} g^{m_{i_{j}}}\left(f^{r}(z)\right)=\lim _{i \rightarrow+\infty} g^{m_{i}}\left(f^{r_{i}}(z)\right)=\lim _{i \rightarrow+\infty} f^{n_{i}}(z)=w,
$$

because a subsequence of a convergent sequence also converges to the same limit of it.

This implies that there exists some $r \in\{0,1, \cdots, k-1\}$ such that $w \in \omega_{g}\left(f^{r}(z)\right)$, or equivalently, $w \in \bigcup_{r=0}^{k-1} \omega_{g}\left(f^{r}(z)\right)$ as we wanted.

We can also establish an analogous result for the $\alpha$-limit set.

Lemma 2.2. Let $f \in \operatorname{Homeo}(S)$. Take any integer $k \geq 1$ and let $g=f^{k}$. If there is a point $z \in \mathbb{T}^{2}$ such that $\alpha_{f}(z)=S$ then

$$
\bigcup_{r=0}^{k-1} \alpha_{g}\left(f^{-r}(z)\right)=S .
$$

Proof. Analogous to the proof of Lemma 2.1.

Remember that at the introduction we defined a topologically transitive map as being the one that has a dense orbit. In particular, it is possible to show that if the surface $S$ is compact, then $f \in \operatorname{Homeo}(S)$ is topologically transitive if and only if there is some $z \in S$ such that $\omega_{f}(z)=\alpha_{f}(z)=S$.

Given $f \in \operatorname{Homeo}(S)$, we say that a point $z \in S$ is non-wandering for $f$ if any neighborhood $U \subset S$ of $z$ is such that $f^{n}(U) \cap U \neq \emptyset$ for some $n \in \mathbb{N}$. Otherwise, the point $z$ is said wandering for $f$. We will represent the set of the non-wandering points for $f$ by $\Omega(f)$. Moreover, we say that $f \in \operatorname{Homeo}(S)$ is a non-wandering homeomorphism if every point $z \in S$ is non-wandering for $f$, that is $\Omega(f)=S$.

The following definition is about Birkhoff recurrence classes.

Definition 2.3. Let $X$ be a metric space, $f \in \operatorname{Homeo}(X)$, and $z_{1}, z_{2}$ two points of $X$. 
1. We say that there exists a Birkhoff connection from $z_{1}$ to $z_{2}$ if for every neighborhood $W_{1}$ of $z_{1}$ and every neighborhood $W_{2}$ of $z_{2}$, there is $n>1$ such that $W_{1} \cap f^{-n}\left(W_{2}\right) \neq$ $\emptyset$.

2. A Birkhoff cycle is a finite sequence $\left(z_{i}\right)_{i \in \mathbb{Z} / p \mathbb{Z}}$ for every $i \in \mathbb{Z} / p \mathbb{Z}$ there is a Birkhoff connection from $z_{i}$ to $z_{i+1}$.

3. A point $z$ is said to be Birkhoff recurrent for $f$ if there is a Birkhoff cycle containing $z$.

4. We can define an equivalence relation in the set of Birkhoff recurrent points: say that $z_{1}$ is Birkhoff equivalent to $z_{2}$ if there exists a Birkhoff cycle containing both points. The equivalence classes will be called Birkhoff recurrence classes.

Note that every $\omega$-limit set or $\alpha$-limit set is contained in a single Birkhoff recurrence class. Indeed, let $z_{1}, z_{2} \in \omega_{f}(z)$ and let $W_{1}, W_{2} \subset X$ be neighborhood of $z_{1}$ and $z_{2}$ respectively. So, there are an increasing sequences $n_{k}, m_{l} \in \mathbb{N}$ going to $+\infty$ such that $f^{n_{k}}(z) \rightarrow z_{1}$ and $f^{m_{l}}(z) \rightarrow z_{2}$. This implies that there is some $k>1$ large enough such that $f^{n_{k}}(z) \in W_{1}$ and there is some $l>1$ large enough such that $m_{l}>n_{k}$ and $f^{m_{l}}(z) \in W_{2}$. So, $W_{1} \cap f^{-n}\left(W_{2}\right) \neq \emptyset$, where $n=m_{l}-n_{k}$. Therefore, there is a Birkhoff connection from $z_{1}$ and $z_{2}$. But this is true for any two points in $\omega_{f}(z)$, thus there is a Birkhoff connection from $z_{2}$ and $z_{1}$. Therefore, any two such points are in the same Birkhoff recurrence class.

\subsection{Covering Space}

A method to study the dynamics of a surface homeomorphism $f$ is to consider a lift $\breve{f}$ of $f$ to the universal covering space of the surface. Let us explain better.

Let $S$ be a surface. A map $\breve{\pi}: \breve{S} \rightarrow S$ is called covering projection if it satisfies the following property: any point $z \in S$ admits a neighborhood $U \subset S$ such that $\breve{\pi}^{-1}(U)=\bigcup_{i} \breve{U}_{i}$ is a disjoint union of open sets $\breve{U}_{i}$ and each restriction $\left.\breve{\pi}\right|_{\breve{U}_{i}}: \breve{U}_{i} \rightarrow U_{i}$ is a homeomorphism. The space $\breve{S}$ is called a covering space of $S$.

It is possible to show that a surface $S$ admits a covering projection $\breve{\pi}: \breve{S} \rightarrow S$ where $\breve{S}$ is simply connected. In particular, if $\breve{S}$ is a simply connected space the covering projection $\breve{\pi}: \breve{S} \rightarrow S$ is called universal covering projection, because $\breve{S}$ covers any other covering space $\breve{S}^{\prime}$ of $S$.

We have that $\mathbb{R}^{2}$ is the universal covering space of $\mathbb{T}^{2}$ and $\mathbb{A}$, moreover $\mathbb{A}$ is also a covering space of $\mathbb{T}^{2}$. So we will establish the following notations:

Notation 2.4. Let $\mathbb{R}^{2}, \mathbb{T}^{2}$ and $\mathbb{A}$ as before, then: 
1. A point in $\mathbb{R}^{2}$ will be denoted with a check mark: $\check{z}=(\check{x}, \check{y}) \in \mathbb{R}^{2}$. A point in $\mathbb{A}$ will be denoted with a hat mark: $\hat{z}=(\hat{x}, \hat{y}) \in \mathbb{A}$. And, finally, a point in $\mathbb{T}^{2}$ will be denoted without any mark: $z=(x, y) \in \mathbb{T}^{2}$;

2. $\mathrm{p}_{1}: \mathbb{R}^{2} \rightarrow \mathbb{R}$ and $\mathrm{p}_{2}: \mathbb{R}^{2} \rightarrow \mathbb{R}$ denote the two canonical projections: $\mathrm{p}_{1}(\check{x}, \check{y})=\check{x}$ and $\mathrm{p}_{2}(\check{x}, \check{y})=\check{y}$. And we will also use $\mathrm{p}_{2}$ to denote the second coordinate (vertical) projection in $\mathbb{A}$;

3. $\check{\pi}: \mathbb{R}^{2} \rightarrow \mathbb{T}^{2}$ denotes the canonical universal covering projection of the 2 -torus:

$$
\check{\pi}((\check{x}, \check{y}))=(\check{x}+\mathbb{Z}, \check{y}+\mathbb{Z}) .
$$

4. $\check{\tau}: \mathbb{R}^{2} \rightarrow \mathbb{A}$ denotes the canonical universal covering projection of the annulus:

$$
\check{\tau}((\check{x}, \check{y}))=(\check{x}+\mathbb{Z}, \check{y}) .
$$

5. $\hat{\pi}: \mathbb{A} \rightarrow \mathbb{T}^{2}$ denotes the covering projection from the annulus to the 2 -torus, such that

$$
\check{\pi}=\hat{\pi} \circ \check{\tau}
$$

So, the following diagram commutes:

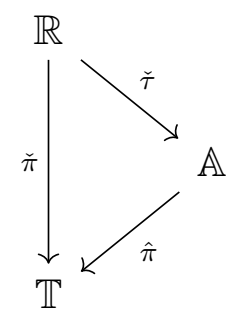

6. A self-homeomorphism of $\mathbb{R}^{2}$ will be denoted with a check mark: $\check{f} \in \operatorname{Homeo}\left(\mathbb{R}^{2}\right)$. For self-homeomorphisms of $\mathbb{A}$ we will denote them with a hat mark: $\hat{f} \in \operatorname{Homeo}(\mathbb{A})$. And in turn, a self-homeomorphism of $\mathbb{T}^{2}$ will be denoted without any mark: $f \in$ $\operatorname{Homeo}\left(\mathbb{T}^{2}\right)$.

We will endow $\mathbb{T}^{2}$ and $\mathbb{A}$ with the following metric:

$$
\begin{aligned}
& d_{\mathbb{T}^{2}}\left(z^{\prime}, z\right)=\inf \left\{d\left(\check{z}^{\prime}, \check{z}\right) \mid \check{z}^{\prime} \in \check{\pi}^{-1}\left(z^{\prime}\right), \check{z} \in \check{\pi}^{-1}(z)\right\}, \text { and } \\
& d_{\mathbb{A}}\left(\hat{z}^{\prime}, \hat{z}\right)=\inf \left\{d\left(\check{z}^{\prime}, \check{z}\right) \mid \check{z}^{\prime} \in \check{\tau}^{-1}\left(\hat{z}^{\prime}\right), \check{z} \in \check{\tau}^{-1}(\hat{z})\right\}, \text { respectively. }
\end{aligned}
$$

Now, we will define a lift $\breve{f}$ of a homeomorphism $f \in \operatorname{Homeo}(S)$ to $\breve{S}$. Let $\breve{\pi}: \breve{S} \rightarrow S$ and $\breve{S}$ be a covering projection and a covering space of the surface $S$, respectively. 
Definition 2.5. Given $f \in \operatorname{Homeo}(S)$ and $\breve{f} \in \operatorname{Homeo}(\breve{S})$ we say that $\breve{f}$ is a lift of $f$ to $\breve{S}$ if

$$
f \circ \breve{\pi}=\breve{\pi} \circ \breve{f}
$$

That is, the following diagram commutes:

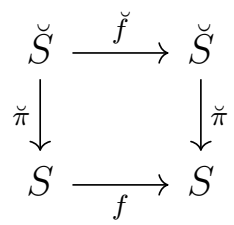

Remark 2.6. Note that given $f \in \operatorname{Homeo}(S)$ and a lift $\breve{f} \in \operatorname{Homeo}(\breve{S})$ of $f$, if $\breve{f}$ has a topological horseshoe, then it imply that $f$ also has a topological horseshoe, as in Definition 1.2 .

In fact, if $\breve{f}$ has a topological horseshoe then there is a compact subset $\breve{\Delta} \subset \breve{S}$ invariant by a power $\breve{f}^{l}$ of $\breve{f}$ and $\left.\breve{f}^{l}\right|_{\breve{\Delta}}$ admits a finite extension $g: Y \rightarrow Y$ on a Hausdorff compact space such that:

1. $g$ is semi-conjugate to the Bernoulli shift of $q$ symbols, $\sigma_{q}: \Sigma \rightarrow \Sigma$, where $\Sigma=$ $\{0, \cdots, q-1\}^{\mathbb{Z}}$

2. The pre-image of every $n$-periodic sequence of $\Sigma$, by the factor map $\Pi_{2}: Y \rightarrow \Sigma$, contains an $n$-periodic point of $g$.

So, the covering projection $\breve{\pi}: \breve{S} \rightarrow S$, restrict to $\breve{\Delta}$, induces a semi-conjugacy between $\left.\breve{f}^{l}\right|_{\breve{\Delta}}$ and $\left.f^{l}\right|_{\breve{\pi}(\breve{\Delta})}$. And as the set $\breve{\Delta}$ is compact, its projection is a compact subset $\Delta=\breve{\pi}(\breve{\Delta})$ on $S$ which is $f^{l}$-invariant. Furthermore every point in $\Delta$ has finitely many lifts on $\breve{\Delta}$ with a uniform upper bound. Then there is the following semi-conjugation

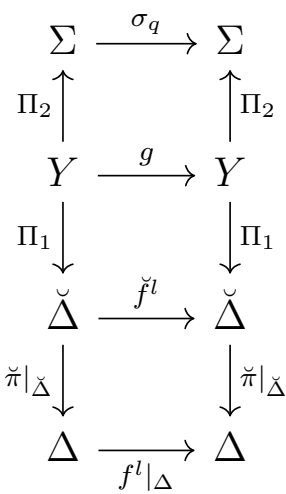

where $g$ is a finite extension for $\left.f^{l}\right|_{\Delta}$ that is semi-conjugated to the Bernoulli shift and has the property that the preimage of every $n$-periodic sequence of $\{0, \cdots, q-1\}^{\mathbb{Z}}$ by the factor map $\Pi_{2}: Y \rightarrow\{0, \cdots, q-1\}^{\mathbb{Z}}$ contains an $n$-periodic point of $f$. Therefore $\Delta$ is a topological horseshoe for $f$ on $S$.

Now, let $\breve{S}$ be the universal covering of $S$, that is the covering space that is simply connected, and $\breve{\pi}: \breve{S} \rightarrow S$ be a universal covering projection. 
A homeomorphism $T: \breve{S} \rightarrow \breve{S}$ such that

$$
\breve{\pi} \circ T=\breve{\pi}
$$

is called a covering automorphism of $S$. Note that a covering automorphism is a lift of the identity of $S$ to its universal covering space $\breve{S}$.

The set of covering automorphism of the surface $S$ will be denoted by $\operatorname{Deck}(S)$. It is possible to show that $\operatorname{Deck}(S)$ forms a discrete group under composition, that acts freely and properly on $\breve{S}$, see definition below.

Definition 2.7. Let $G$ be a discrete group of homeomorphisms of $\breve{S}$ that preserve orientation. We say that

1. G acts freely on $\breve{S}$ if given any $g \in G$ that there exists some $\breve{z} \in \breve{S}$ such that $g(\breve{z})=\breve{z}$, then $g=I d$;

2. G acts properly on $\breve{S}$, if for all compact subsets $\breve{K} \subset \breve{S}$, we have $g(\breve{K}) \cap \breve{K} \neq \emptyset$ for only finite homeomorphisms $g \in G$.

For more details of covering automorphisms, see Section 1.3 in Hat02].

In particular, if $S=\mathbb{T}^{2}$, as $\mathbb{R}^{2}$ is the universal covering space of $\mathbb{T}^{2}$ and $\mathbb{A}$, we have, for the universal covering projections fixed on Notation 2.4, that the covering automorphisms of $\mathbb{T}^{2}$ are the translations of $\mathbb{R}^{2}$ by an integer vector, that is,

$$
\operatorname{Deck}\left(\mathbb{T}^{2}\right)=\left\{T: \mathbb{R}^{2} \rightarrow \mathbb{R}^{2} \mid \exists p \in \mathbb{Z}^{2} \text { such that } T: \check{z} \mapsto \check{z}+p\right\}
$$

and the covering automorphisms of $\mathbb{A}$ are the translations of $\mathbb{R}^{2}$ in the first coordinate by an integer number, that is

$$
\operatorname{Deck}(\mathbb{A})=\left\{T: \mathbb{R}^{2} \rightarrow \mathbb{R}^{2} \mid \exists p \in \mathbb{Z} \text { such that } T:(\check{x}, \check{y}) \mapsto(\check{x}+p, \check{y})\right\}
$$

\subsection{Homeomorphisms Isotopic to Identity}

Let $S$ be an oriented surface. Given two homeomorphisms $f, g \in \operatorname{Homeo}(S)$, we say that $f$ is homotopic to $g$ if there is a continuous map $H:[0,1] \times S \rightarrow S$ such that

1. For every $t \in[0,1], I(t, \cdot):=f_{t}: S \rightarrow S$ is a continuous map;

2. $I(0, z)=g(z)$, for all $z \in S$;

3. $I(1, z)=f(z)$, for all $z \in S$.

If there is a homotopy $H$ between $f$ and $g$ such that $H(t, \cdot):=f_{t}: S \rightarrow S$ is a homeomorphism, for every $t \in[0,1]$, then we say that $f$ is isotopic to $g$, and in this case we denote $H$ by $I:[0,1] \times S \rightarrow S$. 
An important result regarding homeomorphisms of the 2-torus that are homotopic is due to Epstein (see [Eps66]):

Theorem 2.8. Given $f, g \in \operatorname{Homeo}\left(\mathbb{T}^{2}\right)$. If $f$ is homotopic to $g$ then $f$ is isotopic to $g$.

We will denote by Id : $S \rightarrow S$ the identity map of $S$ and by $\operatorname{Homeo}_{0}(S)$ the subspace of Homeo $(S)$ of the homeomorphisms that are isotopic to identity.

Let $\mathcal{I}_{f}$ be the space of identity isotopies of $f \in \mathrm{Homeo}_{0}(f)$, that is the set of continuous paths defined on $[0,1]$ joining the identity to $f$ in the space $\operatorname{Homeo}(S)$, furnished with the $C^{0}$ topology.

A path on $S$ is a continuous map $\gamma: J \rightarrow S$ defined on an interval $J \subset \mathbb{R}$. If $I=\left(f_{t}\right)_{t \in[0,1]} \in \mathcal{I}_{f}$, we define the trajectory of the point $z \in S$ along $I$ as the path

$$
I(z): t \mapsto I(z)(t)=f_{t}(z)
$$

Note that the trajectory of $z$ along $I$ is a path joining $z$ to $f(z)$. In general, we can define by concatenation $I^{n}(z)=\prod_{0 \leq k<n} I\left(f^{k}(z)\right)$ for every integer $n \geq 1$. Furthermore, we can define

$$
I^{\mathbb{N}}(z)=\prod_{k \geq 0} I\left(f^{k}(z)\right), I^{-\mathbb{N}}(z)=\prod_{k \leq 0} I\left(f^{k}(z)\right), I^{\mathbb{Z}}(x)=\prod_{k \in \mathbb{Z}} I\left(f^{k}(z)\right),
$$

as the future, past and whole trajectory of a point $z \in S$ along $I$, respectively.

Let $\breve{S}$ be the universal covering space of $S$ and $\breve{\pi}: \breve{S} \rightarrow S$ be a universal covering projection.

Given an identity isotopy $I=\left(f_{t}\right)_{t \in[0,1]} \in \mathcal{I}_{f}$ of $f \in \operatorname{Homeo}_{0}(S)$, we can lift $I$ for an isotopy $\breve{I}=\left(\breve{f}_{t}\right)_{t \in[0,1]}$ such that $\breve{f}_{0}=\left.\operatorname{Id}\right|_{\breve{S}}$ and for all $t \in[0,1], \breve{f}_{t}$ is a lift of $f_{t}$. This isotopy is unique and the homeomorphism $\breve{f}:=\breve{f}_{1}$ is called the lift of $f$ associated to $I$. For more details see Section 1.3 in Hat02. Moreover, in this case we have that $\breve{f}$ commutes with the $\operatorname{Deck}(S)$ transformations:

$$
\breve{f} \circ T=T \circ \breve{f}, \forall T \in \operatorname{Deck}(S)
$$

In particular, if $S=\mathbb{T}^{2}, f \in \operatorname{Homeo}_{0}\left(\mathbb{T}^{2}\right)$ and $\check{f} \in \operatorname{Homeo}\left(\mathbb{R}^{2}\right)$ a lift of $f$ to $\mathbb{R}^{2}$, we have that $\check{f}$ induces a lift $\hat{f} \in \operatorname{Homeo}(\mathbb{A})$ of $f$ to $\mathbb{A}$ such that the following diagram commutes:

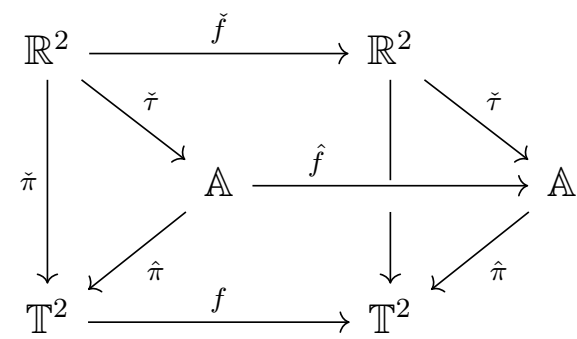




\subsubsection{Isotopy Class of 2-torus homeomorphisms}

The reference for this subsection is BLR20.

Let $f \in \operatorname{Homeo}(S)$ be a homeomorphism of a closed surface $S$. Then $f$ naturally acts on the homology classes of closed curves $\gamma$ of $S$ by

$$
f_{*}:[\gamma] \mapsto[f \circ \gamma]
$$

It is possible to show that the map $f_{*}$, induced by $f$, is an isomorphism and, moreover, that it can be represented by an element of $\operatorname{GL}(2 \mathrm{~g}, \mathbb{Z})$, where $g$ is the genus of the surface $S$ and $\mathrm{GL}(2 \mathrm{~g}, \mathbb{Z})$ is the group of $2 g \times 2 g$ invertible matrices with integer coefficients, thus their determinant is \pm 1 .

Furthermore, we have that if $f$ is homotopic to $g$ then they induce the same action on the closed curves, that is $f_{*}=g_{*}$. And in the case of the 2-torus, we also can show the reciprocal:

Proposition 2.9. Given $f, g \in \operatorname{Homeo}\left(\mathbb{T}^{2}\right)$, if $f$ and $g$ induce the same action in the closed curves of $\mathbb{T}^{2}$, that is $f_{*}=g_{*}$, then $f$ and $g$ are homotopic. 1

So, in the case of $S=\mathbb{T}^{2}$ that has genus $g=1$ we have that a map $f \in \operatorname{Homeo}\left(\mathbb{T}^{2}\right)$ induces an isomorphism $f_{*}$ that can be represented by an element of $\operatorname{GL}(2, \mathbb{Z})$.

And, conversely, a matrix $A \in \mathrm{GL}(2, \mathbb{Z})$ induces a linear automorphism of $\mathbb{R}^{2}$ such that $A \mathbb{Z}^{2}=\mathbb{Z}^{2}$. Thus, given $\check{z} \in \mathbb{R}^{2}$ and $\check{\pi}: \mathbb{R}^{2} \rightarrow \mathbb{T}^{2}$ the canonical universal covering projection, the map $\check{z} \mapsto A \check{z}$ projects, by $\check{\pi}$, onto a homeomorphism $f_{A}: \mathbb{T}^{2} \rightarrow \mathbb{T}^{2}$, that is said to be a linear automorphism of $\mathbb{T}^{2}$.

Now, if we consider the following equivalence class in Homeo( $\left.\mathbb{T}^{2}\right)$ :

$$
\text { given } f, g \in \operatorname{Homeo}\left(\mathbb{T}^{2}\right): f \sim g \Leftrightarrow f \text { is isotopic to } g
$$

and let Homeo $\left(\mathbb{T}^{2}\right) / \sim$ be the quotient space, the discussion above together with Theorem 2.8 imply that two elements $f, g \in \operatorname{Homeo}\left(\mathbb{T}^{2}\right)$ are isotopic if and only if $f_{*}=g_{*}$.

Therefore, the map $f \mapsto f_{*}$ induce a bijection

$$
\xi: \operatorname{Homeo}\left(\mathbb{T}^{2}\right) / \sim \rightarrow \operatorname{GL}(2, \mathbb{Z})
$$

and the inverse of this bijection is the map which associates an element $A$ of $\mathrm{GL}(2, \mathbb{Z})$ to the isotopy class of the homeomorphism $f_{A}$ (see Proposition 3.1.1 in [BLR20]).

From this, we have that any $f \in \operatorname{Homeo}\left(\mathbb{T}^{2}\right)$ is isotopic to a unique linear automorphism of $\mathbb{T}^{2}$ : the automorphism $f_{A}$ with $A=f_{*}$.

Given $A \in \mathrm{GL}(2, \mathbb{Z})$, there are essentially three kinds of different dynamical behaviors for the the linear automorphism $f_{A}$. We say that

\footnotetext{
${ }^{1}$ Is important to emphasize that this proposition is false for surfaces of larger genus. For more details see Chapter 2 of [BLR20].
} 
1. $f_{A}$ is a linear Anosov automorphism if the associated matrix $A$ has two real eigenvalues: the contracting eigenvalue $\left|\lambda_{-}\right|<1$ and the expanding eigenvalue $\left|\lambda_{+}\right|>1$;

2. $f_{A}$ is periodic if there is some integer $k \geq 1$ such that $f_{A}^{k}=\left.\operatorname{Id}\right|_{\mathbb{T}^{2}}$;

3. $f_{A}$ is reducible if $f_{A}$ preserves a simple closed curve on $\mathbb{T}^{2}$.

Indeed, to do this classification we can study the sign of the discriminant of the characteristic polynomial of $A \in \mathrm{GL}(2, \mathbb{Z})$, that is $\Delta=\operatorname{tr}(A)^{2}-4 \operatorname{det}(A)$. As $\operatorname{det}(A)= \pm 1$ we have the following cases:

a) If $\operatorname{det}(A)=1$ then $\Delta=\operatorname{tr}(A)^{2}-4$ and we have:

1. If $|\operatorname{tr}(A)|>2$ then $A$ has two distinct real eigenvalues: $\left|\lambda_{-}\right|<1$ and $\left|\lambda_{+}\right|>1$. Thus $f_{A}$ is a linear Anosov.

2. If $\operatorname{tr}(A) \in\{-1,0,1\}$ then $A$ has complexes eigenvalues $\lambda$ and $\bar{\lambda}$ such $\lambda^{k}=1$, for some integer $k \leq 6$. Therefore $f_{A}^{k}=\left.\operatorname{Id}\right|_{\mathbb{T}^{2}}$, so $f_{A}$ is periodic.

3. If $\operatorname{tr}(A)= \pm 2$ then $A$ has a double eigenvalue, that is either 1 or -1 . And in this case, we have that $f_{A}$ fix a simple closed curve of $\mathbb{T}^{2}$, so $f_{A}$ is reducible. Moreover, the matrix $A$ is conjugated to a matrix of type

$$
\left(\begin{array}{ll}
1 & k \\
0 & 1
\end{array}\right) \text { or }\left(\begin{array}{cc}
-1 & k \\
0 & -1
\end{array}\right) \text {. }
$$

For more details, see [BLR20].

Note that if $k=0$ then $f_{A}=\left.\operatorname{Id}\right|_{\mathbb{T}^{2}}$ or $f_{A}^{2}=\left.\operatorname{Id}\right|_{\mathbb{T}^{2}}$, respectively, and therefore $f_{A}$ is periodic. However, if $k \neq 0$ the automorphism $f_{A}$ for the first type is said to be a Dehn Twist, of order $k$. The second one is obtained by composing a Dehn twist with $-\left.\mathrm{Id}\right|_{\mathbb{T}^{2}}$.

b) If $\operatorname{det}(A)=-1$ then $\Delta=\operatorname{tr}(A)^{2}+4>0$ which means that $A$ has two real eigenvalues, and

1. If $\operatorname{tr}(A) \neq 0$ then their eigenvalues are such that $\left|\lambda_{-}\right|<1$ and $\left|\lambda_{+}\right|>1$, and thus $f_{A}$ is a linear Anosov.

2. If $\operatorname{tr}(A)=0$, the eigenvalues are 1 and -1 . Thus, is possible to show, the matrix $A$ is conjugated to a matrix of type

$$
\left(\begin{array}{cc}
1 & 0 \\
0 & -1
\end{array}\right)
$$

And, therefore $f_{A}^{2}=\left.\operatorname{Id}\right|_{\mathbb{T}^{2}}$, so $f_{A}$ is periodic. 
Therefore, given $f \in \operatorname{Homeo}\left(\mathbb{T}^{2}\right)$ we have that $f$ is isotopic to a linear automorphism $f_{A}$ that is one of the following types:

I. Linear Anosov;

II. Periodic and, in this case, there exist some integer $k \geq 1$ such that $f^{k}$ is isotopic to identity;

III. Reducible, but not periodic. So, in this case, there exist some integer $k \geq 1$ such that $f^{k}$ is isotopic to a Dehn Twist.

The next proposition provides a relation for homeomorphisms of $\mathbb{T}^{2}$ in the same isotopy class and their lifts to $\mathbb{R}^{2}$.

Proposition 2.10. Let $f \in \operatorname{Homeo}\left(\mathbb{T}^{2}\right), A \in \mathrm{GL}(2, \mathbb{Z})$ and $\check{f} \in \operatorname{Homeo}\left(\mathbb{R}^{2}\right)$ be a lift of $f$. The following statements are equivalent:

1. $f$ is isotopic to $f_{A}$;

2. there exists a continuous map $\varphi: \mathbb{T}^{2} \rightarrow \mathbb{R}^{2}$ such that

$$
\check{f}(\check{z})=A \check{z}+\varphi(\check{\pi}(\check{z})), \quad \forall \check{z} \in \mathbb{R}^{2}
$$

where $\check{\pi}: \mathbb{R}^{2} \rightarrow \mathbb{T}^{2}$ is the canonical universal covering projection of $T^{2}$.

3. $\check{f}(\check{z}+p)=\check{f}(\check{z})+A p$, where $p \in \mathbb{Z}^{2}$. 


\section{Chapter 3}

\section{Rotation Set Theory}

\subsection{2-Torus Homeomorphism}

Let $f \in \operatorname{Homeo}\left(\mathbb{T}^{2}\right)$ and $\check{\pi}: \mathbb{R}^{2} \rightarrow \mathbb{T}^{2}$ the canonical universal covering projection of $\mathbb{T}^{2}$. Take a lift $\check{f} \in \operatorname{Homeo}\left(\mathbb{R}^{2}\right)$ of $f$ to $\mathbb{R}^{2}$, that is $\check{f}$ satisfies

$$
\check{\pi} \circ \check{f}=f \circ \check{\pi} .
$$

It follows that, if $\check{f}, \check{f}^{\prime} \in \operatorname{Homeo}\left(\mathbb{R}^{2}\right)$ are two lifts of $f \in \operatorname{Homeo}\left(\mathbb{T}^{2}\right)$ then $\check{f}(\cdot)-\check{f}^{\prime}(\cdot)$ is a continuous map with values in $\mathbb{Z}^{2}$, thus constant.

Moreover, for the discussion of the previous chapter, we know that if $f \in \operatorname{Homeo}\left(\mathbb{T}^{2}\right)$ is isotopic to identity, then a lift $\check{f} \in \operatorname{Homeo}\left(\mathbb{R}^{2}\right)$ of $f$ commutes with the integer vector translations.

From this point on, and until the end of this section, we will assume that $f \in$ Homeo $\left(\mathbb{T}^{2}\right)$ is isotopic to identity. Remember that we denote as $\mathrm{Homeo}_{0}\left(\mathbb{T}^{2}\right)$ the subset of Homeo $\left(\mathbb{T}^{2}\right)$ of the homeomorphisms that are isotopic to identity.

The purpose of the rotation theory is to measure the average speed of any point in the lift. In this sense, given an $f \in \operatorname{Homeo}_{0}\left(\mathbb{T}^{2}\right)$, Misiurewicz and Ziemian in [MZ89] defined the rotation set of a lift $\check{f} \in \operatorname{Homeo}\left(\mathbb{R}^{2}\right)$ of $f$, denoted by $\rho(\check{f})$, as the set of all limit points of the following sequences

$$
\left(\frac{\check{f}^{n_{k}}\left(\check{z}_{k}\right)-\check{z}_{k}}{n_{k}}\right)_{k \geq 1}
$$

where $\left(n_{k}\right)_{k \geq 1}$ is an increasing sequence of integers and $\left(\check{z}_{k}\right)_{k \geq 1} \subset \mathbb{R}^{2}$.

If a point $z \in \mathbb{T}^{2}$ is such that the limit

$$
\lim _{n \rightarrow+\infty} \frac{\check{f}^{n}(\check{z})-\check{z}}{n} \text { exists, }
$$

where $\check{z} \in \check{\pi}^{-1}(z)$, then we define $\rho(z, \check{f})$ as the limit above and we call it the rotation 
vector of $z$. Since $f$ commutes with integer vector translations the above limit does not depend on the point $\check{z}$ chosen in $\check{\pi}^{-1}(z)$.

Note that the definition of rotation set is for some lift $\check{f} \in \operatorname{Homeo}\left(\mathbb{R}^{2}\right)$ of $f \in$ Homeo $_{0}\left(\mathbb{T}^{2}\right)$. However, we know that given another lift $\check{f}^{\prime} \in \operatorname{Homeo}\left(\mathbb{R}^{2}\right)$ of $f$, there is some $p^{\prime} \in \mathbb{Z}^{2}$ such that $\check{f}^{\prime}=\check{f}+p^{\prime}$. Moreover, it is not difficult to verify that the rotation set satisfies

$$
\rho\left(\check{f}^{q}+p\right)=q \rho(\check{f})+p,
$$

for any $p \in \mathbb{Z}^{2}$ and any $q \in \mathbb{N}$ (see [MZ89]). Therefore, $\rho\left(\check{f}^{\prime}\right)=\rho(\check{f})+p^{\prime}$. Meaning that given two lifts of $f$, the rotation set of one is an integer vector translation of the other.

Apart from Misiurewicz and Ziemian classical constructions, there are other ways to estimate rotation of points for homeomorphisms isotopic to identity of $\mathbb{T}^{2}$. For instance, instead of averaging the displacement for a single point, we can look at all points at once and average the spatial displacement. To do that, we use invariant measures.

More specifically, let $f \in \mathrm{Homeo}_{0}\left(\mathbb{T}^{2}\right)$. Denote by $\mathcal{M}(f)$ the set of all $f$-invariant Borel probability measures on $\mathbb{T}^{2}$ and $\mathcal{M}_{E}(f)$ its subset of ergodic measures. Let $\mu \in \mathcal{M}(f)$, the rotation number associated to $\mu$ is defined as

$$
\rho_{\mu}(\check{f})=\int_{\mathbb{T}^{2}} \varphi \mathrm{d} \mu
$$

where $\varphi: \mathbb{T}^{2} \rightarrow \mathbb{R}^{2}$ is the continuous map, known as the displacement function, given by Proposition 2.10, therefore $\varphi(z)=\check{f}(\check{z})-\check{z}$, for some $\check{z} \in \check{\pi}^{-1}(z)$ (which is independent of the choice). And we can define the set

$$
\rho_{m e s}(\check{f})=\left\{\int_{\mathbb{T}^{2}} \varphi \mathrm{d} \mu \mid \mu \in \mathcal{M}(f)\right\}
$$

The following proposition collects some results about the rotation set.

Proposition 3.1 (see [MZ89]). Let $f \in \operatorname{Homeo}_{0}\left(\mathbb{T}^{2}\right)$ and $\check{f} \in \operatorname{Homeo}\left(\mathbb{R}^{2}\right)$ be some lift of f. The following properties hold:

1. $\rho(\check{f})$ is a non-empty, closed, compact and convex set of $\mathbb{R}^{2}$;

2. If $\mu \in \mathcal{M}_{E}(f)$ is such that $\rho_{\mu}(\check{f})=a$ then for $\mu$-almost every point $z \in \mathbb{T}^{2}$ and any $\check{z} \in \check{\pi}^{-1}(z)$,

$$
\lim _{n \rightarrow \infty} \frac{\check{f}^{n}(\check{z})-\check{z}}{n}=a
$$

3. If $a \in \rho(\check{f})$ is an extremal point (in the sense of convex set) then there exists $\mu \in \mathcal{M}_{E}(f)$ such that $\rho_{\mu}(\check{f})=a$;

4. $\rho(\check{f})=\rho_{\text {mes }}(\check{f})$. 
We will say that a vector $v \in \mathbb{R}^{2}$ is rational, if both coordinates of $v$ are rationals numbers. Let $f \in \operatorname{Homeo}_{0}\left(\mathbb{T}^{2}\right)$ and $\check{f}$ a lift of $f$. We will say that a rational vector in the rotation set $v=\left(\frac{p_{1}}{q}, \frac{p_{2}}{q}\right) \in \rho(\check{f})$, such that $p_{1}, p_{2}$ and $q$ are integers mutually coprime (that means $\operatorname{gcd}\left(p_{1}, p_{2}, q\right)=1$ ), is realized by a periodic orbit if there exists $z \in \mathbb{T}^{2}$ such that

$$
\check{f}^{q}(\check{z})=\check{z}+\left(p_{1}, p_{2}\right)
$$

where $\check{z} \in \check{\pi}^{-1}(z)$ is independent of the choice. Note that this implies that $f^{q}(z)=z$ and

$$
\lim _{n \rightarrow+\infty} \frac{\check{f}^{n}(\check{z})-\check{z}}{n}=v
$$

Kocsard and Koropecki in [KK08] showed that under topological conjugacies, the rotation set has the following behavior:

Lemma 3.2 (Lemma 2.4 in $[\mathrm{KK} 08]$ ). Let $f, g \in \operatorname{Homeo}\left(\mathbb{T}^{2}\right)$ and $A \in \mathrm{GL}(2, \mathbb{Z})$. Suppose that $f \in \mathrm{Homeo}_{0}\left(\mathbb{T}^{2}\right)$ and $g$ is isotopic to $f_{A}$, the linear automorphism induced by $A$ on $\mathbb{T}^{2}$. Let $\check{f}$ and $\check{g}$ be respective lifts of $f$ and $g$ to $\mathbb{R}^{2}$, then

$$
\rho\left(\check{g} \circ \check{f} \circ \check{g}^{-1}\right)=A \rho(\check{f})
$$

In particular, $\rho\left(A \check{f} A^{-1}\right)=A \rho(\check{f})$.

Remark 3.3. The map $A \check{f} A^{-1}$ is a lift to $\mathbb{R}^{2}$ of a homeomorphism of $\mathbb{T}^{2}$ topologically conjugated to $f$, namely $f_{A} \circ f \circ f_{A}^{-1}$ where $f_{A}$ is the automorphism of $\mathbb{T}^{2}$ induced by $A$. Therefore, if we wish to prove the existence of a property for $f$ that is invariant by conjugation, Lemma 3.2 tell us that we can consider some simpler case: where the rotation set is the image of $\rho(\check{f})$ by some element of $\operatorname{GL}(2, \mathbb{Z})$.

The direction of a non-degenerate line segment $l$ is given by the diference between two distinct vectors $v_{1}, v_{2} \in l$ in the following way: if $v_{0}=v_{2}-v_{1}$ then, as $v_{0} \neq 0, v=\frac{v_{0}}{\left\|v_{0}\right\|}$ is the direction of $l$, and it is uniquely defined modulo a change of signal. We will say that $l$ is a non-degenerate line segment with rational slope if there is some $\lambda \in \mathbb{R} \backslash\{0\}$ such that the direction of $l$ is $v=\lambda\left(p_{1}, p_{2}\right)$ where $\left(p_{1}, p_{2}\right) \in \mathbb{Z} \backslash\{(0,0)\}$.

By a horizontal segment we mean a non-degenerate line segment whose its direction is given by $\pm(1,0)$. And, by a vertical segment we mean a non-degenerate line segment whose its direction is given by $\pm(0,1)$. Note that both horizontal and vertical segments are non-degenerate line segments with rational slope, by definition.

The next Corollary will be very useful in the sense of Remark 3.3.

Corollary 3.4. If $\rho(\check{f})$ is a non-degenerate line segment of rational slope then there is a matrix $A \in \mathrm{GL}(2, \mathbb{Z})$ such that $\rho\left(A \check{f} A^{-1}\right)$ is a horizontal segment.

Proof. Indeed, if $\rho(\check{f})$ has a rational slope then there are $p_{1}, p_{2} \in \mathbb{Z}$ coprimes and $\lambda \in$ $\mathbb{R} \backslash\{0\}$ such that the direction of $\rho(\check{f})$ is given by $v=\lambda\left(p_{1}, p_{2}\right)$. 
Then Bezout's Identity implies that there exist $x, y \in \mathbb{Z}$ such that $p_{1} x+p_{2} y=1$. Thus the matrix

$$
A=\left(\begin{array}{cc}
x & y \\
-p_{2} & p_{1}
\end{array}\right)
$$

is in $\operatorname{GL}(2, \mathbb{Z})$ (in fact, $A \in \operatorname{SL}(2, \mathbb{Z})$, because $\operatorname{det}(A)=1$ ), and $A v=\left(\begin{array}{l}\lambda \\ 0\end{array}\right)$. Therefore the direction of $A \rho(f)$ is given by $\frac{A v}{\|A v\|}= \pm(1,0)$, which is a horizontal segment, and by Lemma 3.2 , we have that $\rho\left(A \check{f} A^{-1}\right)=A \rho(f)$.

Let $\check{f} \in \operatorname{Homeo}\left(\mathbb{R}^{2}\right)$, we say that the deviations in the direction of some nonzero vector $v \in \mathbb{R}^{2}$ are uniformly bounded, if there exists a real number $M>0$ such that

$$
\left|\mathrm{p}_{v}\left(\check{f}^{n}(\check{w})-\check{w}\right)\right| \leq M, \quad \forall n \in \mathbb{Z} \text { and } \forall \check{w} \in \mathbb{R}^{2}
$$

where $\mathrm{p}_{v}(\cdot)=\left\langle\cdot, \frac{v}{\|v\|}\right\rangle$.

Definition 3.5. Let $f \in \mathrm{Homeo}_{0}\left(\mathbb{T}^{2}\right)$. We say that $f$ is annular if there is an integer $k \geq 1$ and a lift $\check{g} \in \operatorname{Homeo}\left(\mathbb{R}^{2}\right)$ of $g=f^{k}$ such that the deviations in the direction of some nonzero integer vector $v \in \mathbb{Z}^{2}$ are uniformly bounded.

The next theorem is an important result due to Dávalos, [Dáv18, and it will be very useful in the proof of Theorem A and Proposition C.

Theorem 3.6 (Theorem A in [Dáv18]). If $\rho(\check{f})$ is a non-degenerate line segment with rational slope containing rational points, then $f$ is annular in the direction perpendicular to $\rho(\check{f})$.

As a consequence of this theorem we have the following:

Corollary 3.7. If $\rho(\check{f})$ is a non-degenerate line segment with rational slope containing the origin and such that $(0,0) \in \rho(\check{f})$ is realized then there is a lift $\check{f}$ of $f$ which has uniformly bounded deviations in the direction perpendicular to $\rho(\check{f})$.

Proof. Let $v=\left(v_{1}, v_{2}\right) \in \mathbb{Z}^{2} \backslash\{(0,0)\}$ be a multiple of the direction of $\rho(\check{f})$ and let $v^{\perp}=\left(-v_{2}, v_{1}\right)$. By Theorem 3.6 as $\rho(\check{f})$ is a non-degenerate line segment with rational slope containing the origin then there is an integer $k \geq 1$ and a lift $\check{g}: \mathbb{R}^{2} \rightarrow \mathbb{R}^{2}$ of $g=f^{k}$ such that the deviations in the direction perpendicular to $\rho(\check{f})$ are uniformly bounded, that is

$$
\left|\mathrm{p}_{v^{\perp}}\left(\check{g}^{n}(\check{w})-\check{w}\right)\right| \leq M, \quad \forall n \in \mathbb{Z} \text { and } \forall \check{w} \in \mathbb{R}^{2} .
$$

Claim. The lift $\check{g} \in \operatorname{Homeo}\left(\mathbb{R}^{2}\right)$ of $g=f^{k}$ can be considered as $\check{g}=\check{f}^{k}$.

Proof of Claim. Otherwise, then there is $p \in \mathbb{Z}^{2} \backslash\{(0,0)\}$ such that $\check{g}=\check{f}^{k}+p$ has uniformly bounded deviations in the direction perpendicular to the rotation set. And then, we have two possibilities: 
a) If $p=\lambda v$, for some $\lambda \in \mathbb{R}$.

As $\mathrm{p}_{v^{\perp}}(\lambda v)=0$ we have that

$$
\left|\mathrm{p}_{v^{\perp}}\left(\check{g}^{n}(\check{w})-\check{w}\right)\right|=\left|\mathrm{p}_{v^{\perp}}\left(\left(\check{f}^{k}\right)^{n}(\check{w})-\check{w}\right)\right|
$$

which implies that $\check{f}^{k}$ has uniformly bounded deviations in the direction perpendicular to $\rho(\check{f})$. And therefore, there is no loss of generality suppose that $\check{g}=\check{f}^{k}$.

b) If $p \in \mathbb{Z}^{2} \backslash\{\lambda v \mid \lambda \in \mathbb{R}\}$.

By assumption we have that $(0,0) \in \rho(\check{f})$ is realized. So, there is a point $z_{0} \in \operatorname{fix}(f)$ such that $\check{f}\left(\check{z}_{0}\right)=\check{z}_{0}$, where $\check{z}_{0} \in \check{\pi}^{-1}\left(z_{0}\right)$ is independent of the choice. This implies that $\check{g}\left(\check{z}_{0}\right)=\check{z}_{0}+p$ and $\left|\mathrm{p}_{v^{\perp}}\left(\check{g}^{n}\left(\check{z}_{0}\right)-\check{z}_{0}\right)\right|=n\left|\mathrm{p}_{v^{\perp}}(p)\right| \neq 0$. This means that there is no uniformly bounded deviations in the direction perpendicular to the rotation set. Therefore, this case does not occurs.

End of the proof of Corollary 3.7. So, let $\check{g}=\check{f}^{k}$ such that there is a real number $M>0$ such that

$$
\left|\mathrm{p}_{v^{\perp}}\left(\check{g}^{n}(\check{w})-\check{w}\right)\right| \leq M, \quad \forall n \in \mathbb{Z} \text { and } \forall \check{w} \in \mathbb{R}^{2}
$$

For all $n \in \mathbb{N}$ there is $m \in \mathbb{N}$ and $r \in\{0,1, \cdots, k-1\}$ such that $n=m k+r$. As $r \in\{0,1, \cdots, k-1\}$ is finite we have that $\left|\mathrm{p}_{v^{\perp}}\left(\check{f}^{r}(\check{w})-\check{w}\right)\right| \leq M_{0}$ for some constant $M_{0}>0$ and for all $r \in\{0,1 \cdots, k-1\}$. Therefore,

$$
\begin{aligned}
\left|\mathrm{p}_{v^{\perp}}\left(\check{f}^{n}(\check{w})-\check{w}\right)\right| & =\left|\mathrm{p}_{v^{\perp}}\left(\check{f}^{m k}\left(\check{f}^{r}(\check{w})\right)-\check{w}\right)\right| \\
& =\left|\mathrm{p}_{v^{\perp}}\left(\check{g}^{m}\left(\check{f}^{r}(\check{w})\right)-\check{f}^{r}(\check{w})\right)+\mathrm{p}_{v^{\perp}}\left(\check{f}^{r}(\check{w})-\check{w}\right)\right| \\
& \leq\left|\mathrm{p}_{v^{\perp}}\left(\check{g}^{m}\left(\check{f}^{r}(\check{w})\right)-\check{f}^{r}(\check{w})\right)\right|+\left|\mathrm{p}_{v^{\perp}}\left(\check{f}^{r}(\check{w})-\check{w}\right)\right| \\
& \leq M+M_{0}, \quad \forall n \in \mathbb{Z} \text { and } \forall \check{w} \in \mathbb{R}^{2} .
\end{aligned}
$$

Which means that $\check{f}$ has uniformly bounded deviations in the direction perpendicular to $\rho(\check{f})$.

\subsection{Annulus Homeomorphism}

Let us now consider the annulus $\mathbb{A}=\mathbb{T}^{1} \times \mathbb{R}, \hat{f} \in \operatorname{Homeo}(\mathbb{A})$ and $\check{\tau}: \mathbb{R}^{2} \rightarrow \mathbb{A}$ its canonical universal covering projection. Take a lift $\check{f} \in \operatorname{Homeo}\left(\mathbb{R}^{2}\right)$ of $\hat{f}$ to $\mathbb{R}^{2}$, that is $\check{f}$ satisfies

$$
\hat{f} \circ \check{\tau}=\check{\tau} \circ \check{f} .
$$

We have that given two lifts $\check{f}, \check{f}^{\prime} \in \operatorname{Homeo}\left(\mathbb{R}^{2}\right)$ of $\hat{f} \in \operatorname{Homeo}(\mathbb{A})$, there is some $p_{1} \in \mathbb{Z}$ such that

$$
\check{f}^{\prime}(\check{z})-\check{f}(\check{z})=\left(p_{1}, 0\right), \forall \check{z} \in \mathbb{R}^{2} .
$$


Moreover, there is a unique $\bar{p}_{1} \in\{-1,1\}$ such that

$$
\check{f}(\cdot+(1,0))=\check{f}(\cdot)+\left(\bar{p}_{1}, 0\right) .
$$

In particular, if $\hat{f} \in \operatorname{Homeo}(\mathbb{A})$ is isotopic to identity and if $\check{f} \in \operatorname{Homeo}\left(\mathbb{R}^{2}\right)$ is a lift of $\hat{f}$ then $\check{f}(\check{z}+(1,0))=\check{f}(\check{z})+(1,0)$ for any $\check{z} \in \mathbb{R}^{2}$, which implies that

$$
\check{f}\left(\check{z}+\left(p_{1}, 0\right)\right)=\check{f}(\check{z})+\left(p_{1}, 0\right), \forall \check{z} \in \mathbb{R}^{2}, \text { and } \forall p_{1} \in \mathbb{Z}
$$

that is, $\check{f}$ commutes with the integer horizontal translations.

From this point on, and until the end of this section, we will assume that $\hat{f} \in$ $\operatorname{Homeo}(\mathbb{A})$ is isotopic to identity.

Let $\hat{f} \in \operatorname{Homeo}_{0}(\mathbb{A})$. We define the set $\mathrm{ne}^{+}(\hat{f})$ as the set of all points $\hat{z} \in \mathbb{A}$ such its $\omega$-limit set is non-empty, ne ${ }^{-}(\hat{f})=\operatorname{ne}^{+}\left(\hat{f}^{-1}\right)$ as the set of all points $\hat{z} \in \mathbb{A}$ such its $\alpha$-limit set is non-empty and, finally $\operatorname{ne}(\hat{f})=\mathrm{ne}^{+}(\hat{f}) \cup \mathrm{ne}^{-}(\hat{f})$.

Let $\hat{f} \in \operatorname{Homeo}_{0}(\mathbb{A})$ and take a lift $\check{f} \in \operatorname{Homeo}\left(\mathbb{R}^{2}\right)$ of $\hat{f}$ to $\mathbb{R}^{2}$. As defined by Le Roux [LR13] and Conejeros [Con18] we will say that a point $\hat{z} \in \mathrm{ne}^{+}(\hat{f})$ has a rotation number $\operatorname{rot}(\hat{z}, \check{f})$ if for every compact set $\hat{K} \subset \mathbb{A}$ and every increasing sequence of integers $\left(n_{k}\right)_{k \geq 1}$ such that $\hat{f}^{n_{k}}(\hat{z}) \in \hat{K}$, we have

$$
\lim _{k \rightarrow \infty} \frac{\mathrm{p}_{1}\left(\check{f}^{n_{k}}(\check{z})-\check{z}\right)}{n_{k}}=\operatorname{rot}(\hat{z}, \check{f})
$$

where $\check{z} \in \check{\tau}^{-1}(\hat{z})$ is independent of the choice.

A loop in $\mathbb{A}$ will be called essential if it is not homotopic to zero.

Lemma 3.8. Let $\hat{I}=\left(\hat{f}_{t}\right)_{t \in \mathbb{R}}$ be an identity isotopy of $\hat{f}$. Let $\check{I}=\left(\check{f}_{t}\right)_{t \in \mathbb{R}}$ be the lift of $\hat{I}$ to $\mathbb{R}^{2}$ such that $\check{f}_{0}=\operatorname{Id}$ and $\check{f}:=\check{f}_{1}$ is the lift of $\hat{f}$ to $\mathbb{R}^{2}$ given by $\hat{I}$. Let $\hat{z} \in \mathbb{A}$ be a periodic point for $\hat{f}$ with period $q \geq 1$. Then

1. If its trajectory by the isotopy $\hat{I}^{q}(\hat{z})$ is homotopic to zero in $\mathbb{A}$ then its rotation number $\operatorname{rot}(\hat{z}, \check{f})$ is zero;

2. In the other hand, if $\hat{I}^{q}(\hat{z})$ is an essential loop in $\mathbb{A}$ then its rotation number $\operatorname{rot}(\hat{z}, \hat{f})$ is a non-zero rational number.

Proof. Let $\hat{z} \in \mathbb{A}$ such that $\hat{f}^{q}(\hat{z})=\hat{z}$, thus $\omega_{\hat{f}}(\hat{z}) \neq \emptyset$. Let $\check{f} \in \operatorname{Homeo}\left(\mathbb{R}^{2}\right)$ be the lift of $\hat{f}$ to $\mathbb{R}^{2}$ given by the isotopy $\hat{I}$, then there is some $p \in \mathbb{Z}$ such that

$$
\check{f}^{q}(\check{z})=\check{z}+(p, 0)
$$

where $\check{z} \in \check{\tau}^{-1}(\hat{z})$ is independent of the choice.

For each $n \in \mathbb{N}$ there exists $m \in \mathbb{N}$ and $r \in\{0, \cdots, q-1\}$ such that $n=m q+r$. Thus

$$
\left.\check{f}^{n}(\check{z})=\check{f}^{r+m q}(\check{z})\right)=\check{f}^{r}(\check{z})+m(p, 0) .
$$


Now, let $\hat{K} \subset \mathbb{A}$ be a compact set and $\left(n_{k}\right)_{k \geq 1}$ be an increasing sequence such that $\hat{f}^{n_{k}}(\hat{z}) \in \hat{K}$. Thus, if $n_{k}=m_{k} q+r_{k}$, where $m_{k} \in \mathbb{N}$ and $r_{k} \in\{0, \cdots, q-1\}$, we have by equation 3.3 that

$$
\frac{\mathrm{p}_{1}\left(\check{f}^{n_{k}}(\check{z})-\check{z}\right)}{n_{k}}=\frac{m_{k} p+\mathrm{p}_{1}\left(\check{f}^{r_{k}}(\check{z})-\check{z}\right)}{m_{k} q+r_{k}}
$$

As the sequence $\left(r_{k}\right)_{k \geq 1}$ is bounded and $\left(m_{k}\right)_{k \geq 1}$ is an increasing sequence, when $k$ goes to infinity we have

$$
\begin{aligned}
\lim _{k \rightarrow \infty} \frac{1}{n_{k}}\left(\mathrm{p}_{1}\left(\check{f}^{n_{k}}(\check{z})-\check{z}\right)\right) & =\lim _{k \rightarrow \infty} \frac{m_{k} p+\mathrm{p}_{1}\left(\check{f}^{r_{k}}(\check{z})-\check{z}\right)}{m_{k} q+r_{k}} \\
& =\lim _{k \rightarrow \infty}\left(\frac{m_{k} p}{m_{k} q}+\frac{\mathrm{p}_{1}\left(\check{f}^{r_{k}}(\check{z})-\check{z}\right)}{m_{k} q}\right) \cdot \frac{1}{1+\frac{r_{k}}{m_{k} q}} \\
& =\frac{p}{q}=\operatorname{rot}(\hat{z}, \check{f}) .
\end{aligned}
$$

1. If the loop $\hat{I}^{q}(\hat{z})$ is homotopic to zero in $\mathbb{A}$ then in 3.2 we have that $p=0$ which implies $\operatorname{rot}(\hat{z}, \check{f})=0$.

2. In the other hand, if $\hat{I}^{q}(\hat{z})$ is essential, then in 3.2 we have that $p \in \mathbb{Z} \backslash\{0\}$, and so $\operatorname{rot}(\hat{z}, \check{f}) \in \mathbb{Q} \backslash\{0\}$.

The last two results of this chapter, due to Le Calvez and Tal see [LCT18b], are related with topological horseshoe for annulus homeomorphism isotopic to identity and rotation set theory.

The first one ensure that if $\hat{f}$ is a homeomorphism isotopic to identity of $\mathbb{A}$ without a topological horseshoe, then every point $\hat{z} \in \mathrm{ne}^{+}(\hat{f})$ has a well-defined rotation number.

Theorem 3.9 (Theorem A in [LCT18b]). Let $\hat{f}$ be a homeomorphism isotopic to identity on $\mathbb{A}$ and $\check{f}$ a lift of $\hat{f}$ for $\mathbb{R}^{2}$. Suppose that $\hat{f}$ has no topological horseshoe, then

1. each point $\hat{z} \in \operatorname{ne}^{+}(\hat{f})$ has a well-defined rotation number $\operatorname{rot}(\hat{z}, \check{f})$;

2. for all points $\hat{z}, \hat{z}^{\prime}$ such that $\omega_{\hat{f}}(\hat{z})$ and $\omega_{\hat{f}}\left(\hat{z}^{\prime}\right)$ are non-empty, and such that $\hat{z}^{\prime} \in$ $\omega_{\hat{f}}(\hat{z})$, we have $\operatorname{rot}\left(\hat{z}^{\prime}, \check{f}\right)=\operatorname{rot}(\hat{z}, \check{f})$.

3. if $z \in \mathrm{ne}^{+}(\hat{f}) \cap \mathrm{ne}^{+}\left(\hat{f}^{-1}\right)$ is non-wandering, then $\operatorname{rot}\left(\hat{z}, \check{f}^{-1}\right)=-\operatorname{rot}(\hat{z}, \check{f})$.

4. the map $\operatorname{rot}_{\breve{f} \pm}: \Omega(\hat{f}) \cap \operatorname{ne}(\hat{f}) \rightarrow \mathbb{R}$ is continuous, where

$$
\operatorname{rot}_{\check{f}^{ \pm}}(\hat{z})= \begin{cases}\operatorname{rot}(\hat{z}, \check{f}) & \text { if } \hat{z} \in \Omega(\hat{f}) \cap \mathrm{ne}^{+}(\hat{f}) \\ -\operatorname{rot}\left(\hat{z}, \check{f}^{-1}\right) & \text { if } \hat{z} \in \Omega(\hat{f}) \cap \mathrm{ne}^{-}(\hat{f}) .\end{cases}
$$

The second result is expressed in terms of Birkhoff recurrence classes, remember Definition 2.3 , 
Let $\hat{f} \in \operatorname{Homeo}(\mathbb{A})$ be isotopic to the identity, we will denote $\hat{f}_{\text {sphere }}$ as the continuous extension of $\hat{f}$ to the sphere obtained by adding the two ends $N$ (superior end) and $S$ (inferior end) of $\mathbb{A}$.

Proposition 3.10 (Proposition D in [LCT18b]). Let $\hat{f}$ be a homeomorphism of $\mathbb{A}$ isotopic to the identity, $\check{f}$ a lift of $\hat{f}$ to $\mathbb{R}^{2}$. We suppose that:

1. $\check{f}$ has at least two rotation numbers well defined and distinct;

2. $N$ and $S$ belong to the same Birkhoff recurrence class of $\hat{f}_{\text {sphere }}$.

Then $\hat{f}$ has a topological horseshoe. 


\section{Chapter 4}

\section{Forcing Theory}

Let $S$ be an oriented surface. In Chapter 2 we defined a path on $S$ as a continuous map $\gamma: J \rightarrow S$ defined on an interval $J \subset \mathbb{R}$. From now on, in absence of ambiguity, its image will also be called a path and denoted by $\gamma$. We will denote $\gamma^{-1}:-J \rightarrow S$ the path defined by $\gamma^{-1}(t)=\gamma(-t)$. If $X$ and $Y$ are two disjoint subset of $S$ we will say that a path $\gamma:[a, b] \rightarrow S$ joins $X$ to $Y$ if $\gamma(a) \in X$ and $\gamma(b) \in Y$. Moreover, if $\gamma:[a, b] \rightarrow S$ and $\gamma^{\prime}:\left[a^{\prime}, b^{\prime}\right] \rightarrow S$ are two paths such that $\gamma(b)=\gamma^{\prime}\left(a^{\prime}\right)$ then we can concatenate the two paths and define the path $\gamma \gamma^{\prime}:\left[a, b+b^{\prime}-a^{\prime}\right] \rightarrow S$

$$
\gamma \gamma^{\prime}(t)= \begin{cases}\gamma(t) & \text { if } t \in[a, b] \\ \gamma^{\prime}\left(t-b+a^{\prime}\right) & \text { if } t \in\left[b, b+b^{\prime}-a^{\prime}\right]\end{cases}
$$

A path $\gamma: J \rightarrow S$ is proper if $J$ is open and the preimage of every compact subset of $S$ is compact. If $S=\mathbb{R}^{2}$, a line is an injective and proper path $\phi: J \rightarrow \mathbb{R}^{2}$, it inherits a natural orientation induced by the usual orientation on $\mathbb{R}$. Moreover, for the Jordan-Schoenflies Theorem (see [Hat02]) the complement of a line $\phi$ has two connected components, we will denote $R(\phi)$ for the one that is on the right of $\phi$ and $L(\phi)$ for the other one that is on the left of it.

A path $\gamma: \mathbb{R} \rightarrow S$ such that $\gamma(t+1)=\gamma(t)$ for every $t \in \mathbb{R}$ lifts a continuous map $\Gamma: \mathbb{T}^{1} \rightarrow S$. We will say that $\Gamma$ is a loop and $\gamma$ is its natural lift. When the map $\Gamma$ is also injective then $\Gamma$ will be called simple loop. If $n \geq 1$, we denote $\Gamma^{n}$ the loop lifted by the path $t \mapsto \gamma(n t)$, and we will say that $\Gamma^{n}$ is a multiple of the loop $\Gamma$.

\subsection{Singular Oriented Foliations}

By a singular oriented foliation on an oriented surface $S$ we mean a closed set $\operatorname{sing}(\mathcal{F})$, called the set of singularities, together with a topological oriented foliation $\mathcal{F}^{\prime}$ on the complement of $\operatorname{sing}(\mathcal{F})$, that we will denote by $\operatorname{dom}(\mathcal{F}):=S \backslash \operatorname{sing}(\mathcal{F})$ and call it domain of $\mathcal{F}$. 
Namely, $\mathcal{F}^{\prime}$ is a partition of $\operatorname{dom}(\mathcal{F})$ into connected oriented 1-manifolds (circles or lines) called (regular) leaves of $\mathcal{F}$, such that for every $z \in \operatorname{dom}(\mathcal{F})$ there exists a neighborhood $W$ of $z$ and an oriented preserving homeomorphism $h$ mapping $W$ to $(0,1)^{2} \subset \mathbb{R}^{2}$. Moreover, $h$ maps the restricted foliation $\left.\mathcal{F}\right|_{W}$ onto the oriented foliation by vertical lines oriented downwards. We say that $W$ and $h$ are a trivialization neighborhood and a trivialization chart at the point $z$, respectively. For every $z \in \operatorname{dom}(\mathcal{F})$ we will write $\phi_{z}$ for the leaf that contains $z$.

If $\operatorname{sing}(\mathcal{F})$ is an empty set we will say that $\mathcal{F}$ is a non-singular oriented foliation.

By Whitney's Theorem, see [Whi33] and [Whi41, any singular oriented foliation $\mathcal{F}$ can be embedded in a flow, which means that $\mathcal{F}$ is the set of oriented orbits of some topological flow $\Phi: S \times \mathbb{R} \rightarrow S$, where the singularities of $\mathcal{F}$ coincides with the set of the fixed points of $\Phi$. Thus we can define the $\omega$-limit and $\alpha$-limit sets of a leaf $\phi$ as follows: if $\phi$ is a leaf of $\mathcal{F}$ and $z \in \phi$ then

$$
\alpha(\phi)=\bigcap_{n \geq 0} \overline{\{\Phi(z, t) ; t \leq n\}} \text { and } \omega(\phi)=\bigcap_{n \geq 0} \overline{\{\Phi(z, t) ; t \geq n\}}
$$

We will denote by $\phi_{z}^{-}$the negative half-leaf and $\phi_{z}^{+}$the positive half-leaf from the point $z$, which means that if $\phi_{z}=\Phi(z, 0)$ then

$$
\phi_{z}^{-}=\{\Phi(z, t) ; t<0\} \text {, and } \phi_{z}^{+}=\{\Phi(z, t) ; t>0\}
$$

If $\breve{S}$ is the universal covering space of $S$ and $\breve{\pi}: \breve{S} \rightarrow S$ a universal covering projection then $\mathcal{F}$ can be naturally lifted to a singular foliation $\breve{\mathcal{F}}$ of $\breve{S}$ such that

$$
\operatorname{dom}(\breve{\mathcal{F}})=\breve{\pi}^{-1}(\operatorname{dom}(\mathcal{F}))
$$

Moreover, let $\operatorname{Deck}(S)$ be the set of the covering automorphism of $S$. If $\breve{\phi} \in \operatorname{dom}(\breve{\mathcal{F}})$ then $T \breve{\phi} \in \operatorname{dom}(\breve{\mathcal{F}})$ and, equivalently, if $\breve{z} \in \operatorname{sing}(\breve{\mathcal{F}})$ then $T \breve{z} \in \operatorname{sing}(\breve{\mathcal{F}})$, for every $T \in \operatorname{Deck}(S)$. In particular, we have for the $\omega$-limit and $\alpha$-limit sets of a leaf $\breve{\phi} \in \breve{\pi}^{-1}(\phi)$ that

$$
\omega(T \breve{\phi})=T \omega(\breve{\phi}) \text { and } \alpha(T \breve{\phi})=T \alpha(\breve{\phi}) \text {, for every } T \in \operatorname{Deck}(S)
$$

As $\operatorname{dom}(\mathcal{F})$ is an open set on $S$, it is itself an oriented surface (not necessarily connected). So, in the whole text we will denote $\widetilde{\operatorname{dom}}(\mathcal{F})$ as the universal covering space of $\operatorname{dom}(\mathcal{F}), \tilde{\pi}: \widetilde{\operatorname{dom}}(\mathcal{F}) \rightarrow \operatorname{dom}(\mathcal{F})$ the universal covering projection and $\tilde{\mathcal{F}}$ the non-singular oriented foliation of $\widetilde{\operatorname{dom}}(\mathcal{F})$ lifted from $\left.\mathcal{F}\right|_{\operatorname{dom}(\mathcal{F})}$.

\section{$4.2 \mathcal{F}$-Transverse paths}

Let $\mathcal{F}$ be a singular oriented foliation on an oriented surface $S$. A path $\gamma: J \rightarrow S$ is positively transverse to $\mathcal{F}$ (which we will say just $\mathcal{F}$-transverse) if the image of $\gamma$ does not 
contain any singularity of $\mathcal{F}$, and each intersection of $\gamma$ with a leaf of $\mathcal{F}$ is topologically transverse and cross the leaf locally "from right to left". More precisely,

Definition 4.1. We will say that a path $\gamma: J \rightarrow \operatorname{dom}(\mathcal{F})$ is positively transverse to $\mathcal{F}$ $(\mathcal{F}$-transverse $)$ if for every $t_{0} \in J$ and a trivialization chart at $\gamma\left(t_{0}\right)$ the first coordinate of the map $t \mapsto h(\gamma(t))$ is strictly increasing in a neighborhood of $t_{0}$.

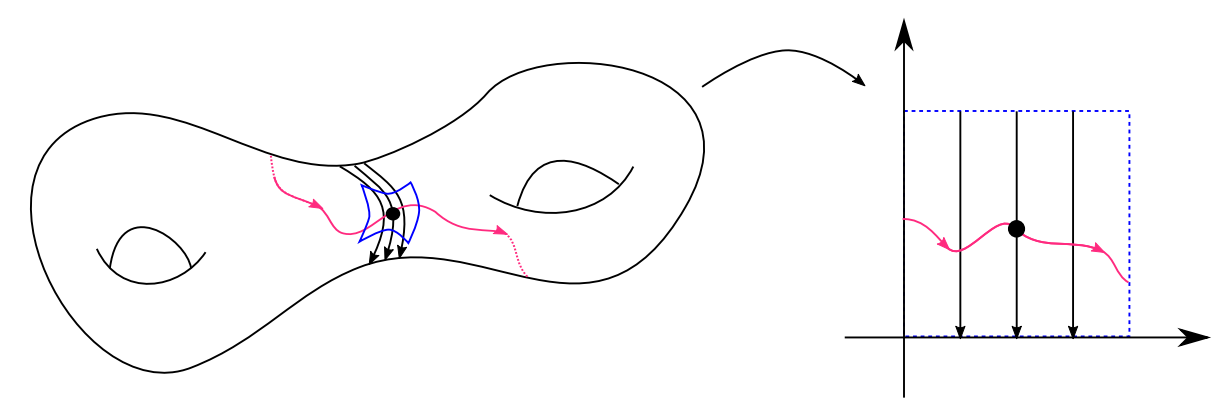

Figure 4.1: $\gamma$ is positively transverse to $\mathcal{F}$.

When $\mathcal{F}$ is a non-singular oriented foliation on $\mathbb{R}^{2}$ then Definition 4.1 is equivalent to saying that for all $t_{0} \in J$

$$
\left\{\gamma(t) \mid t \in J \text { and } t<t_{0}\right\} \subset R\left(\phi_{\gamma\left(t_{0}\right)}\right) \quad \text { and } \quad\left\{\gamma(t) \mid t \in J \text { and } t_{0}<t\right\} \subset L\left(\phi_{\gamma\left(t_{0}\right)}\right) \text {. }
$$

An important definition is about an equivalence relation between $\mathcal{F}$-transverse paths. For this definition, suppose first that $\mathcal{F}$ is a non-singular oriented foliation of $\mathbb{R}^{2}$. We say that two $\mathcal{F}$-transvese paths $\gamma$ and $\gamma^{\prime}$ are $\mathcal{F}$-equivalent if there exists an increasing homeomorphism $h: J \rightarrow J^{\prime}$ such that $\phi_{\gamma^{\prime}(h(t))}=\phi_{\gamma(t)}$, for every $t \in J$. Observe that this definition is equivalent to say that $\gamma$ and $\gamma^{\prime}$, meet exactly the same leaves of $\mathcal{F}$.

For the general case, we have the following:

Definition 4.2. Let $\gamma: J \rightarrow \operatorname{dom}(\mathcal{F})$ and $\gamma^{\prime}: J^{\prime} \rightarrow \operatorname{dom}(\mathcal{F})$ be two $\mathcal{F}$-transverse paths. We will say that $\gamma$ and $\gamma^{\prime}$ are $\mathcal{F}$-equivalent if they can be lifted to $\widetilde{\operatorname{dom}}(\mathcal{F})$ into paths $\tilde{\gamma}: J \rightarrow \widetilde{\operatorname{dom}}(\mathcal{F})$ and $\tilde{\gamma}^{\prime}: J^{\prime} \rightarrow \widetilde{\operatorname{dom}}(\mathcal{F})$, respectively, that are $\tilde{\mathcal{F}}$-equivalents.

A loop $\Gamma: \mathbb{T}^{1} \rightarrow \operatorname{dom}(\mathcal{F})$ is $\mathcal{F}$-transverse if it is the case for its natural lift $\gamma:$ $\mathbb{R} \rightarrow \operatorname{dom}(\mathcal{F})$. And, two $\mathcal{F}$-transverse loops $\Gamma$ and $\Gamma^{\prime}$ are $\mathcal{F}$-equivalent if there exists two lifts $\tilde{\gamma}: \mathbb{R} \rightarrow \widetilde{\operatorname{dom}}(\mathcal{F})$ and $\tilde{\gamma}^{\prime}: \mathbb{R} \rightarrow \widetilde{\operatorname{dom}}(\mathcal{F})$ of $\gamma$ and $\gamma^{\prime}$ the natural lifts of $\Gamma$ and $\Gamma^{\prime}$ respectively, such that for every $[a, b] \subset \mathbb{R}$ there exists $\left[a^{\prime}, b^{\prime}\right] \subset \mathbb{R}$ we have that $\left.\tilde{\gamma}\right|_{[a, b]}$ and $\left.\tilde{\gamma}^{\prime}\right|_{\left[a^{\prime}, b^{\prime}\right]}$ are $\tilde{\mathcal{F}}$-equivalents.

Moreover, we have that $\Gamma^{n}$ and $\Gamma^{\prime n}$ are $\mathcal{F}$-equivalent loops, for every $n \geq 1$, if it is the case for $\Gamma$ and $\Gamma^{\prime}$. An $\mathcal{F}$-transverse loop $\Gamma$ is called prime if there is no $\mathcal{F}$-transverse loop $\Gamma^{\prime}$ and an integer $n \geq 2$ such that $\Gamma$ is $\mathcal{F}$-equivalent to $\Gamma^{\prime} n$. Note that if $\Gamma$ is a simple loop then it is prime. 
Definition 4.3. An $\mathcal{F}$-transverse path $\gamma: \mathbb{R} \rightarrow S$ will be called $\mathcal{F}$-positively recurrent if for every interval $J \subset \mathbb{R}$ and every $t \in \mathbb{R}$ there exists an interval $J_{0} \subset[t,+\infty)$ such that $\left.\gamma\right|_{J_{0}}$ is equivalent to $\left.\gamma\right|_{J}$. It will be called $\mathcal{F}$-negatively recurrent if for every interval $J \subset \mathbb{R}$ and every $t \in \mathbb{R}$ there exists an interval $J_{0} \subset(-\infty, t]$ such that $\left.\gamma\right|_{J_{0}}$ is equivalent to $\left.\gamma\right|_{J}$. It is $\mathcal{F}$-bi-recurrent if it is both $\mathcal{F}$-positively and $\mathcal{F}$-negatively recurrent.

Moreover, this definition is preserved in the equivalence class of a path, that is, if $\gamma: \mathbb{R} \rightarrow S$ and $\gamma_{0}: \mathbb{R} \rightarrow S$ are $\mathcal{F}$-equivalent and if $\gamma$ is $\mathcal{F}$-positively recurrent (or $\mathcal{F}$-negatively recurrent), so is $\gamma_{0}$.

As an example of an $\mathcal{F}$-bi-recurrent path is the natural lift of an $\mathcal{F}$-transverse loop.

\subsection{1 $\mathcal{F}$-transverse intersection}

Until the end of this subsection we will give a precise definition of a central tool on forcing theory for transverse trajectories, which is the $\mathcal{F}$-transverse intersection of two $\mathcal{F}$-transverse paths.

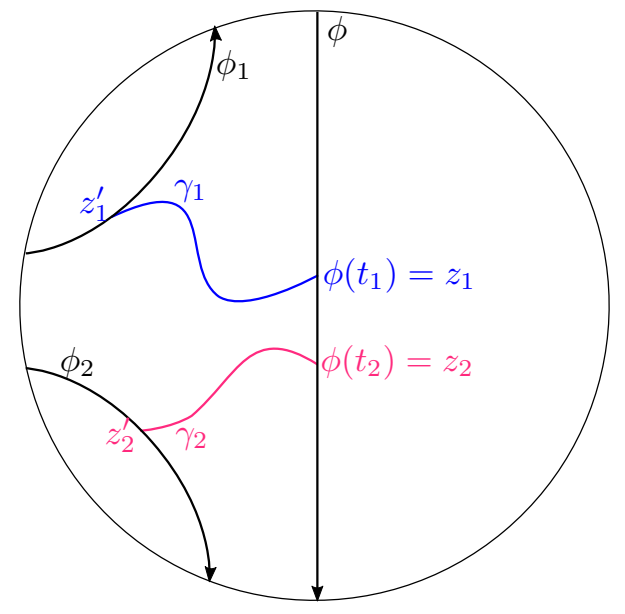

Figure 4.2: $\phi_{2}$ is above $\phi_{1}$ relative to $\phi$.

Definition 4.4. Given three disjoint lines $\phi, \phi_{1}, \phi_{2}: \mathbb{R} \rightarrow \mathbb{R}^{2}$, we will say that $\phi_{2}$ is above $\phi_{1}$ relative to $\phi$ (and that $\phi_{1}$ is below $\phi_{2}$ relative to $\phi$ ) if none of the lines separates the two others and if, for every pair of disjoint paths $\gamma_{1}$ and $\gamma_{2}$ joining $z_{1}=\phi\left(t_{1}\right)$ to $z_{1}^{\prime} \in \phi_{1}$ and $z_{2}=\phi\left(t_{2}\right)$ to $z_{2}^{\prime} \in \phi_{2}$, respectively, such that the paths do not meet the lines but at their endpoints, we have that $t_{1}<t_{2}$. See the Figure 4.2 .

Note that this definition does not depend on the orientation of the lines $\phi_{1}$ and $\phi_{2}$ neither of the orientation of the paths $\gamma_{1}$ or $\gamma_{2}$. It only depends of the orientation of $\phi$.

Before we give the definition of $\mathcal{F}$-transverse intersection in the general case, let us define it for the case when $\mathcal{F}$ is a non-singular oriented foliation on $S=\mathbb{R}^{2}$.

Definition 4.5. Let $\mathcal{F}$ be a non-singular oriented foliation on $\mathbb{R}^{2}$. Let $\gamma_{1}: J_{1} \rightarrow \mathbb{R}^{2}$ and $\gamma_{2}: J_{2} \rightarrow \mathbb{R}^{2}$ be two $\mathcal{F}$-transverse paths such that $\phi_{\gamma_{1}\left(t_{1}\right)}=\phi_{\gamma_{2}\left(t_{2}\right)}=\phi$ for some $t_{1} \in J_{1}$ 
and $t_{2} \in J_{2}$. We will say that $\gamma_{1}$ and $\gamma_{2}$ has a positively $\mathcal{F}$-transverse intersection $n^{1}$ at $\phi$ if there exist $a_{1}, b_{1} \in J_{1}$ satisfying $a_{1}<t_{1}<b_{1}$ and $a_{2}, b_{2} \in J_{2}$ satisfying $a_{2}<t_{2}<b_{2}$, such that:

1. $\phi_{\gamma_{2}\left(a_{2}\right)}$ is below $\phi_{\gamma_{1}\left(a_{1}\right)}$ relative to $\phi$;

2. $\phi_{\gamma_{2}\left(b_{2}\right)}$ is above $\phi_{\gamma_{1}\left(b_{1}\right)}$ relative to $\phi$.

See Figure 4.3

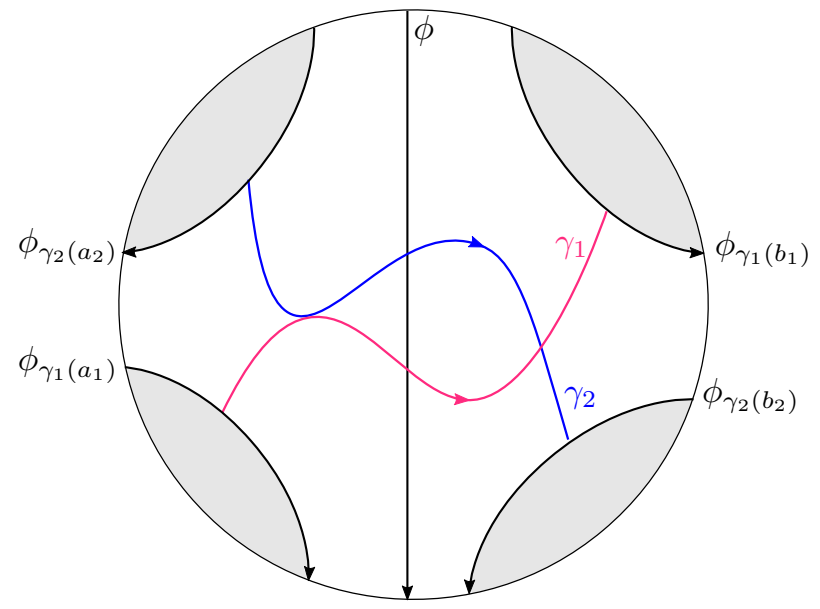

Figure 4.3: $\gamma_{1}$ intersects $\mathcal{F}$-transversally $\gamma_{2}$.

As noted in [LCT18a], if $\gamma_{1}$ intersects $\mathcal{F}$-transversally $\gamma_{2}$ and $\gamma_{1}^{\prime}, \gamma_{2}^{\prime}$ are equivalent to $\gamma_{1}$ and $\gamma_{2}$ respectively, then $\gamma_{1}^{\prime}$ intersects $\mathcal{F}$-transversally $\gamma_{2}^{\prime}$. Furthermore, if $\gamma_{1}$ intersects $\mathcal{F}$-transversally $\gamma_{2}$ then they have at least one point of intersection and so we can find two $\mathcal{F}$-transverse path $\gamma_{1}^{\prime}$ and $\gamma_{2}^{\prime}$ equivalent to $\gamma_{1}$ and $\gamma_{2}$, respectively, such that $\gamma_{1}^{\prime}$ and $\gamma_{2}^{\prime}$ have a unique intersection point $\gamma_{1}^{\prime}\left(t_{1}\right)=\gamma_{2}^{\prime}\left(t_{2}\right)$ at $\phi$. Therefore, up to equivalence, we can say that $\gamma_{1}$ and $\gamma_{2}$ has an $\mathcal{F}$-transverse intersection at $\gamma_{1}\left(t_{1}\right)=\gamma_{2}\left(t_{2}\right)$.

Let us return now to the general case of a singular oriented foliation $\mathcal{F}$ on an oriented surface $S$. Let $\gamma_{1}: J_{1} \rightarrow \operatorname{dom}(\mathcal{F})$ and $\gamma_{2}: J_{2} \rightarrow \operatorname{dom}(\mathcal{F})$ be two $\mathcal{F}$-transverse paths that meet a common leaf $\phi$. We will say that $\gamma_{1}$ and $\gamma_{2}$ have an $\mathcal{F}$-transverse intersection in $\phi$ if there exists paths $\tilde{\gamma}_{1}: J_{1} \rightarrow \widetilde{\operatorname{dom}}(\mathcal{F})$ e $\tilde{\gamma}_{2}: J_{2} \rightarrow \widetilde{\operatorname{dom}}(\mathcal{F})$ lifting $\gamma_{1}$ and $\gamma_{2}$ to $\widetilde{\operatorname{dom}}(\mathcal{F})$, respectively, with an $\tilde{\mathcal{F}}$-transverse intersection in $\tilde{\phi}$ leaf that lifts $\phi$.

When $\gamma_{1}=\gamma_{2}$ we will say that $\gamma_{1}$ has an $\mathcal{F}$-transverse self-intersection. Namely, an $\mathcal{F}$ transverse path $\gamma$ has an $\mathcal{F}$-transverse self-intersection if for every lift $\tilde{\gamma}$ to the universal covering space $\widetilde{\operatorname{dom}}(\mathcal{F})$ of $\operatorname{dom}(\mathcal{F})$, there exists a non trivial covering automorphism $T \in \operatorname{Deck}(\operatorname{dom}(\mathcal{F}))$ such that $\tilde{\gamma}$ and $T(\tilde{\gamma})$ have an $\tilde{\mathcal{F}}$-transverse intersection.

A sketch for a better understanding of an $\mathcal{F}$-transverse self-intersection is given in Figure 4.4. The surface is $\mathbb{S}^{2}$, the singular oriented foliation $\mathcal{F}$ is such that $\operatorname{sing}(\mathcal{F})=$

\footnotetext{
${ }^{1}$ As before, in the whole text "F $\mathcal{F}$-transverse intersection" will mean "positively $\mathcal{F}$-transverse intersection".
} 
$\{N, O, S\}$ and some of the leaves of $\mathcal{F}$ are represented with the colors gray, green and blue. And the $\mathcal{F}$-transverse path $\gamma$ is represented with the color pink. The paths $\tilde{\gamma}$ and $T \tilde{\gamma}$ are lifts of $\gamma$ to $\widetilde{\operatorname{dom}}(\mathcal{F})$ and they have an $\tilde{\mathcal{F}}$-transverse intersection.
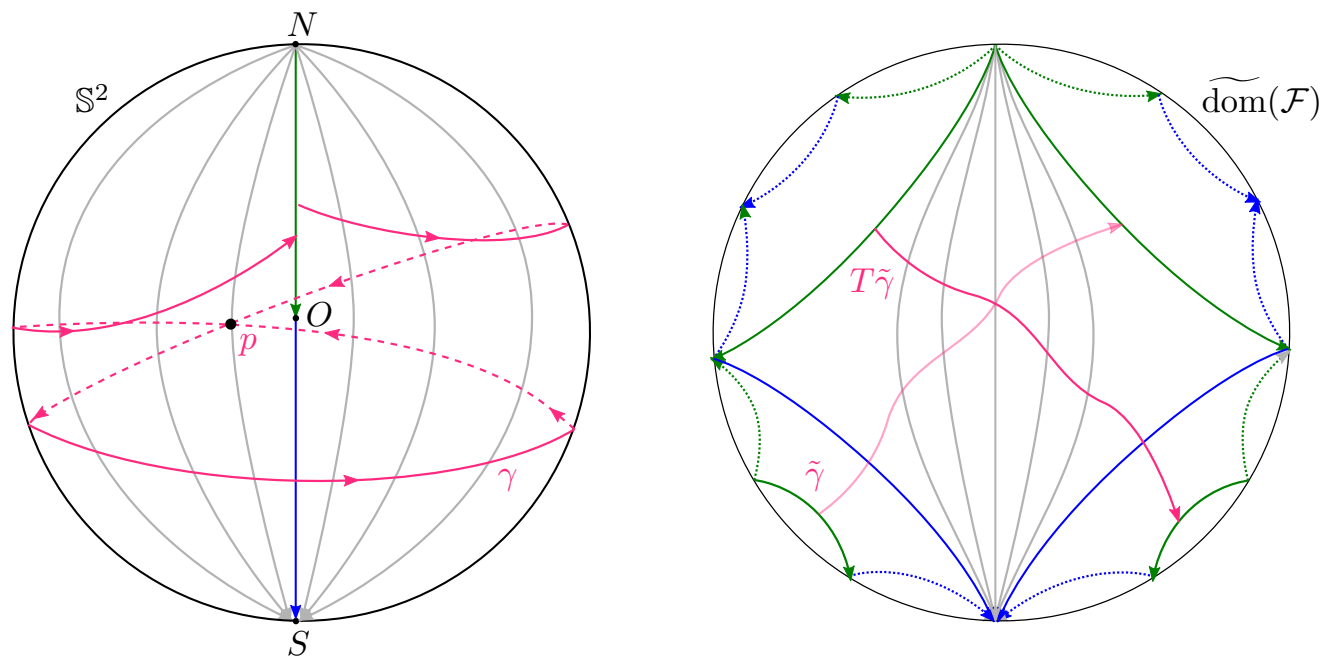

Figure 4.4: $\gamma$ has an $\mathcal{F}$-transverse self-intersection.

Let $\Gamma$ be an $\mathcal{F}$-transverse loop and $\gamma$ its natural lift. If $\gamma$ intersects $\mathcal{F}$-transversally an $\mathcal{F}$-transverse path $\gamma^{\prime}$ at a leaf $\phi$ we will say that $\Gamma$ and $\gamma^{\prime}$ has an $\mathcal{F}$-transverse intersection at $\phi$. If $\gamma^{\prime}$ is a natural lift of an $\mathcal{F}$-transverse loop $\Gamma^{\prime}$ then we will say that $\Gamma$ and $\Gamma^{\prime}$ has an $\mathcal{F}$-transverse intersection at $\phi$. Also we can define $\mathcal{F}$-transverse self-intersection for an $\mathcal{F}$-transverse loop by its natural lift like before.

Before finishing this section, we will present some results that involve the definitions presented so far and that will be useful in the rest of this text.

The first one is Proposition 1 in section 3.4 of [LCT18a] and it will be useful to prove that the set of leaves that cross a simple loop homotopic to zero in the 2-torus is an inessential set (see Definition 5.1).

Proposition 4.6. Let $\mathcal{F}$ be a singular oriented foliation on a surface and $\left(\Gamma_{i}\right)_{1 \leq i \leq m}$ a family of prime $\mathcal{F}$-transverse loops that are not pairwise equivalent. We suppose that the leaves met by the loops $\Gamma_{i}$ are never closed and that there exists an integer $N$ such that no loop $\Gamma_{i}$ meets a leaf more than $N$ times. Then, for every $i \in\{1, \cdots, m\}$, there exists an $\mathcal{F}$-transverse loop $\Gamma_{i}^{\prime}$ equivalent to $\Gamma_{i}$ such that:

1. $\Gamma_{i}^{\prime}$ and $\Gamma_{j}^{\prime}$ do not intersect if $\Gamma_{i}$ and $\Gamma_{j}$ have no $\mathcal{F}$-transverse intersection;

2. $\Gamma_{i}^{\prime}$ is simple if $\Gamma_{i}$ has no $\mathcal{F}$-transverse self-intersection.

Remark 4.7. An $\mathcal{F}$-transverse simple loop is prime. And, moreover, if $\mathcal{F}$ is a singular oriented foliation on an oriented surface $S$ that has genus 0 then, by the Jordan Curve Theorem, an $\mathcal{F}$-transverse simple loop meats a leaf of $\mathcal{F}$ just one time and, consequently, the leaves met by it are never closed. 
The following result is Proposition 2 in LCT18a and is an adapted version of the Poincaré-Bendixson Theorem. It states that an $\mathcal{F}$-bi-recurrent path on $\mathbb{S}^{2}$ has no $\mathcal{F}$ transverse self-intersection if and only if it is equivalent to the natural lift of a simple loop. Moreover, the set of the leaves crossed by it is an open annulus.

Proposition 4.8. Let $\mathcal{F}$ be a singular oriented foliation on $\mathbb{S}^{2}$ and $\gamma: \mathbb{R} \rightarrow \mathbb{S}^{2}$ an $\mathcal{F}$-bi-recurrent transverse path. The following properties are equivalent:

1. $\gamma$ has no $\mathcal{F}$-transverse self-intersection;

2. There exists an $\mathcal{F}$-transverse simple loop $\Gamma_{0}$ such that $\gamma$ is equivalent to the natural lift $\gamma_{0}$ of $\Gamma_{0}$, and

3. The set $U=\bigcup_{t \in \mathbb{R}} \phi_{\gamma(t)}$ is an open annulus.

As a scholium of the proof of this proposition we have that an $\mathcal{F}$-transverse loop $\Gamma$ with no $\mathcal{F}$-transverse self-intersection is equivalent to a multiple of an $\mathcal{F}$-transverse simple loop $\Gamma_{0}$.

Definition 4.9 (Section 4.1 in [LCT18b]). Let $\mathcal{F}$ be a singular oriented foliation on $\mathbb{S}^{2}$. Let $\gamma: J \rightarrow \operatorname{dom}(\mathcal{F})$ be an $\mathcal{F}$-transverse path and $\Gamma_{0}: \mathbb{T}^{1} \rightarrow \operatorname{dom}(\mathcal{F})$ be an $\mathcal{F}$-transverse loop. We will say that $\gamma$ draws $\Gamma_{0}$ if there exist $a<b$ in $J$ and $t \in \mathbb{R}$ such that $\left.\gamma\right|_{[a, b]}$ is $\mathcal{F}$-equivalent to $\left.\gamma_{0}\right|_{[t, t+1]}$, where $\gamma_{0}$ is a natural lift of $\Gamma_{0}$.

Let $\gamma: J \rightarrow \operatorname{dom}(\mathcal{F})$ be an $\mathcal{F}$-transverse path that draws a simple loop $\Gamma_{0}$ and let $U_{\Gamma_{0}}$ be the open annulus of leaves that are crossed by $\Gamma_{0}$. We have the set

$$
J_{\Gamma_{0}}^{\gamma}=\left\{t \in J \mid \gamma(t) \in U_{\Gamma_{0}}\right\}
$$

We will say that a connected component $J_{0} \in J_{\Gamma_{0}}^{\gamma}$ is a drawing component of $\gamma$ if $\left.\gamma\right|_{J_{0}}$ draws $\Gamma_{0}$. We will also define a crossing component of $\gamma$ as a connected component $J_{1} \in J_{\Gamma_{0}}^{\gamma}$ such that both ends $a$ and $b$ of $J_{1}$ are in $J$ and $\gamma(a)$ and $\gamma(b)$ are in different components of $\mathbb{S}^{2} \backslash U_{\Gamma_{0}}$. In this case, we will say that $\left.\gamma\right|_{J_{1}}$ crosses $\Gamma_{0}$.

To finish this section, the last result is Proposition 24 in section 4.2 of [LCT18b]. It is a good tool for determining when a path on $\mathbb{S}^{2}$ has an $\mathcal{F}$-transverse self-intersection.

Proposition 4.10. Let $\mathcal{F}$ be a singular oriented foliation on sphere $\mathbb{S}^{2}$ and suppose that $\gamma: J \rightarrow \operatorname{dom}(\mathcal{F})$ is an $\mathcal{F}$-transverse path with no $\mathcal{F}$-transverse self-intersection, then

1. If $\gamma$ draws an $\mathcal{F}$-transverse simple loop $\Gamma_{0}$, there exists a unique drawing component of $J_{\Gamma_{0}}^{\gamma}$;

2. If $\gamma$ draws and crosses an $\mathcal{F}$-transverse simple loop $\Gamma_{0}$, there exists a unique crossing component of $J_{\Gamma_{0}}^{\gamma}$ and it coincides with the drawing component; 
3. if $\gamma$ draws and does not crosses an $\mathcal{F}$-transverse simple loop $\Gamma_{0}$, the drawing component of $J_{\Gamma_{0}}^{\gamma}$ coincides with $J$ in a neighborhood of at least one endpoint of $J$.

In what has been discussed so far, we have not introduced any surface homeomorphisms.To make this, we will use Brouwer-Le Calvez Foliation Theory (see [Bro12], [Fra92, [LC04] and [LC05]) and the concept of Maximal Isotopy whose existence was proved by Béguin, Crovisier and Le Roux in [BCLR20]. These are the content of the next sections.

\subsection{Brouwer - Le Calvez Foliation}

This section is dedicated to presenting Brouwer's Theory and the famous Brouwer Translation Theorem due to L. E. J. Brouwer, see [Bro12] (or [Fra92]). In addition, at the end, we will present the Equivariant Foliation Theorem, due to Le Calvez (see [LC04] and [LC05]), that is the link between Brouwer Theory and surfaces homeomorphisms.

Definition 4.11. A homeomorphism $\check{f}: \mathbb{R}^{2} \rightarrow \mathbb{R}^{2}$ of the plane that preserves orientation and is fixed point free is called a Brouwer's homeomorphism.

There is no recurrence in a Brouwer's homeomorphism, indeed we have the following:

Lemma 4.12. If $\check{f}: \mathbb{R}^{2} \rightarrow \mathbb{R}^{2}$ is a Brouwer's homeomorphism then all points $\check{z} \in \mathbb{R}^{2}$ are wandering.

The next result is the Brouwer's Translation Theorem, which is one of the basic results for what will come next.

Theorem 4.13 (Brouwer's Translation Theorem). Let $\check{f}: \mathbb{R}^{2} \rightarrow \mathbb{R}^{2}$ be a Brouwer homeomorphism and let $\check{z} \in \mathbb{R}^{2}$, then there exists a line $\check{\phi}: \mathbb{R} \rightarrow \mathbb{R}^{2}$, with $\check{\phi}(0)=\check{z}$ such that

$$
\check{f}(\check{\phi}) \subset L(\check{\phi}) \text { e } \check{f}^{-1}(\check{\phi}) \subset R(\check{\phi})
$$

The line $\check{\phi}$ that satisfies the above conclusion is called a Brouwer line for $\check{f}$. Note that if $\check{\phi}: \mathbb{R} \rightarrow \mathbb{R}^{2}$ is a Brouwer line for $\check{f}$ then $\check{f}(\overline{L(\check{\phi})}) \subset L(\check{\phi})$ and $\check{f}^{-1}(\overline{R(\check{\phi})}) \subset R(\check{\phi})$.

The Brouwer Translation Theorem asserts that $\mathbb{R}^{2}$ can be covered by Brouwer lines in case that $\check{f}$ is a Brouwer homeomorphism. Le Calvez, in [LC04] and [LC05] extended this result showing that it is possible to obtain a non-singular oriented foliation on the plane by Brouwer lines for a Brouwer's homeomorphism $\check{f}$.

Theorem 4.14 (Le-Calvez, see [LC04]). Let $\check{f}: \mathbb{R}^{2} \rightarrow \mathbb{R}^{2}$ be a Brouwer homeomorphism, then there exist a non-singular oriented foliation $\check{\mathcal{F}}$ of $\mathbb{R}^{2}$ whose leaves are Brouwer lines for $\check{f}$. 
Remark 4.15. Note that Theorem 4.14 establishes that the dynamics of the foliation is, in some sense, transverse to the homeomorphism $\check{f}$, which is a dynamically relevant result. This means that given $\check{z}_{0} \in \mathbb{R}^{2}$ it is possible to construct a path $\check{\gamma}:[a, b] \rightarrow \mathbb{R}^{2}$ with $\check{\gamma}(a)=\check{z}_{0}$ e $\check{\gamma}(b)=\check{f}\left(\check{z}_{0}\right)$ and so that the leaves of $\check{\mathcal{F}}$ crossed by $\check{\gamma}$ are crossed from right to left, that is $\check{\gamma}$ is positively transverse to $\check{\mathcal{F}}$.

In fact, if $W$ is the set of points $\check{z} \in \mathbb{R}^{2}$ that are joined to $\check{z}_{0}$ by a positively $\check{\mathcal{F}}$-transverse path then $W$ is an open set whose boundary consists of the leaf $\check{\phi}_{\check{z}_{0}}$ that contains $\check{z}_{0}$ and, eventually, some other leaves $\check{\phi}$ such that $W \subset L(\check{\phi})$, see figure 4.5 . If $\check{f}\left(\check{z}_{0}\right)$ did not belong to $W$ then there is a leaf $\check{\phi} \in \partial W$ that $\check{f}\left(\check{z}_{0}\right) \in \overline{R(\check{\phi})}$ and $\check{z}_{0} \in L(\check{\phi})$, but this is a contradiction with the fact that $\check{\phi}$ is a Brouwer line, that is $\check{f}^{-1}(\overline{R(\check{\phi})}) \subset R(\check{\phi})$.

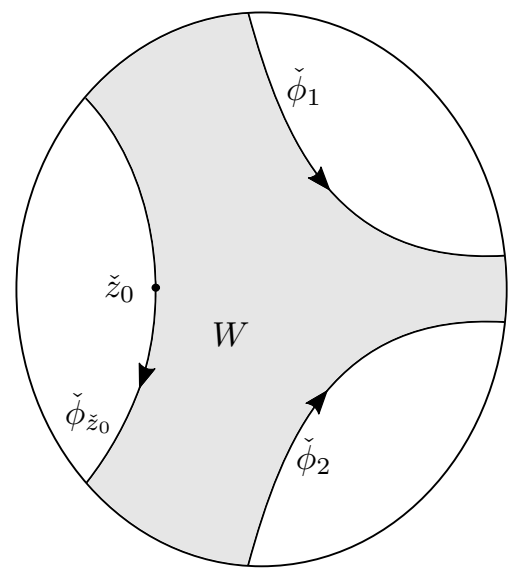

Figure 4.5: $\check{\phi}_{\check{z}_{0}}, \check{\phi}_{1}, \check{\phi}_{2} \in \partial W$.

The equivariant version of Theorem 4.14 is also due to Le-Calvez (see Theorem 1.2 in [LC05]). The author showed that if a Brouwer's homeomorphism commutes with the transformations of a group $G$ of homeomorphisms acting freely and properly on the plane (see Definition 2.7) then in addition to the foliation being made of Brouwer lines, it is invariant by the action of $G$ on $\mathbb{R}^{2}$. The statement is the following:

Theorem 4.16 (Equivariant Foliation Theorem). Let $\check{f}: \mathbb{R}^{2} \rightarrow \mathbb{R}^{2}$ be a Brouwer homeomorphism and $G$ a discrete group of homeomorphisms of the plane that preserve orientation, which acts freely and properly on $\mathbb{R}^{2}$. If $\check{f}$ commutes with the elements of $G$ then there is a foliation $\check{\mathcal{F}}$ by Brouwer lines for $\check{f}$ which is invariant for $G$.

Now, let $S$ be a oriented surface and let $f \in \operatorname{Homeo}_{0}(S)$. A point $z \in \operatorname{fix}(f)$ is said to be a contractible fixed point for $I$ if its trajectory along $I$ is a loop homotopic to zero on $S$. In fact, it is possible to show that a contractible fixed point point for $I$ is a fixed point for the lift $\breve{f}=\breve{f}_{1}$ of $f$ associated to $I$ to the universal covering space $\breve{S}$ of $S$.

With this definition, we have the following consequence of Theorem 4.16 , that follows immediately from Remark 4.15 .

Corollary 4.17. Let $S$ be a surface, $I=\left(f_{t}\right)_{t \in[0,1]}$ an identity isotopy on $S$ and let $f:=f_{1}$. Suppose that I does not have a contractible fixed point, then there exists a non-singular 
oriented foliation $\mathcal{F}$ on $S$ such that for all point $z \in S$ there is a positively $\mathcal{F}$-transverse path $\gamma$ that joins $z$ to $f(z)$ and is homotopic to $I(z)$, with the endpoints fixed.

Proof. Let $\breve{I}=\left(\breve{f}_{t}\right)_{t \in[0,1]}$ be the identity isotopy that lifts $I$ to the universal covering space $\breve{S}$ of $S$, let $\breve{f}:=\breve{f}_{1}$. Then, because of the absence of contractible fixed points for $I, \breve{f}$ has no fixed point, which implies that each connected component of $\breve{S}$ is homeomorphic to the plane.

Therefore, restrict to each connect component $S_{i},\left.\breve{f}\right|_{S_{i}}$ is a Brouwer homeomorphism and considering $\operatorname{Deck}(S)$ as the discrete group of the covering automorphism, there exists a non-singular oriented foliation $\breve{\mathcal{F}}$ of $S$ by Brouwer lines that is invariant by $\operatorname{Deck}(S)$.

And by Remark 4.15 if we take any point $\breve{z} \in \breve{\pi}^{-1}(z)$ then there is a positively $\breve{\mathcal{F}}$ transverse path $\breve{\gamma}$ joining $\breve{z}$ to $\breve{f}(\breve{z})$ that must be homotopic to $\breve{I}(\breve{z})$. And finally, as $\breve{\mathcal{F}}$ is invariant by $\operatorname{Deck}(S)$, if we take the projections of $\breve{\mathcal{F}}$ and $\breve{\gamma}$, then we conclude the proof.

Corollary 4.17 has a very strong hypothesis: the identity isotopy $I$ cannot have contractible fixed points. Some works (see [Jau14] and [BCLR20], for example) have emerged to provide a solution to this problem.

In Jau14, Jaulent proved that given an $f \in \operatorname{Homeo}_{0}(S)$, there exists a closed subset $F \subset \operatorname{fix}(f)$ with the following property: in $S \backslash F$ there exists an isotopy from the identity to the restriction $\left.f\right|_{S \backslash F}$ of $f$, such that no trajectory for this isotopy is a contractible loop. And this result is sufficient for the most applications of Theorem 4.17, and indeed, Le Calvez and Tal, use this formalism in [LCT18a].

But recently, Beguin, Crovisier and Le Roux (see [BCLR20]) presented a stronger tool that improves Jaulent's result. The authors find a closed subset $F$ with the same properties as prescribed in the previous paragraph, but they furthermore get an, identity isotopy $I=\left(f_{t}\right)_{t \in[0,1]}$ in $S \backslash F$ that extends continuously to the identity in $F$. So, it is an identity isotopy for a homeomorphism $f: S \rightarrow S$ such that for every point $z \in F$ we have that $f_{t}(z)=z$.

In the following subsection we will present and discuss how we can apply Corollary 4.17 for the homeomorphism $f: S \rightarrow S$ using the result due to Beguin, Crovisier and Le Roux ([BCLR20]).

\subsubsection{Maximal Isotopy and Transverse Foliations}

Definition 4.18. Let $f \in \operatorname{Homeo}(S)$ be isotopic to identity on the oriented surface $S$ and let $I \in \mathcal{I}_{f}$ be such an isotopy. We define the fixed point set of $I$ as the set

$$
\operatorname{fix}(I)=\bigcap_{t \in[0,1]} \operatorname{fix}\left(f_{t}\right)
$$

If $z \in \operatorname{fix}(I)$ then $z$ is called fixed point for the isotopy $I$. 
The complement set of fix $(I)$ in $S, S \backslash$ fix $(I)$ will be called domain of $I$ and will be denoted by $\operatorname{dom}(I)$. Note that $\operatorname{fix}(I)$ is a closed set of $S$, so $\operatorname{dom}(I)$ is itself an oriented surface. Furthermore, the restriction $\left.f\right|_{\operatorname{dom}(I)}$ is a homeomorphism isotopic to identity on $\operatorname{dom}(I)$, where $\left.I\right|_{\operatorname{dom}(I)}$ is an identity isotopy from Id $\left.\right|_{\operatorname{dom}(I)}$ to $\left.f\right|_{\operatorname{dom}(I)}$.

We can define a partial order on $\mathcal{I}_{f}$ as follows: given $I^{\prime}$ and $I$ two identity isotopies of $f$, we say that $I^{\prime} \preceq I$ if

1. $\operatorname{fix}\left(I^{\prime}\right) \subset \operatorname{fix}(I)$ and

2. $I$ is homotopic to $I^{\prime}$ relative to fix $\left(I^{\prime}\right)$.

Thus, we say that $I$ is a maximal isotopy of $f$, if it is maximal for the partial order above.

Lemma 4.19 (see Lemma A.8 in [BCLR20]). Let $S$ be a connected surface, $f: S \rightarrow S$ be a homeomorphism isotopic to identity and $I^{\prime} \in \mathcal{I}_{f}$. If $z \in \operatorname{fix}(f)$ is a contractible fixed point for $I^{\prime}$ then there is another isotopy $I \in \mathcal{I}_{f}$ such that $z \in \operatorname{fix}(I)$.

A consequence of Lemma 4.19 and the partial order above is the following:

Corollary 4.20. If $I \in \mathcal{I}$ is maximal then $\left.f\right|_{\operatorname{dom}(I)}$ has no contractible fixed point for $I$.

So, it becomes reasonable to ask: given a homeomorphism $f$ isotopic to identity and an identity isotopy $I^{\prime}$, is there a maximal isotopy $I$ of $f$, such that $I^{\prime} \preceq I$ ? The answer for this question was given by Béguin, Crovisier and Le Roux in [BCLR20]:

Theorem 4.21 (Maximal Isotopy, see Corollary 1.3 in [BCLR20]). Let $f: S \rightarrow S$ be a homeomorphism isotopic to identity, then for every $I^{\prime} \in \mathcal{I}_{f}$, there exists $I \in \mathcal{I}_{f}$ such that $I^{\prime} \preceq I$ and $I$ is maximal for the partial order above.

Let us clarify the use of maximal isotopies to apply Theorem 4.17 .

Let $f: S \rightarrow S$ be a homeomorphism isotopic to identity on a surface $S$. Let $I^{\prime} \in \mathcal{I}_{f}$ be an identity isotopy of $f$. Then by Theorem 4.21 there is a maximal isotopy $I \in \mathcal{I}_{f}$ such that $I^{\prime} \preceq I$. Then, by Corollary $4.20,\left.f\right|_{\operatorname{dom}(I)}$ the restriction of $f$ to the domain of $I$ has no contractible fixed points. And then by Theorem 4.17 there exist a singular oriented foliation $\mathcal{F}$ on $S$ such that $\operatorname{sing}(\mathcal{F})=\operatorname{fix}(I)$ (therefore, $\operatorname{dom}(\mathcal{F})=\operatorname{dom}(I)$ ) and for all point $z \in \operatorname{dom}(I)$ there is a positively $\mathcal{F}$-transverse path $\gamma$ that joint $z$ to $f(z)$ and is homotopic to $I(z)$, with the endpoints fixed.

The foliation $\mathcal{F}$ that satisfy the above conclusion is said transverse to the isotopy $I$.

\subsection{Transverse Trajectories}

Until the end of this section, we will relate the definitions in section 4.1 with the dynamics. 
Let $f \in \operatorname{Homeo}(S)$ be isotopic to the identity. Then given an identity isotopy $I^{\prime} \in \mathcal{I}_{f}$, let $I \in \mathcal{I}_{f}$ be such that $I^{\prime} \preceq I$ and $I$ is a maximal isotopy of $f$. Let $\mathcal{F}$ be a transverse foliation to $I$. We will fix the following notation:

Notation 4.22. Given a maximal isotopy $I$ of $f$, let $\widetilde{\operatorname{dom}}(I)$ be the universal covering of $\operatorname{dom}(I), \tilde{\pi}: \widetilde{\operatorname{dom}}(I) \rightarrow \operatorname{dom}(I)$ be the universal covering projection, $\tilde{f}$ be the lift of $\left.f\right|_{\operatorname{dom}(I)}$, induced by identity isotopy $\tilde{I}=\left(\tilde{f}_{t}\right)_{t \in[0,1]}$ that lifts $\left.I\right|_{\operatorname{dom}(I)}$ to the universal covering space $\widetilde{\operatorname{dom}}(I)$. We will write $\tilde{\mathcal{F}}$ for the lifted foliation on $\widetilde{\operatorname{dom}}(I)$ from $\left.\mathcal{F}\right|_{\operatorname{dom}(I)}$.

From the equivalence class on Definition 4.2 , we have the $\mathcal{F}$-transverse path $\gamma$ given by Theorem 4.17 is uniquely defined up to equivalence.

We will write $I_{\mathcal{F}}(z)$ for the class of the paths $\gamma:[a, b] \rightarrow \operatorname{dom}(I)$ that is $\mathcal{F}$-transverse and homotopic to $I(z)$, relative to the endpoints. We will also use de notation $I_{\mathcal{F}}(z)$ for any representative in this class and call it the transverse trajectory of $z$. As before, we can define by concatenation $I_{\mathcal{F}}^{n}(z)=\prod_{0 \leq k<n} I_{\mathcal{F}}\left(f^{k}(z)\right)$ for every integer $n \geq 1$. Similarly we define

$$
I_{\mathcal{F}}^{\mathbb{N}}(z)=\prod_{k \geq 0} I_{\mathcal{F}}\left(f^{k}(z)\right), I_{\mathcal{F}}^{-\mathbb{N}}(z)=\prod_{k \leq 0} I_{\mathcal{F}}\left(f^{k}(z)\right), I_{\mathcal{F}}^{\mathbb{Z}}(x)=\prod_{k \in \mathbb{Z}} I_{\mathcal{F}}\left(f^{k}(z)\right)
$$

and the last one will be called the whole transverse trajectory of the point $z \in S$.

Note that if $z \in \operatorname{dom}(I)$ is a periodic point of period $q \geq 1$ then its transverse trajectory until order $q$ define an $\mathcal{F}$-transverse loop $\Gamma$ whose the natural lift $\gamma$ satisfies the property that $\left.\gamma\right|_{[0,1]}=I_{\mathcal{F}}^{q}(z)$.

The following lemma is a result that will be very useful in Chapter 5 .

Lemma 4.23 (Lemma 17 of [LCT18a]). Let $z \in \operatorname{dom}(I)$, take any integer $n \geq 1$.

1. There exists a neighborhood $W$ of $z$ such that, for every $z^{\prime}, z^{\prime \prime} \in W$, the path $I_{\mathcal{F}}^{n}\left(z^{\prime}\right)$ is $\mathcal{F}$-equivalent to a subpath of $I_{\mathcal{F}}^{n+2}\left(f^{-1}\left(z^{\prime \prime}\right)\right)$. See Figure 4.6 .

2. For every $z^{\prime} \in \omega_{f}(z)$ (respectively $z^{\prime} \in \alpha_{f}(z)$ ) and every $m \geq 0$, the path $I_{\mathcal{F}}^{n}\left(z^{\prime}\right)$ is $\mathcal{F}$-equivalent to a subpath of $I_{\mathcal{F}}^{\mathbb{N}}\left(f^{m}(z)\right)$ (respectively $I_{\mathcal{F}}^{-\mathbb{N}}\left(f^{-m}(z)\right)$ ).

Indeed, item (2.) of the above lemma follows from item (1.). Just observe that given $m \geq 0$, as $z^{\prime} \in \omega_{f}(z)$, there is some integer $m_{k}>m$ such that $f^{m_{k}}(z) \in W$, where $W$ is the neighborhood given by item (1.).

It follows from the above lemma and from Proposition 4.10 the consequence below:

Corollary 4.24 (Proposition 27 in [LCT18b]). Let $f$ be an orientation preserving homeomorphism of $\mathbb{S}^{2}$ with no topological horseshoe. Let $I$ be a maximal isotopy of $f$ and $\mathcal{F}$ a transverse foliation to I. If $z \in \operatorname{dom}(I)$ is a non-wandering point of $f$, then:

1. Either $I_{\mathcal{F}}^{\mathbb{Z}}(z)$ never meets a leaf twice; 


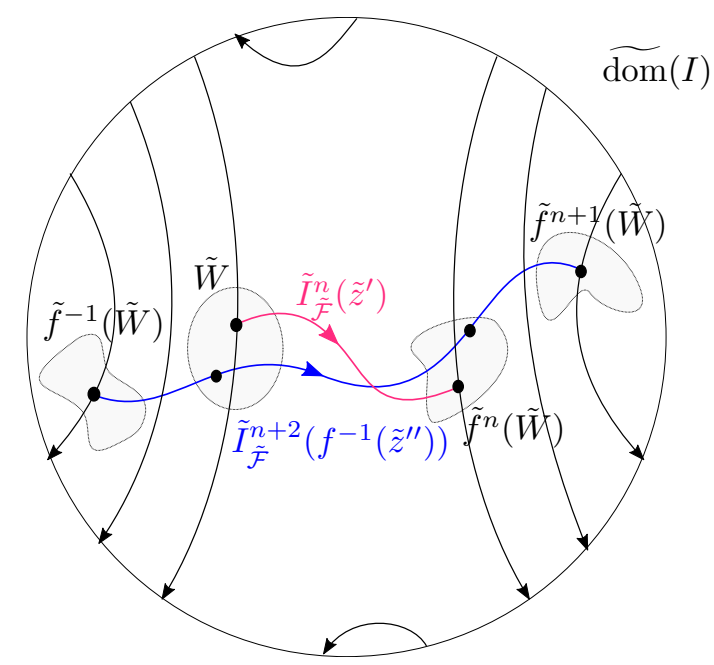

Figure 4.6: $\tilde{I}_{\tilde{\mathcal{F}}}^{n}\left(\tilde{z}^{\prime}\right)$ is $\tilde{\mathcal{F}}$-equivalent $\tilde{I}_{\tilde{\mathcal{F}}}^{n+2}\left(\tilde{f}^{-1}\left(\tilde{z}^{\prime \prime}\right)\right)$ in $\widetilde{\operatorname{dom}}(I)$.

2. $\operatorname{Or} I_{\mathcal{F}}^{\mathbb{Z}}(z)$ is equivalent to an $\mathcal{F}$-transverse simple loop $\Gamma_{0}$.

As a scholium of the proof of this corollary we have that if $f$ is an orientation preserving homeomorphism of $\mathbb{S}^{2}, I$ a maximal isotopy of $f$ and $\mathcal{F}$ a transverse foliation to $I$ and the whole transverse trajectory of a non-wandering point $z \in \operatorname{dom}(I)$ of $f$ has no $\mathcal{F}$-transverse self-intersection then also the conclusion of the corollary is true: either item (1.) or item (2.) hold.

It is worth noting that the first case happens only when $\alpha(z)$ and $\omega(z)$ are included in fix $(I)$, see the remark after Proposition 27 in [LCT18b].

\section{Admissible paths}

Note that the transverse trajectory $I_{\mathcal{F}}(z)$ of a point $z \in \operatorname{dom}(I)$ needs to start at the point $z$ and end at the point $f(z)$. The next definition removes this requirement.

Definition 4.25. An $\mathcal{F}$-transverse path $\gamma:[a, b] \rightarrow \operatorname{dom}(I)$ is admissible of order $n$ (or $n$-admissible) if it is equivalent to a path $I_{\mathcal{F}}^{n}(z)$ for some $z \in \operatorname{dom}(I)$.

This definition is equivalent to the following property: there exists a lift $\tilde{\gamma}:[a, b] \rightarrow$ $\widetilde{\operatorname{dom}}(I)$ of $\gamma$ and a point $\tilde{z} \in \widetilde{\operatorname{dom}}(I)$ such that $\tilde{z} \in \tilde{\phi}_{\tilde{\gamma}(a)}$ and $\tilde{f}^{n}(\tilde{z}) \in \tilde{\phi}_{\tilde{\gamma}(b)}$, that is,

$$
\tilde{f}^{n}\left(\tilde{\phi}_{\tilde{\gamma}(a)}\right) \cap \tilde{\phi}_{\tilde{\gamma}(b)} \neq \emptyset
$$

We will say that an $\mathcal{F}$-transverse path $\gamma:[a, b] \rightarrow \operatorname{dom}(I)$ is admissible of order $\leq n$ if it is a sub-path of an admissible path of order $n$.

More generally, we will say that an $\mathcal{F}$-transverse path $\gamma: J \rightarrow \operatorname{dom}(I)$ defined on any real interval is admissible if for every interval $[a, b] \subset J$, there exists $n \geq 1$ such that $\left.\gamma\right|_{[a, b]}$ is admissible of order $\leq n$.

We will say that an $\mathcal{F}$-transverse loop $\Gamma: \mathbb{T}^{1} \rightarrow \operatorname{dom}(I)$ is $n$-admissible if for every positive integer $k$ the natural lift $\gamma$ of $\Gamma$ restricted to the interval $[0, k] \in \mathbb{R},\left.\gamma\right|_{[0, k]}$ is 
$n k$-admissible. Moreover, if it is a sub-path of an admissible path of order $n k$ then it will be said $\leq n$-admissible. To finish, an $\mathcal{F}$-transverse loop $\Gamma: \mathbb{T}^{1} \rightarrow \operatorname{dom}(I)$ is admissible if for every interval $[a, b] \subset \mathbb{R}$, there exists $n \geq 1$ such that its natural lift $\gamma$ restrict to $[a, b]$ is admissible of order $\leq n$.

Note that if $z \in \operatorname{dom}(I)$ is a periodic point of period $q \geq 1$ then its whole transverse trajectory is $\mathcal{F}$-equivalent to the natural lift $\gamma$ of an $\mathcal{F}$-transverse loop $\Gamma$ that is $q$-admissible.

To finish this section we will present some results that involve transverse trajectories, admissible paths and $\mathcal{F}$-transverse (self-)intersection.

We will start with the Forcing Proposition that is the central result in forcing theory for surface homeomorphism. It establishes an operation that allows us to build admissible paths from a pair of admissible paths with an $\mathcal{F}$-transverse intersection.

Proposition 4.26 (Proposition 20, [LCT18a]). Let $S$ be an oriented surface. Suppose that $\gamma_{1}:\left[a_{1}, b_{1}\right] \rightarrow S$ and $\gamma_{2}:\left[a_{2}, b_{2}\right] \rightarrow S$ are $\mathcal{F}$-transverse paths with an $\mathcal{F}$-transverse intersection at the point $\gamma_{1}\left(t_{1}\right)=\gamma_{2}\left(t_{2}\right)$. If $\gamma_{1}$ is $n_{1}$-admissible and $\gamma_{2}$ is $n_{2}$-admissible, we have that $\left.\left.\gamma_{1}\right|_{\left[a_{1}, t_{1}\right]} \gamma_{2}\right|_{\left[t_{2}, b_{2}\right]}$ and $\left.\left.\gamma_{2}\right|_{\left[a_{2}, t_{2}\right]} \gamma_{1}\right|_{\left[t_{1}, b_{1}\right]}$ are $\left(n_{1}+n_{2}\right)$-admissible.

We already know, by the techniques developed in [LM91] due to Libre and Mackay, that if the rotation set of $f \in \mathrm{Homeo}_{0}\left(\mathbb{T}^{2}\right)$ has a not empty interior then $f$ has a topological horseshoe. But we can also prove this result using forcing theory for transverse trajectories. The following result helps us to justify that.

Lemma 4.27 (Lemma 30, [LCT18a]). Let $\gamma_{1}: \mathbb{R} \rightarrow \operatorname{dom}(I)$ and $\gamma_{2}: \mathbb{R} \rightarrow \operatorname{dom}(I)$ be two admissible $\mathcal{F}$-transverse recurrent paths (possibly equal) with an $\mathcal{F}$-transverse intersection. Then there exists an admissible $\mathcal{F}$-transverse loop $\Gamma$ with a $\mathcal{F}$-transverse self-intersection.

The next and last statement is a fundamental theorem of [LCT18b], which is a purely topological criterion for the existence of a topological horseshoe stated in terms of transverse trajectories.

Theorem 4.28 (Topological Horseshoe, Theorem N, [LCT18b]). Let $S$ be an oriented surface, $f$ a homeomorphism isotopic to identiy on $S, I$ a maximal isotopy of $f$ and $\mathcal{F}$ a transverse foliation to $I$. If there exists a point $z$ in the $\operatorname{dom}(I)$ and an integer $r \geq 1$ such that the transverse trajectory $I_{\mathcal{F}}^{r}(z)$ has an $\mathcal{F}$-transverse self-intersection at $I_{\mathcal{F}}^{r}(z)(s)=I_{\mathcal{F}}^{r}(z)(t)$, where $s<t$, then $f$ has a topological horseshoe. 


\section{Chapter 5}

\section{Proofs of Theorem A and Proposition C}

The purpose of this chapter is to present the proofs of Theorem A and Proposition C. We will use the same notations established in the previous chapters.

We will start with the definition of inessential, essential and totally essential sets of $\mathbb{T}^{2}$ and in sequence we will prove that the set of leaves of an $\mathcal{F}$-transverse loop homotopic to zero in $\mathbb{T}^{2}$ is an inessential set.

Definition 5.1. An open subset $U$ of $\mathbb{T}^{2}$ is inessential if every loop in $U$ is homotopic to zero in $\mathbb{T}^{2}$. Otherwise, $U$ is essential. An arbitrary set $E$ of $\mathbb{T}^{2}$ is called inessential if it has some inessential neighborhood. And, finally, $E \subset \mathbb{T}^{2}$ is fully essential if $\mathbb{T}^{2} \backslash E$ is inessential.

Observe that if $U \subset \mathbb{T}^{2}$ is open and connected then $U$ is inessential if and only if, for every $\check{U} \in \check{\pi}^{-1}(U), \check{U} \cap\left(\check{U}+\left(p_{1}, p_{2}\right)\right)=\emptyset$ for any $\left(p_{1}, p_{2}\right) \in \mathbb{Z}^{2} \backslash\{(0,0)\}$. For more details and properties of this kind of sets see [KT14].

Let $\mathcal{F}$ be a singular oriented foliation on $\mathbb{T}^{2}$. We will denote by $\check{\mathcal{F}}$ its lift to $\mathbb{R}^{2}$. As observed in the previous chapter, we have that $\operatorname{dom}(\check{\mathcal{F}})=\check{\pi}^{-1}(\operatorname{dom}(\mathcal{F}))$.

Let $\check{\Gamma}: \mathbb{T}^{1} \rightarrow \operatorname{dom}(\check{\mathcal{F}})$ be an $\check{\mathcal{F}}$-transverse simple loop on $\operatorname{dom}(\check{\mathcal{F}})$, we will denote by $\mathrm{B}_{\mathrm{c}}(\check{\Gamma})$ and $\mathrm{UB}_{\mathrm{c}}(\check{\Gamma})$ the bounded and unbounded connected componentes of $\mathbb{R}^{2} \backslash \check{\Gamma}$ respectively.

Remember that a natural lift of $\check{\Gamma}$ is a continuous map $\check{\gamma}: \mathbb{R} \rightarrow \operatorname{dom}(\check{\mathcal{F}})$ such that $\check{\gamma}(t)=\check{\gamma}(t+1)$ and lifts $\check{\Gamma}$, in the sense that if $\pi: \mathbb{R} \rightarrow \mathbb{T}^{1}$ is the canonical universal covering projection of $\mathbb{T}^{1}$ then $\check{\gamma}=\check{\Gamma} \circ \pi$.

Let $\check{U}=\bigcup_{t \in \mathbb{R}} \phi_{\check{\gamma}(t)}$ the open topological annulus of the leaves that are crossed by $\check{\Gamma}$ on $\mathbb{R}^{2}$ (see Proposition 4.8). Similarly we will denote $\mathrm{B}_{\mathrm{c}}(\check{U})$ as the bounded connected component of $\mathbb{R}^{2} \backslash \check{U}$ and $\mathrm{UB}_{\mathrm{c}}(\check{U})$ as the union of the unbouded connected components of $\mathbb{R}^{2} \backslash \check{U}$. Notice that $\mathrm{B}_{\mathrm{c}}(\check{U}) \subset \mathrm{B}_{\mathrm{c}}(\check{\Gamma})$ is a closed set because it is a connected component of the complement of an open set. So, $\mathrm{B}_{\mathrm{c}}(\check{U})$ is a compact subset of $\mathbb{R}^{2}$.

Lemma 5.2. Let $\check{\Gamma}$ be an $\mathcal{F}$-transverse simple loop on $\mathbb{R}^{2}$, $\check{\gamma}$ its natural lift and $\check{U}=$ $\bigcup_{t \in \mathbb{R}} \phi_{\check{\gamma}(t)}$ the open topological annulus of the leaves that crosses $\check{\Gamma}$ on $\mathbb{R}^{2}$. Then one of the following properties holds: 
1. $\omega(\check{\phi}) \neq \emptyset, \omega(\check{\phi}) \subset \mathrm{B}_{\mathrm{c}}(\check{U})$ and $\alpha(\check{\phi}) \not \subset \mathrm{B}_{\mathrm{c}}(\check{U})$, for all $\check{\phi} \subset \check{U}$;

2. $\alpha(\check{\phi}) \neq \emptyset, \alpha(\check{\phi}) \subset \mathrm{B}_{\mathrm{c}}(\check{U})$ and $\omega(\check{\phi}) \not \subset \mathrm{B}_{\mathrm{c}}(\check{U})$, for all $\check{\phi} \subset \check{U}$.

Proof. $\check{\phi} \subset \check{U}$ implies that $\check{\phi} \cap \check{\Gamma} \neq \emptyset$ and as $\check{\Gamma}$ is an $\mathcal{F}$-transverse simple loop we have that $\check{\phi} \cap \check{\Gamma}=\{z\}$. Then we have two possibilities that are excluding:

a) $\check{\phi}_{z}^{+} \subset \mathrm{B}_{\mathrm{c}}(\check{\Gamma})$

b) $\check{\phi}_{z}^{-} \subset \mathrm{B}_{\mathrm{c}}(\check{\Gamma})$

If item (a) holds we have $\omega(\check{\phi}) \subset \mathrm{B}_{\mathrm{c}}(\check{\Gamma}) \cup \check{\Gamma}$ and $\alpha(\check{\phi}) \not \subset \mathrm{B}_{\mathrm{c}}(\check{\Gamma}) \cup \check{\Gamma}$. As $\mathrm{B}_{\mathrm{c}}(\check{\Gamma}) \cup \check{\Gamma}$ is a compact set then $\omega(\check{\phi}) \neq \emptyset$ and, by Poincaré-Bendixson Theorem, $\omega(\check{\phi})$ either contains a singularity or it is a closed leaf. But as $\check{U}$ is a foliated set without closed leaves, we have that $\omega(\check{\phi}) \not \subset \check{U}$. Therefore $\omega(\check{\phi}) \subset \mathrm{B}_{\mathrm{c}}(\check{U})$ and the case (1.) holds for the leaf $\check{\phi}$. As $\check{\Gamma}$ is an $\check{\mathcal{F}}$-transverse simple loop on $\mathbb{R}^{2}$, take any other leaf $\check{\phi}^{\prime} \subset \check{U}$ different from $\check{\phi}$ such that $\check{\phi}^{\prime} \cap \check{\Gamma}=\left\{z^{\prime}\right\}$.

So, there exist $t, t^{\prime} \in \mathbb{R}$ such that $t<t^{\prime}<t+1$ and $\check{\gamma}(t)=z$ and $\check{\gamma}\left(t^{\prime}\right)=z^{\prime}$, where $\check{\gamma}: \mathbb{R} \rightarrow \mathbb{R}^{2}$ is the natural lift of $\check{\Gamma}$. By transversality of the path we note that for every $t \leq s \leq t^{\prime}$, if $\phi_{\check{\gamma}(s)}^{+}$is contained in $\mathrm{B}_{\mathrm{c}}(\check{\Gamma})$, then the same holds for every $s^{\prime}$ sufficiently close to $s$. Since $\left[t, t^{\prime}\right]$ is a compact interval, we get that $\check{\phi}_{z^{\prime}}^{\prime+} \subset \mathrm{B}_{\mathrm{c}}(\check{\Gamma})$. Therefore $\omega\left(\check{\phi}^{\prime}\right) \subset \mathrm{B}_{\mathrm{c}}(\check{U})$. And we conclude that the case (1.) holds for all leaves contained in $\check{U}$.

Similarly, if item (b) holds, we can show that the case (2.) holds for the leaf $\check{\phi}$, that is $\alpha(\check{\phi}) \neq \emptyset, \alpha(\check{\phi}) \subset \mathrm{B}_{\mathrm{c}}(\check{U})$ and $\omega(\check{\phi}) \not \subset \mathrm{B}_{\mathrm{c}}(\check{U})$. And, as we did above, we can conclude that the case (2.) holds for all leaves containde in $\check{U}$.

Proposition 5.3. Let $\Gamma: \mathbb{T}^{1} \rightarrow \mathbb{T}^{2}$ be an $\mathcal{F}$-transverse loop homotopic to zero on $\mathbb{T}^{2}$ and $\gamma: \mathbb{R} \rightarrow \mathbb{R}^{2}$ its natural lift. Suppose that $\Gamma$ has no $\mathcal{F}$-transverse self-intersection. If $\check{\Gamma}: \mathbb{T}^{1} \rightarrow \mathbb{R}^{2}$ is a lift of the loop $\Gamma$ to $\mathbb{R}^{2}$ then it is an $\check{\mathcal{F}}$-transverse loop on $\mathbb{R}^{2}$ and

1. There exists $\check{\Gamma}^{\prime}$ an $\mathcal{F}$-transverse simple loop such that the natural lift $\check{\gamma}: \mathbb{R} \rightarrow \mathbb{R}^{2}$ is equivalent to the natural lift $\check{\gamma}^{\prime}$ of $\check{\Gamma}^{\prime}$;

2. $\check{U}=\bigcup_{t \in \mathbb{R}} \phi_{\check{\gamma}(t)}$ is a topological open annulus in $\mathbb{R}^{2}$;

3. $\check{U} \cap \check{U}+\left(p_{1}, p_{2}\right)=\emptyset$ for every $\left(p_{1}, p_{2}\right) \in \mathbb{Z}^{2} \backslash\{(0,0)\}$.

Proof. Let $\Gamma: \mathbb{T}^{1} \rightarrow \mathbb{T}^{2}, \gamma: \mathbb{R} \rightarrow \mathbb{T}^{2}$, and $\check{\Gamma}: \mathbb{T}^{1} \rightarrow \mathbb{R}^{2}$ be the paths given in the statement. As $\Gamma$ is an $\mathcal{F}$-transverse loop homotopic to zero on $\mathbb{T}^{2}$ then, lifting such homotopy, we get that the lift $\check{\Gamma}: \mathbb{T}^{1} \rightarrow \mathbb{R}^{2}$ of $\Gamma$ to $\mathbb{R}^{2}$ is an $\check{\mathcal{F}}$-transverse loop.

By assumption, we have that $\Gamma$ has no $\mathcal{F}$-transverse self-intersection then its lift $\check{\Gamma}$ also has no $\check{\mathcal{F}}$-transverse self-intersection. Therefore the properties (1.) and (2.) follows from the Proposition 4.8 .

(3.) Suppose, by contradiction, that $\check{U} \cap \check{U}+\left(p_{1}, p_{2}\right) \neq \emptyset$ for some $\left(p_{1}, p_{2}\right) \in \mathbb{Z}^{2} \backslash(0,0)$. And let $\check{\Gamma} \subset \check{U}$ and $\left.\check{\Gamma}+\left(p_{1}, p_{2}\right) \subset \check{U}+\left(p_{1}, p_{2}\right)\right)$ be loops that lift $\Gamma$ to $\mathbb{R}^{2}$. 
By assumption, the loop $\Gamma$ has no $\mathcal{F}$-transverse self-intersection. So, in addition to any of the loops $\check{\Gamma}$ and $\check{\Gamma}+\left(p_{1}, p_{2}\right)$ not having an $\check{\mathcal{F}}$-transverse self-intersection, we also have that $\check{\Gamma}$ and $\check{\Gamma}+\left(p_{1}, p_{2}\right)$ have no $\check{\mathcal{F}}$-transverse intersection. Because otherwise there would be $s<t$ in $\mathbb{R}$ such that $\check{\gamma}$ and $\check{\gamma}+\left(p_{1}, p_{2}\right)$ have an $\check{\mathcal{F}}$-transverse intersection in $\check{\gamma}(s)=\check{\gamma}(t)+\left(p_{1}, p_{2}\right)$ which would imply that

$$
\gamma(s)=\check{\pi}(\check{\gamma}(s))=\check{\pi}\left(\check{\gamma}(t)+\left(p_{1}, p_{2}\right)\right)=\check{\pi}(\check{\gamma}(t))=\gamma(t)
$$

and $\gamma$ would have an $\mathcal{F}$-transverse self-intersection in $\gamma(s)=\gamma(t)$, which is a contradiction with the assumption.

Thus, with the Remark 4.7 and Proposition 4.6 , we have that there exist $\breve{\mathcal{F}}$-transverse simple loops $\check{\Gamma}^{\prime}$ and $\check{\Gamma}^{\prime \prime}$ that are disjoint and $\check{\mathcal{F}}$-equivalent to $\check{\Gamma}$ and $\check{\Gamma}+\left(p_{1}, p_{2}\right)$, respectively. Which means that $\check{\Gamma}^{\prime} \subset \check{U}$ and $\check{\Gamma}^{\prime \prime} \subset \check{U}+\left(p_{1}, p_{2}\right)$ and moreover

$$
\mathrm{B}_{\mathrm{c}}(\check{U}) \subset \mathrm{B}_{\mathrm{c}}\left(\check{\Gamma}^{\prime}\right) \text { and } \mathrm{B}_{\mathrm{c}}\left(\check{U}+\left(p_{1}, p_{2}\right)\right) \subset \mathrm{B}_{\mathrm{c}}\left(\check{\Gamma}^{\prime \prime}\right)
$$

where $\mathrm{B}_{\mathrm{c}}\left(\check{U}+\left(p_{1}, p_{2}\right)\right)=\mathrm{B}_{\mathrm{c}}(\check{U})+\left(p_{1}, p_{2}\right)$.

As $\check{U} \cap \check{U}+\left(p_{1}, p_{2}\right) \neq \emptyset$ and $\check{U}$ is a foliated set, there exists a leaf $\check{\phi}_{0} \subset \check{\mathcal{F}}$ satisfying $\check{\phi}_{0} \subset \check{U} \cap \check{U}+\left(p_{1}, p_{2}\right)$ and, therefore,

$$
\check{\phi}_{0} \subset \check{U} \cap \check{U}+\left(p_{1}, p_{2}\right) \Rightarrow \check{\phi}_{0}-\left(p_{1}, p_{2}\right) \subset \check{U}
$$

By Lemma 5.2 we know that for all $\check{\phi} \in \check{U}$ either $\omega(\check{\phi}) \neq \emptyset, \omega(\check{\phi}) \subset \mathrm{B}_{\mathrm{c}}(\check{U})$ and $\alpha(\check{\phi}) \not \subset \mathrm{B}_{\mathrm{c}}(\check{U})$ or $\alpha(\check{\phi}) \neq \emptyset, \alpha(\check{\phi}) \subset \mathrm{B}_{\mathrm{c}}(\check{U})$ and $\omega(\check{\phi}) \not \subset \mathrm{B}_{\mathrm{c}}(\check{U})$.

Let us suppose that the first case holds. The other one is analogous.

So, we have that $\omega\left(\check{\phi}_{0}\right) \subset \mathrm{B}_{\mathrm{c}}(\check{U})$ and $\omega\left(\check{\phi}_{0}-\left(p_{1}, p_{2}\right)\right) \subset \mathrm{B}_{\mathrm{c}}(\check{U})$ and this implies that $\omega\left(\check{\phi}_{0}\right) \subset \mathrm{B}_{\mathrm{c}}(\check{U})+\left(p_{1}, p_{2}\right)=\mathrm{B}_{\mathrm{c}}\left(\check{U}+\left(p_{1}, p_{2}\right)\right)$.

Therefore $\omega\left(\check{\phi}_{0}\right) \subset \mathrm{B}_{\mathrm{c}}\left(\check{\Gamma}^{\prime}\right)$ and $\omega\left(\check{\phi}_{0}\right) \subset \mathrm{B}_{\mathrm{c}}\left(\check{\Gamma}^{\prime \prime}\right)$. From this and the assumption that $\check{\Gamma}^{\prime}$ and $\check{\Gamma}^{\prime \prime}$ are disjoint follows that

$$
\check{\Gamma}^{\prime} \subset \mathrm{B}_{\mathrm{c}}\left(\check{\Gamma}^{\prime \prime}\right) \text { or } \check{\Gamma}^{\prime \prime} \subset \mathrm{B}_{\mathrm{c}}\left(\check{\Gamma}^{\prime}\right)
$$

If $\check{\Gamma}^{\prime} \subset \mathrm{B}_{\mathrm{c}}\left(\check{\Gamma}^{\prime \prime}\right)$, see Figure 5.1 , then $\mathrm{B}_{\mathrm{c}}(\check{U}) \subset \mathrm{B}_{\mathrm{c}}\left(\check{\Gamma}^{\prime \prime}\right)$ which implies $\mathrm{B}_{\mathrm{c}}(\check{U}) \subset \mathrm{B}_{\mathrm{c}}(\check{U}+$ $\left.\left(p_{1}, p_{2}\right)\right)$. Let us explain why.

The set $\mathrm{B}_{\mathrm{c}}(\check{U})$ is a saturated set, which means that it is a union of leaves and singularities. Thus, if $\check{\phi}^{\prime} \in \mathrm{B}_{\mathrm{c}}(\check{U})$ then $\check{\phi}^{\prime} \in \mathrm{B}_{\mathrm{c}}\left(\Gamma^{\prime \prime}\right)$ and $\check{\phi}^{\prime} \cap \check{\Gamma}^{\prime \prime}=\emptyset$, which imply that $\check{\phi}^{\prime}$ does not belong to $\check{U}+\left(p_{1}, p_{2}\right)$, it belongs to $\mathrm{B}_{\mathrm{c}}\left(\check{U}+\left(p_{1}, p_{2}\right)\right)$. And, if $w \in \operatorname{sing}(\check{\mathcal{F}}) \cap \mathrm{B}_{\mathrm{c}}(\check{U})$ then $w \in \mathrm{B}_{\mathrm{c}}\left(\check{U}+\left(p_{1}, p_{2}\right)\right)$, because $w \in \mathrm{B}_{\mathrm{c}}\left(\check{\Gamma}^{\prime \prime}\right)$ and $w \notin \check{U}+\left(p_{1}, p_{2}\right)$ since the set $\check{U}+\left(p_{1}, p_{2}\right)$ is a foliated set.

Then we have that $\mathrm{B}_{\mathrm{c}}(\check{U}) \subset \mathrm{B}_{\mathrm{c}}\left(\check{U}+\left(p_{1}, p_{2}\right)\right)=\mathrm{B}_{\mathrm{c}}(\check{U})+\left(p_{1}, p_{2}\right)$ and this is a contradiction, because $\left(p_{1}, p_{2}\right)$ is a non-zero vector on $\mathbb{Z}^{2}$ and $\mathrm{B}_{\mathrm{c}}(\check{U})$ is a non-empty compact 


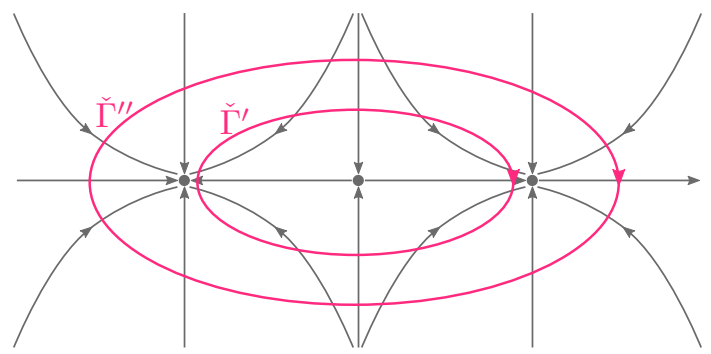

Figure 5.1: $\check{\Gamma}^{\prime} \subset \mathrm{B}_{\mathrm{c}}\left(\check{\Gamma}^{\prime \prime}\right)$

subset of $\mathbb{R}^{2}$.

And, if $\check{\Gamma}^{\prime \prime} \subset \mathrm{B}_{\mathrm{c}}\left(\check{\Gamma}^{\prime}\right)$ then, similarly, we can show that $\mathrm{B}_{\mathrm{c}}(\check{U})+\left(p_{1}, p_{2}\right) \subset \mathrm{B}_{\mathrm{c}}(\check{U})$, which is a contradiction again.

Therefore, we conclude that we must have $\check{U} \cap \check{U}+\left(p_{1}, p_{2}\right)=\emptyset$.

The next proposition relates homeomorphisms of $\mathbb{T}^{2}$ isotopic to identity whose the rotation set is a non-degenerate line segment with the existence of topological horseshoes.

Proposition 5.4. Let $f \in \operatorname{Homeo}\left(\mathbb{T}^{2}\right)$ be topologically transitive with a non-empty fixed point set. If there is an integer $k \geq 1$ such that the power $g=f^{k}$ of $f$ is isotopic to identity and the rotation set of a lift $\check{g} \in \operatorname{Homeo}\left(\mathbb{R}^{2}\right)$ of $g$ is a non-degenerate line segment with a rational slope then $f$ has a topological horseshoe.

Before proving this proposition, we will prove some useful facts.

Lemma 5.5. Let $h \in \operatorname{Homeo}\left(\mathbb{T}^{2}\right)$. Suppose that $h$ admits a lift $\hat{h} \in \operatorname{Homeo}(\mathbb{A})$ to the annulus. Moreover, suppose that

1. The map $\hat{h}$ commutes with the integer vertical translations, that is,

$$
\hat{h}(\hat{w}+(0, p))=\hat{h}(\hat{w})+(0, p), \forall p \in \mathbb{Z} \text { and } \hat{w} \in \mathbb{A} ;
$$

2. There is a real number $M>0$ such that for all $\hat{w} \in \mathbb{A}$ we have

$$
\left|\mathrm{p}_{2}\left(\hat{h}^{n}(\hat{w})-\hat{w}\right)\right| \leq M, \forall n \in \mathbb{Z}
$$

Then, if $w, w^{\prime} \in \mathbb{T}^{2}$ are such that $w \in \omega_{h}\left(w^{\prime}\right)$, then for every $\hat{w} \in \hat{\pi}^{-1}(w)$ there exists some $\hat{w}^{\prime} \in \hat{\pi}^{-1}\left(w^{\prime}\right)$ such that $\hat{w} \in \omega_{\hat{h}}\left(\hat{w}^{\prime}\right)$.

Proof. Let $w, w^{\prime} \in \mathbb{T}^{2}$ be as in the hypothesis. Choose some $\hat{w} \in \hat{\pi}^{-1}(w)$, then there is a fundamental domain $\hat{D}=\mathbb{T}^{1} \times[d, d+1)$ in $\mathbb{A}$ that contains $\hat{w}$. So

$$
d \leq \mathrm{p}_{2}(\hat{w})<d+1
$$

As $w \in \omega_{h}\left(w^{\prime}\right)$ then there exist an increasing sequence $\left(m_{k}\right)_{k \in \mathbb{N}}$ of positive integers such that $m_{k} \rightarrow+\infty$ and

$$
\lim _{k \rightarrow+\infty} h^{m_{k}}\left(w^{\prime}\right)=w
$$


And we may assume, possibly by taking a subsequence, that

$$
d_{\mathbb{T}^{2}}\left(h^{m_{k}}\left(w^{\prime}\right), w\right)<1 / k, \text { for all } k \in \mathbb{N}
$$

By assumption, we have that $\hat{h}$ commutes with the integer vertical translations. This assumption and the above property imply that there exist $\hat{w}^{\prime} \in \hat{\pi}^{-1}\left(w^{\prime}\right)$ and a sequence of integers $\left(p_{k}\right)_{k \in \mathbb{N}} \subset \mathbb{Z}$ such that

$$
d_{\mathbb{A}}\left(\hat{h}^{m_{k}}\left(\hat{w}^{\prime}\right)+\left(0, p_{k}\right), \hat{w}\right)<1 / k, \forall k>k_{0} .
$$

So, (5.2) and 5.3 imply that

$$
d-\frac{1}{k}<\mathrm{p}_{2}\left(\hat{h}^{m_{k}}\left(\hat{w}^{\prime}\right)\right)+p_{k}<d+1+\frac{1}{k}, \forall k>k_{0} .
$$

Note that we can assume that $\hat{w}^{\prime} \in \hat{\pi}^{-1}\left(w^{\prime}\right) \cap \hat{D}$, because if not then there would be an integer $p$ such that the integer translation $\hat{w}_{p}^{\prime}=\hat{w}^{\prime}+(0, p)$ of $\hat{w}^{\prime}$ would be in $\hat{D}$ and therefore, there would be a sequence of integers $\left(p_{k}^{\prime}\right)_{k \in \mathbb{N}} \subset \mathbb{Z}$, namely $p_{k}^{\prime}=p_{k}-p$ for each $k \in \mathbb{N}$, which would imply that

$$
d_{\mathbb{A}}\left(\hat{h}^{n_{k}}\left(\hat{w}_{p}^{\prime}+\left(0, p_{k}^{\prime}\right)\right), \hat{w}^{\prime}\right)<1 / k, \quad \forall k>k_{0} .
$$

So, from now on, we will assume without loss of generality that $\hat{w}^{\prime} \in \hat{\pi}^{-1} \cap \hat{D}$. Then

$$
d \leq \mathrm{p}_{2}\left(\hat{w}^{\prime}\right) \leq d+1
$$

By the assumption (5.1) and the inequality 5.5 we have that

$$
d-M<\mathrm{p}_{2}\left(\hat{h}^{m_{k}}\left(\hat{w}^{\prime}\right)\right)<d+1+M \text {. }
$$

So, (5.4) and (5.6) imply that, for all $k$

$$
-d-1-M+d-\frac{1}{k}<p_{k}<-d+M+d+1+\frac{1}{k} \Rightarrow\left|p_{k}\right|<M+1+\frac{1}{k}<M+2
$$

because we have that $k>k_{0}>1$ and then $1 / k<1$.

This means that the discrete set $\left\{p_{k} \mid k>k_{0}\right\} \subset \mathbb{Z}$ is finite. Indeed the cardinality of $\left\{p_{k} \mid k>k_{0}\right\}$, namely $\#\left\{p_{k} \mid k>k_{0}\right\}$ is less than or equal to $2\lfloor M+2\rfloor+1$, where $\lfloor x\rfloor$ is the largest integer less than or equal to the positive real number $x$.

Then, by the Pigeonhole Principle, there is a subsequence $\left(p_{k_{i}}\right)_{i \in \mathbb{N}}$ of $\left(p_{k}\right)_{k \in \mathbb{N}}$ such that $p_{k_{i}}=p_{0} \in \mathbb{Z}$, for all $i \in \mathbb{N}$.

So, what we have is that

$$
d_{\mathbb{A}}\left(\hat{h}^{m_{k_{i}}}\left(\hat{w}^{\prime}+\left(0, p_{0}\right)\right), \hat{w}\right)<1 / k_{i}, \forall i \in \mathbb{N}
$$


and this imply that $\hat{w}$ is in the $\omega$-limit of the lift $\hat{w}^{\prime}+\left(0, p_{0}\right)$ of $w^{\prime}$ by the map $\hat{h}$, namely $\hat{w} \in \omega_{h}\left(\hat{w}^{\prime}+\left(0, p_{0}\right)\right)$.

As a Corollary of Lemma 2.1 and Lemma 5.5 we have:

Corollary 5.6. Let $f \in \operatorname{Homeo}\left(\mathbb{T}^{2}\right)$ be topologically transitive. If there is a power $h=f^{k}$, $k \geq 1$, of $f$ that admits a lift $\hat{h} \in \operatorname{Homeo}(\mathbb{A})$ satisfying the assumptions (1.) and (2.) of Lemma 5.5 then $\hat{h}$ is non-wandering.

Proof. Let $\hat{w} \in \mathbb{A}$ be any point. Then, by Lemma 2.1. there exist some $r \in\{0, \cdots, k-1\}$ such that

$$
\hat{\pi}(\hat{w}) \in \omega_{h}\left(f^{r}(z)\right)
$$

By Lemma 5.5 we know that there is some lift $\hat{z}_{r} \in \hat{\pi}^{-1}\left(f^{r}(z)\right)$ such that $\hat{w} \in \omega_{\hat{h}}\left(\hat{z}_{r}\right)$. Therefore $\hat{w} \in \mathbb{A}$ is non-wandering for $\hat{h}$.

As $\hat{w} \in \mathbb{A}$ is any point, we conclude that $\hat{h}$ is non-wandering.

Remark 5.7. Note that if $\hat{z}_{r} \in \mathbb{A}$ is a point satisfying $\hat{\pi}\left(\hat{z}_{r}\right)=f^{r}(z)$, where $r \in\{0, \cdots, k-$ $1\}$, the set of all lifts to $\mathbb{A}$ of the set $\left\{z, f(z), \cdots, f^{k-1}(z)\right\}$, namely

$$
\hat{G}=\bigcup_{p \in \mathbb{Z}}\left(\bigcup_{r=0}^{k-1}\left\{\hat{z}_{r}+(0, p)\right\}\right)
$$

is an enumerable set.

Proof of Proposition 5.4. Suppose, by contradiction, that $f$ does not have a topological horseshoe. Then no power of $f$ has a topological horseshoe.

Let $k \geq 1$ be an integer such that the power $g=f^{k}$ of $f$ is isotopic to identity and the rotation set of a lift $\check{g} \in \operatorname{Homeo}\left(\mathbb{R}^{2}\right)$ of $g$, is a non-degenerate line segment with a rational slope.

First of all, we will prove that $g=f^{k}$ is topologically conjugate to a homeomorphism $h \in \operatorname{Homeo}\left(\mathbb{T}^{2}\right)$ that has a lift $\check{h} \in \operatorname{Homeo}\left(\mathbb{R}^{2}\right)$ such that $\rho(\check{h})$ is a horizontal segment containing the origin and such that $(0,0) \in \rho(\breve{h})$ is realized by periodic orbit.

Let $z_{0} \in \operatorname{fix}(f)$, then $z_{0} \in \operatorname{fix}(g)$, and as $g$ is isotopic to identity, there is some $\left(p_{1}, p_{2}\right) \in \mathbb{Z}^{2}$ such that $\check{g}\left(\check{z}_{0}\right)=\check{z}_{0}+\left(p_{1}, p_{2}\right)$, where $\check{z}_{0} \in \check{\pi}^{-1}\left(z_{0}\right)$ is independent of the choice. There is no loss of generality in supposing that $\left(p_{1}, p_{2}\right)=(0,0)$, because if it is not, then the lift $\check{g}^{\prime}=\check{g}-\left(p_{1}, p_{2}\right)$ is such that $\check{g}^{\prime}\left(\check{z}_{0}\right)=\check{z}_{0}$ and as $\rho\left(\check{g}^{\prime}\right)=\rho(\check{g})-\left(p_{1}, p_{2}\right)$ then, we could take the lift $\check{g}^{\prime}$ of $g$ instead of $\check{g}$. Thus $(0,0) \in \rho(\check{g})$ is realized by $z_{0} \in \mathbb{T}^{2}$.

As, by assumption $\rho(\check{g})$ is a non-degenerate line segment with a rational slope then, by Corollary 3.4 , there is $A \in \mathrm{GL}(2, \mathbb{Z})$ such that $\rho\left(A \check{g} A^{-1}\right)=A \rho(\check{g})$ is a horizontal segment and if $f_{A}$ is the homeomorphism of $\mathbb{T}^{2}$ induced by $A$ then we have that $f_{A}\left(z_{0}\right) \in \mathbb{T}^{2}$ is fixed by $f_{A} g f_{A}^{-1}$ and moreover 


$$
\begin{aligned}
\rho\left(f_{A}\left(z_{0}\right), A \check{g} A^{-1}\right) & =\lim _{n \rightarrow+\infty} \frac{\left(A \check{g} A^{-1}\right)^{n}\left(A \check{z}_{0}\right)-A \check{z}_{0}}{n} \\
& =A\left(\lim _{n \rightarrow+\infty} \frac{\check{g}^{n}\left(\check{z}_{0}\right)-\check{z}_{0}}{n}\right) \\
& =A\left(\begin{array}{l}
0 \\
0
\end{array}\right) \\
& =\left(\begin{array}{l}
0 \\
0
\end{array}\right),
\end{aligned}
$$

where $A \check{z}_{0} \in \check{\pi}^{-1}\left(f_{A}\left(z_{0}\right)\right)$ is independent of the choice.

Thus, under a topological conjugacy, we can assume that $g=f^{k}$ is a homeomorphism of $\mathbb{T}^{2}$ isotopic to identity such the rotation set of some lift $\check{g} \in \operatorname{Homeo}\left(\mathbb{R}^{2}\right)$ of $g$ is a horizontal segment containing $(0,0)$ and such that $(0,0) \in \rho(\check{g})$ is realized by $z_{0} \in \operatorname{fix}(g)$.

By Corollary 3.7, $\check{g}$ has uniformly bounded deviations in the perpendicular direction of $\rho(\check{g})$, so there is a real number $M>0$ such that

$$
\left|\mathrm{p}_{2}\left(\check{g}^{n}(\check{w})-\check{w}\right)\right| \leq M, \quad \forall n \in \mathbb{Z} \text { and } \forall \check{w} \in \mathbb{R}^{2}
$$

Furthermore, as $\rho(\check{g})$ is a non-degenerate line segment containing the origin, let $a \in$ $\rho(\check{g}) \backslash\{(0,0)\}$ be an extremal point. Then we know (item (3.) of Proposition 3.1) there is some point $z_{a} \in \mathbb{T}^{2} \backslash\left\{z_{0}\right\}$ such that $\rho\left(z_{a}, \check{g}\right)=a \neq(0,0)$.

By assumption, we have that $f \in \operatorname{Homeo}\left(\mathbb{T}^{2}\right)$ is topologically transitive. So, there is a point $z \in \mathbb{T}^{2}$ such that $\omega_{f}(z)=\mathbb{T}^{2}$. Then, by Lemma 2.1, we have

$$
\bigcup_{r=0}^{k-1} \omega_{g}\left(f^{r}(z)\right)=\mathbb{T}^{2} .
$$

Now, let $I_{0} \in \mathcal{I}_{g}$ be an identity isotopy of $g$ such that $z_{0} \in \operatorname{fix}\left(I_{0}\right)$, then $I_{0}$ is lifted to an identity isotopy $\check{I}_{0}$ of $\check{g}$. Let $I$ be a maximal isotopy of $g$ such that $I_{0} \preceq I$ and $\check{I}$ its lift to $\mathbb{R}^{2}$. We consider a foliation $\mathcal{F}$ tranverse to $I$ and its lift $\check{\mathcal{F}}$ to $\mathbb{R}^{2}$.

The quotient space of $\mathbb{R}^{2}$ by a unit horizontal translation is homeomorphic to the annulus $\mathbb{A}$. We get an identity isotopy $\hat{I}=\left(\hat{g}_{t}\right)_{t \in[0,1]}$ on $\mathbb{A}$ by projection, as well a homeomorphism $\hat{g}=\hat{g}_{1}$ and a transverse foliation $\hat{\mathcal{F}}$. Therefore $\hat{g}$ is isotopic to identity.

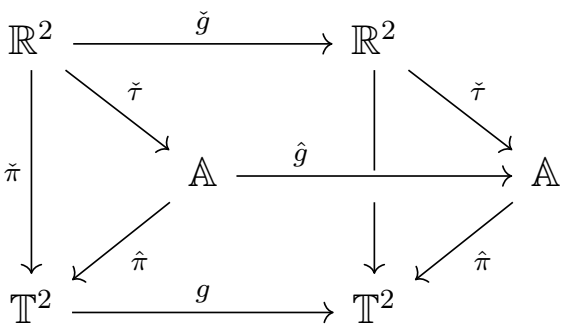

And as the diagram in 5.9 commutes we have that $\hat{g}(\hat{w}+(0, p))=\hat{g}(\hat{w})+(0, p)$, for 
all $p \in \mathbb{Z}$.

Moreover, it follows from the equation (5.7) that

$$
\left|\mathrm{p}_{2}\left(\hat{g}^{n}(\hat{w})-\hat{w}\right)\right| \leq M, \quad \forall n \in \mathbb{Z} \text { and } \forall \check{\tau}(\check{w})=\hat{w} \in \mathbb{A}
$$

This means that for all points $\hat{w} \in \mathbb{A}$, the orbit of $\hat{w}$ is contained in a compact subset of $\mathbb{A}$. And, consequently, we have that the $\omega_{g}(\hat{w})$ is not empty, for all $\hat{w} \in \mathbb{A}$. Furthermore, by Corollary 5.6, we have that $\hat{g} \in \operatorname{Homeo}(\mathbb{A})$ is non-wandering.

By assumption $g \in \operatorname{Homeo}\left(\mathbb{T}^{2}\right)$ has no topological horseshoe. Thus, by Remark 2.6 we have that $\hat{g}$ can not have a topological horseshoe. So, $\hat{g}: \mathbb{A} \rightarrow \mathbb{A}$ is a non-wandering homeomorphism isotopic to identity such that the $\omega$-limit of any point $\hat{w} \in \mathbb{A}$ is non-empty and has no topological horseshoe. Then Theorem 3.9 implies that the $\operatorname{map} \operatorname{rot}_{\check{g}}: \mathbb{A} \rightarrow \mathbb{R}$ that associates to each point $\hat{w} \in \mathbb{A}$ its rotation number $\operatorname{rot}\left(\hat{w}^{\prime}, \check{g}\right)$ is well defined and continuous. In the following we will prove that it must be constant. Let

$$
\hat{G}=\bigcup_{p \in \mathbb{Z}}\left(\bigcup_{r=0}^{k-1}\left\{\hat{z}_{r}+(0, p)\right\}\right),
$$

be the set of all lifts of the set $\left\{z, f(z), \cdots, f^{k-1}(z)\right\}$, where $\hat{z}_{r} \in \hat{\pi}^{-1}\left(f^{r}(z)\right)$ for $r \in$ $\{0,1, \cdots, k-1\}$.

Given any $\hat{w} \in \mathbb{A}$, Lemma 5.5 implies that there exists some point $\hat{w}^{\prime} \in \hat{G}$ such that $\hat{w} \in \omega_{\hat{g}}\left(\hat{w}^{\prime}\right)$. And by item (2) of Theorem 3.9 the rotation number of $\hat{w}$ must be the same as the rotation number of $\hat{w}^{\prime}$. But, as observed in Remark 5.7, the set $\hat{G}$ is an enumerable set. Therefore, as the map $\operatorname{rot}_{\check{g}}$ is continuous, it must be constant.

But this is impossible, because if we take $\hat{z}_{0} \in \hat{\pi}^{-1}\left(z_{0}\right)$ and $\hat{z}_{a} \in \hat{\pi}^{-1}\left(z_{a}\right)$ then $\hat{z}_{0}, \hat{z}_{a} \in$ $\mathrm{ne}^{+}(\hat{g})$, as observed. Moreover, $\operatorname{rot}\left(\hat{z}_{0}, \check{g}\right)=\mathrm{p}_{1}\left(\rho\left(z_{0}, \check{g}\right)\right)=0$ and $\operatorname{rot}\left(\hat{z}_{a}, \check{g}\right)=\mathrm{p}_{1}\left(\rho\left(z_{a}, \check{g}\right)\right)=$ $\mathrm{p}_{1}(a) \neq 0$ because $a \in \rho(\check{g})$ is a non-zero vector of a horizontal non-degenerate line segment of $\mathbb{R}$ containing the origin.

Therefore, we have proved by contradiction that $f$ must have a topological horseshoe.

\subsection{Proof of Theorem A}

Let us restate Theorem A:

Theorem A. Let $f \in \mathrm{Homeo}_{0}\left(\mathbb{T}^{2}\right)$ be topologically transitive. If $f$ has a fixed point and a periodic point non-fixed then $f$ has a topological horseshoe.

Proof. Let $z_{0} \in \mathbb{T}^{2}$ be a fixed point, that is $z_{0} \in \operatorname{fix}(f) ; q_{1}>1$ be the minimal period of a non-fixed periodic point $z_{1} \in \mathbb{T}^{2}$, that is $z_{1} \in \operatorname{fix}\left(f^{q_{1}}\right) \backslash$ fix $(f)$, and $z \in \mathbb{T}^{2}$ be a point such that $\omega_{f}(z)=\alpha_{f}(z)=\mathbb{T}^{2}$. 
Let $\mathcal{I}_{f}$ be the set of identity isotopies for $f$, pick $I_{0} \in \mathcal{I}_{f}$ such that $z_{0} \in \operatorname{fix}\left(I_{0}\right)$ and let $I \in \mathcal{I}_{f}$ be a maximal isotopy for $f$ such that $I_{0} \preceq I$ and let $\mathcal{F}$ be a transverse foliation to $I$.

We will suppose that any admissible path have no $\mathcal{F}$-transverse self-intersection, because if some admissible path has an $\mathcal{F}$-transverse self-intersection then Theorem 4.28 implies that $f$ possesses a topological horseshoe and we finish the proof.

Let $\check{I}=\left(\check{f}_{t}\right)_{t \in[0,1]}$ be an identity isotopy that lifts $I$ to $\mathbb{R}^{2}, \check{f}:=\check{f}_{1}$ be the lift of $f$ to $\mathbb{R}^{2}$ given by the isotopy $I$ and $\check{\mathcal{F}}$ be the foliation of $\mathbb{R}^{2}$ that lifts $\mathcal{F}$. Observe that $\check{\mathcal{F}}$ is transverse to $\check{I}$ and as fix $(I) \neq \emptyset$ we have that $\operatorname{fix}(\check{I})=\check{\pi}^{-1}(\operatorname{fix}(I))$ is non-empty and then $\check{\mathcal{F}}$ is a singular oriented foliation of $\mathbb{R}^{2}$. Furthermore any lift of any admissible path has no $\check{\mathcal{F}}$-transverse self-intersection.

As $z_{1} \in \operatorname{fix}\left(f^{q_{1}}\right)$ with $q_{1}>1$ we know that the trajectory of $z_{1}$ by the isotopy $I$ until order $q_{1}, I^{q_{1}}\left(z_{1}\right)$, is a loop on $\mathbb{T}^{2}$. Then we have two possibilities for it:

a) $I^{q_{1}}\left(z_{1}\right)$ is homotopic to zero on $\mathbb{T}^{2}$;

b) Or not, which means that $I^{q_{1}}\left(z_{1}\right)$ is an essential loop on $\mathbb{T}^{2}$.

\subsubsection{Loop homotopic to zero on $\mathbb{T}^{2}$}

Suppose that $I^{q_{1}}\left(z_{1}\right)$ is homotopic to zero on $\mathbb{T}^{2}$. Let $\Gamma_{1}: \mathbb{T}^{1} \rightarrow \operatorname{dom}(I)$ be an $\mathcal{F}$ transverse loop that is homotopic to $I^{q_{1}}\left(z_{1}\right)$ such that $\Gamma_{1}(0)=z_{1}$ and let $\gamma_{1}: \mathbb{R} \rightarrow \operatorname{dom}(I)$ be its natural lift. Thus we have the path $\gamma_{1}(0)=z_{1}$ and therefore it is a whole transverse trajectory of the point $z_{1}$, which means $\gamma_{1}:=I_{\mathcal{F}}^{\mathbb{Z}}\left(z_{1}\right)$.

As $\Gamma_{1}$ is homotopic to $I^{q_{1}}\left(z_{1}\right)$ on $\operatorname{dom}(I)$, then it is an $\mathcal{F}$-transverse loop that it is itself homotopic to zero on $\mathbb{T}^{2}$. Moreover it has no $\mathcal{F}$-transverse self-intersection, by assumption. So, follows from Proposition 5.3 that any lift $\check{\Gamma}_{1}$ of $\Gamma_{1}$ to $\mathbb{R}^{2}$ is $\check{\mathcal{F}}$-equivalent to a multiple of an $\check{\mathcal{F}}$-transverse simple loop and if $\check{\gamma}_{1}: \mathbb{R} \rightarrow \mathbb{R}^{2}$ is the natural lift of the loop $\check{\Gamma}_{1}, \check{U}_{1}=\bigcup_{t \in \mathbb{R}} \phi_{\check{\gamma}_{1}(t)}$ is a topological open annulus on $\mathbb{R}^{2}$ which is disjoint from all integers translates of it.

With those assumptions, we have the following claims:

Claim 5.8. Let $z \in \mathbb{T}^{2}$ be the point such that $\omega_{f}(z)=\alpha_{f}(z)=\mathbb{T}^{2}$. Given any lift $\check{z} \in \check{\pi}^{-1}(z)$ then $\check{z}$ is non-wandering for $\check{f}$.

Proof. As $z \in \omega_{f}(z) \cap \alpha_{f}(z)$, there are increasing sequences of integers $\left(m_{k}\right)_{k \in \mathbb{N}}$ and $\left(n_{l}\right)_{l \in \mathbb{N}}$ such that $m_{k} \nearrow+\infty$ and $n_{l} \nearrow+\infty$ and $f^{m_{k}}(z) \rightarrow z$ and $f^{-n_{l}}(z) \rightarrow z$. That is, we can assume, eventually by taking subsequences, that

$$
\begin{aligned}
& d_{\mathbb{T}^{2}}\left(f^{m_{k}}(z), z\right)<1 / k, \quad \forall k \in \mathbb{N} \\
& d_{\mathbb{T}^{2}}\left(f^{-n_{l}}(z), z\right)<1 / l, \quad \forall l \in \mathbb{N}
\end{aligned}
$$


So, there are two sequences of integer vectors $\left(p_{k}\right)_{k \in \mathbb{N}},\left(q_{l}\right)_{l \in \mathbb{N}} \in \mathbb{Z}^{2}$ such that

$$
\begin{aligned}
& d\left(\check{f}^{m_{k}}(\check{z}), \check{z}+p_{k}\right)<1 / k, \quad \forall k \in \mathbb{N} \\
& d\left(\check{f}^{-n_{l}}(\check{z}), \check{z}+q_{l}\right)<1 / l, \quad \forall l \in \mathbb{N}
\end{aligned}
$$

We have two possibilities:

a) Either there is a constant real number $K$ or a constant real number $L$ such that

$$
\left\|p_{k}\right\|<K \text { or }\left\|q_{l}\right\|<L
$$

And then, by the Pigeonhole Principle, either there is a subsequence $\left(p_{k_{i}}\right)_{i \in \mathbb{N}}$ of $\left(p_{k}\right)_{k \in \mathbb{N}}$ such that $p_{k_{i}}=p \in \mathbb{Z}^{2}$ for all $i \in \mathbb{N}$ or there is a subsequence $\left(q_{l_{j}}\right)_{j \in \mathbb{N}}$ of $\left(q_{l}\right)_{l \in \mathbb{N}}$ such that $q_{l_{j}}=q \in \mathbb{Z}^{2}$ for all $j \in \mathbb{N}$.

b) There are subsequences $\left(p_{k_{i}}\right)_{i \in \mathbb{N}}$ and $\left(q_{l_{j}}\right)_{j \in \mathbb{N}}$ of $\left(p_{k}\right)_{k>k_{0}}$ and $\left(q_{l}\right)_{l>l_{0}}$, respectively, such that

$$
\left\|p_{k_{i}}\right\| \rightarrow+\infty \text { and }\left\|q_{l_{j}}\right\| \rightarrow+\infty
$$

Item (a) implies that

$$
\begin{aligned}
& d\left(\check{f}^{m_{k}}(\check{z}), \check{z}+p\right)<1 / k, \quad \forall k>k_{0} \text { or } \\
& d\left(\check{f}^{-n_{l}}(\check{z}), \check{z}+q\right)<1 / l, \quad \forall l>l_{0}
\end{aligned}
$$

which implies that $\check{z}+p \in \omega_{\check{f}}(\check{z})$ or $\check{z}+q \in \alpha_{\check{f}}(\check{z})$. And therefore, $\check{z} \in \omega_{\check{f}}(\check{z}-p)$ or $\check{z} \in \alpha_{\check{f}}(\check{z}-q)$, because $\check{f}$ is a homeomorphism that commutes with the integer vector translations. So, $\check{z}$ is non-wandering for $\check{f}$.

Now we will show, by contradiction, that item (b) does not happen. Indeed, suppose by contradiction that it does happen. So, we have that

$$
\begin{aligned}
& d\left(\check{f}^{m_{k_{i}}}(\check{z}), \check{z}+p_{k_{i}}\right)<1 / k_{i}, \forall k_{i}>k_{0} \\
& d\left(\check{f}^{-n_{l_{j}}}(\check{z}), \check{z}+q_{l_{j}}\right)<1 / l_{j}, \forall l_{j}>l_{0},
\end{aligned}
$$

where $\left(p_{k_{i}}\right)_{i \in \mathbb{N}}$ and $\left(q_{l_{j}}\right)_{j \in \mathbb{N}}$ are subsequences of $\left(p_{k}\right)_{k>k_{0}}$ and $\left(q_{l}\right)_{l>l_{0}}$, respectively, such that

$$
\left\|p_{k_{i}}\right\| \rightarrow+\infty \text { and }\left\|q_{l_{j}}\right\| \rightarrow+\infty
$$

Let $\check{\gamma}:=\check{I}_{\mathcal{F}}^{\mathbb{Z}}(\check{z})$ and $\check{\phi} \subset \check{\mathcal{F}}$ such that $\check{z} \in \check{\phi}$. Thus, the properties in 5.12 and the inequalities in 5.11 together with Lemma 4.23 implies that there are real numbers $t_{i} \searrow-\infty$ and $t_{j} \nearrow+\infty$ such that

$$
\check{\gamma}\left(t_{i}\right) \in \check{\phi}+p_{k_{i}} \text { and } \check{\gamma}\left(t_{j}\right) \in \check{\phi}+q_{l_{j}} \text {. }
$$


That is, the whole $\check{\mathcal{F}}$-transverse trajectory of $\check{z} \check{\gamma}$ meets, arbitrarily in the past and in the future, integers translates of $\check{\phi}$ more and more distant of $\check{\phi}$.

Moreover, we have in $\mathbb{T}^{2}$ that $z_{1} \in \omega_{f}(z) \cap \alpha_{f}(z)$ and by Lemma 4.23 there are $a<b$ and $t$ real numbers that

$$
\left.I_{\mathcal{F}}^{\mathbb{Z}}(z)\right|_{[a, b]} \text { is } \mathcal{F} \text {-equivalent to }\left.\gamma_{1}\right|_{[t, t+1]} \text {. }
$$

So, there is a lift $\check{\gamma}_{1}$ of $\gamma_{1}$ such that

$$
\left.\check{\gamma}\right|_{[a, b]} \text { is } \check{\mathcal{F}} \text {-equivalent to }\left.\check{\gamma}_{1}\right|_{[t, t+1]} \text {, }
$$

By the discussion, above this claim, we know that $\check{\gamma}_{1}$ is the natural lift of an $\mathcal{F}$ transverse simple loop $\check{\Gamma}_{1}$ (up to equivalence) and $\check{U}_{1}=\bigcup_{t \in \mathbb{R}} \phi_{\check{\gamma}_{1}(t)}$ is a topological open annulus in $\mathbb{R}^{2}$ which is disjoint from all integers translates of it.

As $\check{U}_{1}=\bigcup_{t \in \mathbb{R}} \phi_{\check{\gamma}_{1}(t)}$ is disjoint from all integers translates of it, we have that the union $\check{U}_{1} \cup \mathrm{B}_{\mathrm{c}}\left(\check{U}_{1}\right)$ contains at most one integer translate of $\check{\phi}$, the leaf that contains the point $\check{z}$

So, there must be $p_{k}, q_{l} \in \mathbb{Z}^{2}$ integer vectors, such that $\left\|p_{k}\right\|$ and $\left\|q_{l}\right\|$ are large enough so that $\check{\phi}+p_{k}$ and $\check{\phi}+q_{l}$ are in $\mathrm{UB}_{\mathrm{c}}\left(\check{U}_{1}\right)$ and there are $t_{1}, t_{2} \in \mathbb{R}$ such that $t_{1}<a<b<t_{2}$ and

$$
\check{\gamma}\left(t_{1}\right) \in \check{\phi}+q_{l} \text { and } \check{\gamma}\left(t_{2}\right) \in \check{\phi}+p_{k} \text {. }
$$

Property 5 .13) means that the path $\check{\gamma}$ draws the loop $\check{\Gamma}_{1}$, which means that given $J_{\check{\Gamma}_{1}}^{\check{\gamma}}=\left\{t \in \mathbb{R} \mid \check{\gamma}(t) \in \check{U}_{1}\right\}$, there exists a connected component $J \subset J_{\check{\Gamma}_{1}}^{\check{\gamma}}$ that contains $[a, b]$ and such that $\left.\check{\gamma}\right|_{J}$ draws $\check{\Gamma}_{1}$. Moreover, as $t_{1}$ and $t_{2}$ are separated by $[a, b]$, they must be separated by $J$ and $J$ must be a bounded interval.

Remember that by assumption, $\check{\gamma}$ has no $\check{\mathcal{F}}$-transverse self-intersection. Thus, we have the following claim:

Claim. $\check{\gamma}$ does not cross $\check{\Gamma}_{1}$.

Proof of Claim. Suppose, by contradiction, that $\check{\gamma}$ cross $\check{\Gamma}_{1}$. So, by Proposition 4.10 the crossing component must be unique and coincides with the drawing component. Thus $J$ is the unique crossing component. Let $J=(c, d)$.

By property 5.14 we know that $\check{\gamma}\left(t_{1}\right)$ and $\check{\gamma}\left(t_{2}\right)$ are in $\mathrm{UB}_{\mathrm{c}}\left(\check{U}_{1}\right)$. Moreover, as $J=(c, d)$ is a crossing component, we have that either $\check{\gamma}(c)$ or $\check{\gamma}(d)$ is in $\mathrm{B}_{\mathrm{c}}\left(\check{U}_{1}\right)$ and the other is in $\mathrm{UB}_{\mathrm{c}}\left(\check{U}_{1}\right)$. We will assume that $\check{\gamma}(c)$ is $\mathrm{UB}_{\mathrm{c}}\left(\check{U}_{1}\right)$, the other case is similar.

As $t_{1}$ and $t_{2}$ are separated by $J$, there must exist $e, f \in \mathbb{R}$ such that $d<e<f<t_{2}$, $\left.\check{\gamma}\right|_{(e, f)} \subset \check{U}_{1}$ and

$$
\left\{\begin{array}{l}
\check{\gamma}(e) \text { and } \check{\gamma}(d) \text { are in } \mathrm{B}_{\mathrm{c}}\left(\check{U}_{1}\right) \\
\check{\gamma}(f) \text { and } \check{\gamma}\left(t_{2}\right) \text { are in } \mathrm{UB}_{\mathrm{c}}\left(\check{U}_{1}\right)
\end{array}\right.
$$

Meaning that $J^{\prime}=(e, f)$ is another crossing component of $J_{\check{\Gamma}_{1}}^{\check{\gamma}}$ which is a contradiction 
(see Figure 5.2. Therefore $\check{\gamma}$ does not cross $\check{\Gamma}_{1}$.

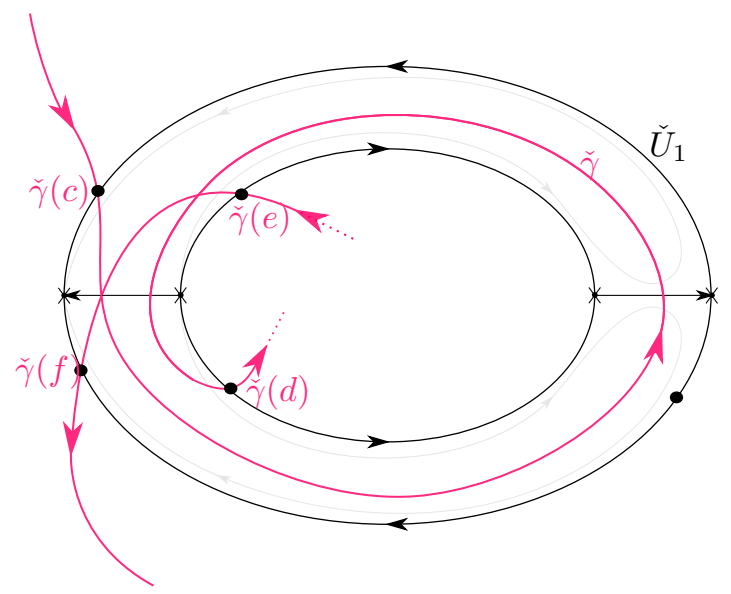

Figure 5.2: $J$ and $J^{\prime}$ are crossing components of $J_{\check{\Gamma}_{1}}^{\check{\gamma}}$.

End of the proof of Lemma 5.8. As $\check{\gamma}: \mathbb{R} \rightarrow \mathbb{R}^{2}$ has no $\check{\mathcal{F}}$-transverse self-intersection and does not cross $\check{\Gamma}_{1}$ thus, by item (3) of Proposition 4.10, we have that the drawing component $J$ coincides with $\mathbb{R}$ in a neighborhood of at least one endpoint of $\mathbb{R}$, but the existence of $t_{1}$ and $t_{2}$ prohibits this to happen. Therefore we get a contradiction and we conclude that item (b) does not happen.

As $\check{z} \in \check{\pi}^{-1}(z)$ was taken arbitrarily, we conclude that any lift $\check{z}$ of the transitive point $z$ is non-wandering.

Let us fix a lift $\check{\Gamma}_{1}$ of $\Gamma_{1}$ to $\mathbb{R}^{2}$ and let $\check{U}_{1}$ be the open annulus that contains $\check{\Gamma}_{1}$.

Claim 5.9. Let $U_{1}=\check{\pi}\left(\check{U}_{1}\right)$ be the projection of $\check{U}_{1}$ to $\mathbb{T}^{2}$ then $U_{1}=\operatorname{dom}(\mathcal{F})$.

Proof. Remember that $z \in \mathbb{T}^{2}$ is the point such that $\omega_{f}(z)=\alpha_{f}(z)=\mathbb{T}^{2}$. By the proof of the claim above we know that there is a lift $\check{z} \in \check{\pi}^{-1}(z)$ of the point $z \in \mathbb{T}^{2}$ that draws $\check{\Gamma}_{1}$, and moreover $\check{z}$ is non-wandering for $\check{f}$. As the whole $\check{\mathcal{F}}$-transverse trajectory of $\check{z}$, $\check{\gamma}:=\check{I}_{\mathcal{F}}^{\mathbb{Z}}(\check{z})$, has no $\check{\mathcal{F}}$-transverse self intersection then Corollary 4.24 and the remark after it imply that $\check{\gamma}$ is contained in $\check{U}_{1}$. So, by projection, we have that the whole $\mathcal{F}$-transverse trajectory of $z, \gamma:=I_{\mathcal{F}}^{\mathbb{Z}}(z)$ is contained in $U_{1}$.

But, by Lemma 4.23 we know that a whole $\mathcal{F}$-transverse trajectory of a transitive point meets all leafs of $\operatorname{dom}(\mathcal{F})$.

So, we must have that $U_{1}=\operatorname{dom}(\mathcal{F})$.

This statement implies that the frontier $\partial U_{1}$ of $U_{1}$ is contained in the singularity set $\operatorname{sing}(\mathcal{F})$. But more than that, we have $\partial U_{1}=\operatorname{sing}(\mathcal{F})$ because the whole orbit of $z$ is contained in $U_{1}$, and because the orbit of $z$ is dense in $\mathbb{T}^{2}$.

Moreover, by Proposition 5.3 we have that $\check{U}_{1}$ is disjoint from its integer translations and so $U_{1}$ is an inessential set. Therefore, as $U_{1}=\operatorname{dom}(\mathcal{F})$, we have that $\partial U_{1}=\operatorname{sing}(\mathcal{F})$ is a totally essential set. 
Thus, we have that the frontier of the lift $\check{U}_{1}$ is such that $\partial \check{U}_{1} \subset \operatorname{sing}(\check{\mathcal{F}})$. Therefore $\check{U}_{1}$ is an $\check{f}$-invariant set, which means $\check{f}\left(\check{U}_{1}\right)=\check{U}_{1}$. So, for every $\check{z}^{\prime} \in \check{U}_{1}$ its $\alpha$-limit and $\omega$-limit sets are contained in $\check{\check{U}_{1}}=\check{U}_{1} \cup \partial \check{U}_{1}$. Moreover:

Claim 5.10. If $\check{z} \in \check{\pi}^{-1}(z) \cap \check{U}_{1}$, where $z \in \mathbb{T}^{2}$ is the point such that $\omega_{f}(z)=\alpha_{f}(z)=\mathbb{T}^{2}$, then $\alpha_{\check{f}}(\check{z})=\omega_{\check{f}}(\check{z})=\overline{\check{U}_{1}}$.

Proof. It remains to prove that the $\alpha$-limit and $\omega$-limit sets of $\check{z}$ contains $\overline{\check{U}_{1}}$.

We will prove for the case of the $\omega$-limit, the case of the $\alpha$-limit set is analogous.

Suppose, by contradiction, there exists $\check{z}^{\prime} \in \check{U}_{1}$ such that $\check{z}^{\prime} \notin \omega_{\check{f}}(\check{z})$. Let $z^{\prime}$ be the projection of $z^{\prime}$ to $\mathbb{T}^{2}$, as $\omega_{f}(z)=\mathbb{T}^{2}$, we know there exists an increasing sequence of integers $\left(m_{k}\right)_{k \in \mathbb{N}}$ such that $f^{m_{k}}(z) \stackrel{k \rightarrow \infty}{\longrightarrow} z^{\prime}$, this means there exists $k_{0} \geq 1$ large enough such that for every $k>k_{0}$

$$
d_{\mathbb{T}^{2}}\left(f^{m_{k}}(z), z^{\prime}\right)<1 / k
$$

But we are assuming that $\check{z}^{\prime} \notin \omega_{\check{f}}(\check{z})$ then the property 5.15 implies that for every $k>k_{0}$ there must exist $p_{k} \in \mathbb{Z}^{2} \backslash\{(0,0)\}$ such that

$$
d\left(\check{f}^{m_{k}}(\check{z}), \check{z}^{\prime}+p_{k}\right)<1 / k
$$

But this is impossible. Because $\check{U}_{1}$ is $\check{f}$-invariant and does not intersect any of its integer vector translate. Therefore $\check{z}^{\prime} \in \omega_{\check{f}}(\check{z})$.

To conclude, as $\omega$-limit set of $\check{z}$ is a closed set that contains $\check{U}_{1}$, it must to contain its closure. Therefore $\overline{\check{U}_{1}} \subset \omega_{\check{f}}(\check{z})$.

It follows from the proof of Lemma 5.2 that there exists at least one singularity of the foliation $\check{\mathcal{F}}$ that is contained in $\mathrm{B}_{\mathrm{c}}\left(\check{U}_{1}\right)$, we will denote it by $\check{z}^{\prime}$.

Let us consider the annulus $A=\mathbb{R}^{2} \backslash\left\{\check{z}^{\prime}\right\}$. As $\check{z}^{\prime} \in \operatorname{sing}(\check{\mathcal{F}})$, the restriction $\left.\check{f}\right|_{A}$ is well defined and is a homeomorphism isotopic to identity, where $\left.\check{I}\right|_{A}$ and $\left.\check{\mathcal{F}}\right|_{A}$ are a maximal identity isotopy for $\left.\check{f}\right|_{A}$ and a transverse foliation to $\left.\check{I}\right|_{A}$ of $A$, respectively.

As $\check{z}^{\prime} \in \mathrm{B}_{\mathrm{c}}\left(\check{U}_{1}\right) \subset \mathrm{B}_{\mathrm{c}}\left(\check{\Gamma}_{1}\right)$, we have that $\check{\Gamma}_{1}$ is an essential loop in $A$ then $\check{z}_{1} \in \operatorname{ne}^{+}\left(\check{f}_{A}\right)$ and $\operatorname{rot}\left(\check{z}_{1}, \check{f}_{A}\right)$ is a rational number non-zero.

Furthermore, as $\operatorname{sing}(\check{\mathcal{F}})$ is the lift of a totally essential set of $\mathbb{T}^{2}$ we have that there exists $\check{z}_{2} \in \partial \check{U}_{1} \backslash\left\{\check{z}^{\prime}\right\}$ which implies that $\check{z}_{2} \in \operatorname{sing}\left(\check{\mathcal{F}}_{A}\right)=\operatorname{fix}\left(\check{I}_{A}\right)$ and then we have that $\check{z}_{2} \in \operatorname{ne}^{+}\left(\check{f}_{A}\right)$ and $\operatorname{rot}\left(\check{z}_{2}, \check{f}_{A}\right)=0$.

By Claim 5.10 we have $\check{z} \in \operatorname{ne}^{+}\left(\check{f}_{A}\right)$ and furthermore $\check{z}_{1}, \check{z}_{2} \in \omega_{\check{f}_{A}}(\check{z})$. However we have that $\operatorname{rot}\left(\check{z}_{1}, \check{f}_{A}\right) \neq \operatorname{rot}\left(\check{z}_{2}, \check{f}_{A}\right)$ then the contrapositive of Theorem 3.9 implies that $\check{f}_{A}$ possesses a topological horseshoe $\check{\Delta}$ as defined in the introduction. And, as we have that $\check{f}=\check{f}_{A}$ on $\mathbb{R}^{2} \backslash\left\{\check{z}^{\prime}\right\}$ and $\check{f}\left(\check{z}^{\prime}\right)=z^{\prime}$ then $\check{\Delta}$ is also a topological horseshoe for $\check{f}$. And, therefore, by Remark 2.6, we have that $\Delta=\check{\pi}(\check{\Delta})$ is a topological horseshoe for $f$, as in Definition 1.2. 


\subsubsection{Essential loop on $\mathbb{T}^{2}$}

We have $z_{0} \in \operatorname{fix}(f), z_{1} \in \operatorname{fix}\left(f^{q}\right)$ with $q>1$ and $z \in \mathbb{T}^{2}$ the point such that $\omega_{f}(z)=\alpha_{f}(z)=\mathbb{T}^{2}, I$ a maximal isotopy such that $z_{0} \in \operatorname{fix}(I)$ and $\mathcal{F}$ is a transverse foliation to $I$. And now we will suppose that $I^{q_{1}}\left(z_{1}\right)$ is an essential loop.

As before, let $\check{I}=\left(\check{f}_{t}\right)_{t \in[0,1]}$ be the identity isotopy that lifts $I$ to $\mathbb{R}^{2}, \check{f}:=\check{f}_{1}$ the lift of $f$ to $\mathbb{R}^{2}$ given by the isotopy $I$ and $\check{\mathcal{F}}$ foliation of $\mathbb{R}^{2}$ that lifts $\mathcal{F}$. And as $I^{q_{1}}\left(z_{1}\right)$ is an essential loop on $\mathbb{T}^{2}$, there exists $\left(p_{1}, p_{2}\right) \in \mathbb{Z}^{2} \backslash\{(0,0)\}$ such that

$$
\check{f}^{q}\left(\check{z}_{1}\right)=\check{z}_{1}+\left(p_{1}, p_{2}\right), \quad \forall \check{z}_{1} \in \check{\pi}^{-1}\left(z_{1}\right) .
$$

Moreover, $z_{0} \in \operatorname{fix}(I)$ implies $\check{f}\left(\check{z}_{0}\right)=\check{z}_{0}, \forall \check{z}_{0} \in \check{\pi}^{-1}\left(z_{0}\right)$.

This implies that $\rho\left(z_{0}, \check{f}\right)=(0,0)$ and $\rho\left(z_{1}, \check{f}\right)=\frac{1}{q}\left(p_{1}, p_{2}\right)$. And as $\left(p_{1}, p_{2}\right) \neq(0,0)$ we must have that $\rho(\check{f})$ has non-empty interior or is a non-degenerate line segment of rational slope.

a) If $\operatorname{int}(\rho(\check{f})) \neq \emptyset$ then, due to the techniques developed by Llibre and Mackay in [LM91], we already know that $f$ has a topological horseshoe.

Or, by Forcing Theory, Le Calvez and Tal show that (see the Proof of Theorem 64, page 82, in [LCT18a]) in this case there are two $\mathcal{F}$-transverse loops associated to periodic points that have an $\mathcal{F}$-transverse intersection, and by Lemma 4.27 we know that this implies the existence of an admissible loop $\Gamma$ with an $\mathcal{F}$-transverse self-intersection. And then we can apply Theorem 4.28 and get the result.

b) If $\rho(\check{f})$ is a segment of rational slope containing $(0,0)$ and $\frac{1}{q}\left(p_{1}, p_{2}\right)$, and moreover these vectors are realized by $z_{0}$ and $z_{1}$, respectively, then Proposition 5.4 implies that $f$ has a topological horseshoe.

Finishing the proof of Theorem A.

\subsection{Proof of Proposition C}

Now we will prove the following:

Proposition C. Let $f \in \operatorname{Homeo}\left(\mathbb{T}^{2}\right)$ be a topologically transitive homeomorphism with a non-empty fixed point set and such that some power $g=f^{k}$ of $f$, where $k>1$, is isotopic to identity, but $f$ itself is not. If $f$ does not have a topological horseshoe, then for some lift $\check{g} \in \operatorname{Homeo}\left(\mathbb{R}^{2}\right)$ of $g$, the rotation set of $\check{g}$ is equal to $\{(0,0)\}$.

Proof. By assumption we have that $f$ has a fixed point and has no topological horseshoe, and there is $k>1$ such that $g=f^{k}$ is isotopic to identity. So $g=f^{k}$ has a fixed point and has no topological horseshoe. Moreover we can take $\check{g}$ as the lift of $g$ that has a fixed point. Thus $\rho(\check{g})$ contains the origin. 
Suppose, by contradiction, that $\rho(\check{g}) \neq\{(0,0)\}$. Then we have 2 possibilities:

a) $\rho(\check{g})$ has non-empty interior, or

b) $\rho(\check{g})$ is a non-degenerate line segment,

both containing the origin.

Well, we already know that item (a) implies that $g$ has a topological horseshoe, which in turn implies that $f$ also have a topological horseshoe, which is a contradiction to the hypothesis.

Let us analyze item (b): $\rho(\check{g})$ is a non-degenerate line segment containing the origin.

As explained in Chapter 2 (see Proposition 2.10), given an $f \in \operatorname{Homeo}\left(\mathbb{T}^{2}\right)$, there is some linear automorphism $A \in \operatorname{GL}(2, Z)$ such that if $\check{f} \in \operatorname{Homeo}\left(\mathbb{R}^{2}\right)$ is a lift of $f$ then

$$
\check{f}\left(\check{z}+\left(p_{1}, p_{2}\right)\right)=\check{f}(\check{z})+A\left(\begin{array}{c}
p_{1} \\
p_{2}
\end{array}\right) .
$$

So, as $g$ is isotopic to identity, Lemma 3.2 implies that

$$
\rho(\check{g})=\rho\left(\check{f} \circ \check{g} \circ \check{f}^{-1}\right)=A \rho(\check{g}) \text {. }
$$

Let $v \in \rho(\check{g}) \backslash\{(0,0)\}$. So, by (5.17) we have that $A v \in \rho(\check{g})$. As we are assuming that $\rho(\check{g})$ is a non-degenerate line segment containing the origin and $v \neq(0,0)$, then there is some $\lambda \in \mathbb{R}$ such that $A v=\lambda v$. Which means that $v$ is an eigenvector of $A$ associated to the real eigenvalue $\lambda$.

As $g=f^{k}$ is isotopic to identity, thus $A^{k}=\operatorname{Id}$ and therefore $\lambda^{k} v=A^{k} v=v$. So $\lambda$ is a real number such that $\lambda^{k}=1$. Consequently, $\lambda= \pm 1$.

Claim. The slope of the rotation set $\rho(\check{g})$ is rational.

Proof of claim. Indeed, write $v \in \rho(\check{g}) \backslash\{(0,0)\}$ as $v=\left(v_{1}, v_{2}\right)$.Also write $A \in \operatorname{GL}(2, \mathbb{Z})$ as $A=\left(\begin{array}{ll}p_{1} & p_{2} \\ q_{1} & q_{2}\end{array}\right)$.

If $\lambda=1$ then $A v=v$ and

$$
\left\{\begin{array}{l}
p_{1} v_{1}+p_{2} v_{2}=v_{1} \\
q_{1} v_{1}+q_{2} v_{2}=v_{2}
\end{array} \quad \Rightarrow \text { either } v_{1}=0 \text { or } \frac{v_{2}}{v_{1}} \in \mathbb{Q}\right.
$$

If $\lambda=-1$ then $A v=-v$ and

$$
\left\{\begin{array}{l}
p_{1} v_{1}+p_{2} v_{2}=-v_{1} \\
q_{1} v_{1}+q_{2} v_{2}=-v_{2}
\end{array} \quad \Rightarrow \text { either } v_{1}=0 \text { or } \frac{v_{2}}{v_{1}} \in \mathbb{Q}\right.
$$

As $(0,0)$ and $\left(v_{1}, v_{2}\right)$ are in $\rho(\check{g})$ and either $v_{1}=0$ or $\frac{v_{2}}{v_{1}} \in \mathbb{Q}$, we have that the direction of $\rho(\check{g})$ is a multiple of a rational vector. Therefore the slope of $\rho(\check{g})$ must be rational. 
End of the proof of Proposition C. By Proposition 5.4 we have that in this situation $f$ would have a topological horseshoe, which is a contradiction with hypothesis.

Therefore $\rho(\check{g})$ must be equal to $\{(0,0)\}$, proving the proposition. 


\section{Chapter 6}

\section{Proof of Theorem B}

Our main goal in this chapter is to prove the following result:

Theorem B. Let $f \in \operatorname{Homeo}\left(\mathbb{T}^{2}\right)$ be a topologically transitive homeomorphism with a non-empty fixed point set and such that a power $f^{k}$ of $f$, where $k \geq 1$, is isotopic to a Dehn Twist. Then $f$ has a topological horseshoe.

Let us first introduce some fundamental concepts about homeomorphisms isotopic to Dehn Twist.

As presented at Chapter 2 if $f \in \operatorname{Homeo}\left(\mathbb{T}^{2}\right)$ is isotopic to a Dehn Twist and if $\check{f} \in \operatorname{Homeo}\left(\mathbb{R}^{2}\right)$ is a lift of $f$ then there is some $A \in \operatorname{SL}(2, \mathbb{Z})$ that is conjugate to matrix $\left(\begin{array}{cc}1 & m \\ 0 & 1\end{array}\right)$ where $m \in \mathbb{Z} \backslash\{0,0\}$ and such that for all $\left(p_{1}, p_{2}\right) \in \mathbb{Z}^{2}$

$$
\check{f}\left(\check{z}+\left(p_{1}, p_{2}\right)\right)=\check{f}(\check{z})+A\left(\begin{array}{c}
p_{1} \\
p_{2}
\end{array}\right) .
$$

As the proofs that we will presented in this chapter are preserved by conjugation, we will consider only the case where $A$ is in this special format. So

$$
A=\left(\begin{array}{cc}
1 & m \\
0 & 1
\end{array}\right)
$$

where $m \in \mathbb{Z} \backslash\{0\}$. And the induced map by $A$ in $\mathbb{T}^{2}$ we will denote it by $f_{A}$.

If we observe the matrix $A \in \mathrm{SL}(2, \mathbb{Z})$ we will notice that any lift map $\check{f} \in \operatorname{Homeo}\left(\mathbb{R}^{2}\right)$ of $f$ commutes with the horizontal translations, but this is not true for vertical translations. In fact, if we translate the vector $\check{z} \in \mathbb{R}^{2}$ vertically $p \in \mathbb{Z}$ times, $\check{z}+(0, p)$, then

$$
\check{f}(\check{z}+(0, p))=(\check{f}(\check{z})+(0, p))+(m p, 0) .
$$

Which means that if we translate the vector vertically then its image is translated vertically and horizontally. Moreover the higher the vector, the greater its horizontal translation (in norm). 
Now, suppose that $\check{f}$ has a fixed point $\check{z} \in \mathbb{R}^{2}$, then there exist $\check{z} \in \mathbb{R}^{2}$ such that

$$
\check{f}(\check{z})=\check{z},
$$

but if we translate the vector vertically $p \in \mathbb{Z}$ times then

$$
\check{f}(\check{z}+(0, p))=\check{z}+(0, p)+(m p, 0) .
$$

Therefore, the rotation vector of the fixed point $\check{\pi}(\check{z}) \in \mathbb{T}^{2}$ is not well-defined independently of the choice of the lift. Thus, it does not make sense to define a bi-dimensional rotation set for homeomorphism isotopic to a Dehn twist. Instead, it is considered the vertical annulus $\mathbb{A}=\mathbb{T}^{1} \times \mathbb{R}$ and it is defined a vertical rotation set in this covering space of the $\mathbb{T}^{2}$.

As we establish in the Notation 2.4, let $\check{\pi}: \mathbb{R}^{2} \rightarrow \mathbb{T}^{2}$ be the canonical universal covering of $\mathbb{T}^{2}$ and $\check{\tau}: \mathbb{R}^{2} \rightarrow \mathbb{A}$ the canonical universal covering of $\mathbb{A}$ and $\hat{\pi}: A \rightarrow \mathbb{T}^{2}$ a covering map such that $\hat{\pi} \circ \check{\tau}=\check{\pi}$.

As defined in [AZ02] and also in [Doe97, the following definition of vertical rotation set is analogous to definition for rotation set for homeomorphism isotopic to identity, given by to Misiurewicz and Ziemian in [MZ89.

Definition 6.1. Let $f: \mathbb{T}^{2} \rightarrow \mathbb{T}^{2}$ be a homeomorphism isotopic to a Dehn twist and fix a lift $\hat{f}: \mathbb{A} \rightarrow \mathbb{A}$ of $f$ to $\mathbb{A}$. We define the vertical rotation set of the lift $\hat{f}$, namely $\rho_{V}(\hat{f})$, as the following

$$
\rho_{V}(\hat{f})=\bigcap_{i \geq 1} \overline{\bigcup_{n \geq i}\left\{\frac{p_{2}\left(\hat{f}^{n}(\hat{z})-\hat{z}\right)}{n}: \hat{z} \in \mathbb{A}\right\}} .
$$

If there is $\hat{z} \in \mathbb{A}$ such that the limit

$$
\lim _{n \rightarrow \infty} \frac{\mathrm{p}_{2}\left(\hat{f}^{n}(\hat{z})-\hat{z}\right)}{n} \text { exists }
$$

then we will say that $\hat{z} \in \mathbb{A}$ has a vertical rotation number and we will denote it by $\rho_{V}(\hat{z}, \hat{f})$. Furthermore, observe that the restriction to the second coordinate implies that for all $p \in \mathbb{Z}$,

$$
\rho_{V}(\hat{z}+(0, p), \hat{f})=\rho_{V}(\hat{z}, \hat{f})
$$

whenever $\hat{z} \in \mathbb{A}$ has a vertical rotation number. Moreover, this implies that it only depends of the point $z=\hat{\pi}(\hat{z}) \in \mathbb{T}^{2}$. So, we can use de notation $\rho_{V}(z, \hat{f})$, where $z \in \mathbb{T}^{2}$, instead.

Regarding the dependence of the lift, the vertical rotation set preserves some good properties, as in the uni-dimensional case:

Proposition 6.2. Let $f \in \operatorname{Homeo}\left(\mathbb{T}^{2}\right)$ be isotopic to $f_{A}$ and fix a lift $\hat{f} \in \operatorname{Homeo}(\mathbb{A})$ of f. Then, for all $p \in \mathbb{Z}$ and $q \in \mathbb{N}$,

1. $\rho_{V}(\hat{f})$ is a non-empty and compact interval of $\mathbb{R}$; 
2. $\rho_{V}\left(\hat{f}^{q}-(0, p)\right)=q \rho_{V}(\hat{f})-p$;

3. $\rho_{V}\left(\hat{f}^{-1}\right)=-\rho_{V}(\hat{f})$.

For other properties, see AZ02 and AZ05.

As before, we can define the vertical rotation number associated to an invariant measure.

Definition 6.3. Let $f \in \operatorname{Homeo}\left(\mathbb{T}^{2}\right)$ be isotopic to a Dehn twist and fix a lift $\hat{f} \in$ $\operatorname{Homeo}(\mathbb{A})$ of $f$. For an $f$-invariant Borel probability measure $\mu$, the vertical rotation number associated to $\mu$ is defined as

$$
\rho_{V}(\mu)=\int_{\mathbb{T}^{2}} \varphi \mathrm{d} \mu
$$

where the vertical displacement function $\varphi: \mathbb{T}^{2} \rightarrow \mathbb{T}^{1} \times \mathbb{R}$ is given by $\varphi(z)=\mathrm{p}_{2}(\hat{f}(\hat{z})-\hat{z})$, where $\hat{z} \in \hat{\pi}^{-1}(z)$. Note that this definition does not depend on the choice of $\hat{z}$, only of $z \in \mathbb{T}^{2}$.

By Birkhoff's Ergodic Theorem we have that if $\mu$ is an $f$-ergodic Borel probability measure such that $\rho_{V}(\mu)=a$ then for $\mu$-almost every point $z \in \mathbb{T}^{2}$ and any $\hat{z} \in \hat{\pi}^{-1}(z)$,

$$
\lim _{n \rightarrow \infty} \frac{\hat{f}^{n}(\hat{z})-\hat{z}}{n}=a
$$

As $\rho_{V}$, in Definition 6.3, is a continuous linear functional on $\mathcal{M}_{\mathbb{T}^{2}}(f)$, the set of the $f$ -

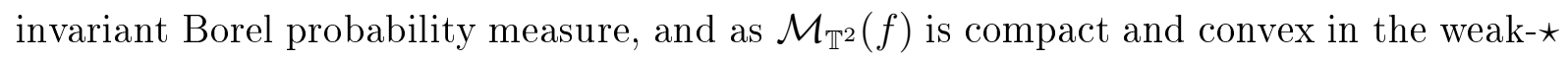
topology we must have that

$$
\rho_{V}\left(\mathcal{M}_{\mathbb{T}^{2}}(f)\right)=[a, b] \subset \mathbb{R}
$$

where $a=\inf _{\mu \in \mathcal{M}_{\mathbb{T}^{2}}(f)} \rho_{V}(\mu)$ and $b=\sup _{\mu \in \mathcal{M}_{\mathbb{T}^{2}}(f)} \rho_{V}(\mu)$, and it is possible that $a=b$.

And moreover, is possible to show that $\rho_{V}(\hat{f})=\rho_{V}\left(\mathcal{M}_{\mathbb{T}^{2}}(f)\right)=[a, b]$. Thus, using the fact that $\mathcal{M}_{\mathbb{T}^{2}}(f)$ is compact and convex and its extremal points are ergodic measures we get the next proposition:

Proposition 6.4. There exist two f-ergodic Borel probability measure $\mu_{a}$ and $\mu_{b}$ on $\mathbb{T}^{2}$ such that $\rho_{V}\left(\mu_{a}\right)=a$ and $\rho_{V}\left(\mu_{b}\right)=b$.

And then, by Birkhoff's Ergodic Theorem there are points $\hat{z}_{a}, \hat{z}_{b} \in \mathbb{A}$ such that $\rho_{V}\left(\hat{f}, \hat{z}_{a}\right)=a$ and $\rho_{V}\left(\hat{f}, \hat{z}_{b}\right)=b$.

Before proving Theorem B, we state a result due to Addas-Zanata, Tal and Garcia which assure that if $\rho_{V}(\hat{f})=\{0\}$ then there exists $\hat{K} \subset \mathbb{A}$ an essential $\hat{f}$-invariant continuum. 
Theorem 6.5 (Theorem 2 in AZTG12]). Given $f \in \operatorname{Homeo}\left(\mathbb{T}^{2}\right)$ isotopic to an $f_{A}$ and a lift $\hat{f} \in \operatorname{Homeo}(\mathbb{A})$, if $\rho_{V}(\hat{f})=\left\{\frac{p}{q}\right\}$ for some $p \in \mathbb{Z}$ and $q \in \mathbb{N}$, then there exists a compact connected set $\hat{K} \subset \mathbb{A}$, invariant under $\hat{f}^{q}-(0, p)$, which separates the ends of the vertical annulus.

Now, we are able to show Theorem B.

Proof of Theorem B. Let $g=f^{k}$, where $k \geq 1$, be the positive power of $f$ that is isotopic to a Dehn twist. So, there is a matrix $A \in \mathrm{SL}(2, \mathbb{Z})$

$$
A=\left(\begin{array}{cc}
1 & m \\
0 & 1
\end{array}\right)
$$

where $m \in \mathbb{Z} \backslash\{(0,0)\}$ and such that $g$ is conjugate to a homeomorphisms isotopic to the Dehn twist map induced by the matrix $A$, and we will assume for simplicity that $g$ is itself isotopic to $f_{A}$. This means that if $\check{g} \in \operatorname{Homeo}\left(\mathbb{R}^{2}\right)$ is a lift of $g$ to $\mathbb{R}^{2}$ then

$$
\check{g}\left(\check{z}+\left(p_{1}, p_{2}\right)\right)=\check{g}(\check{z})+A\left(\begin{array}{c}
p_{1} \\
p_{2}
\end{array}\right) .
$$

Since $f$ has a fixed point, so does $g$. Thus, let $\check{g} \in \operatorname{Homeo}\left(\mathbb{R}^{2}\right)$ be the lift of $g$ that has a fixed point. We have that $\check{g}$ induces a lift $\hat{g} \in \operatorname{Homeo}(\mathbb{A})$ of $g$ to $\mathbb{A}=\mathbb{T}^{1} \times \mathbb{R}$ such that the following diagram commutes:

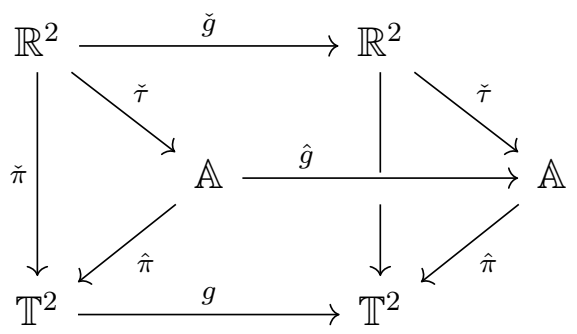

Take this homeomorphism $\hat{g} \in \operatorname{Homeo}(\mathbb{A})$ induced by $\check{g}$, and let $\rho_{V}(\hat{g})$ be its vertical rotation interval. In addition, as $\check{g}$ has a fixed point then $\hat{g}$ also has. Therefore $0 \in \rho_{V}(\hat{g})$.

So, we have two possibilities:

a) $\rho_{V}(\hat{g})=\{0\}$ or,

b) $\rho_{V}(\hat{g})$ is a non-degenerate interval of $\mathbb{R}$ containing $\{0\}$.

\subsection{If $\rho_{V}(\hat{g})=\{0\}$}

In this case, by Theorem 6.5, there exists $\hat{K} \subset \mathbb{A}$ an essential $\hat{g}$-invariant continuum. So, there exists an integer $M_{0}>0$ such that $\hat{K} \subset \mathbb{T}^{1} \times\left[-M_{0}, M_{0}\right]$. Let $\hat{K}^{-}$and $\hat{K}^{+}$ be the connected components of $(\mathbb{A}) \backslash \hat{K}$ that contains $-\infty$ and $+\infty$, respectively. As $\hat{K}$ is $\hat{g}$-invariant, then $\hat{K}^{-}$and $\hat{K}^{+}$are also $\hat{g}$-invariant. Then, there is $M>M_{0}$ such that 
$\hat{K}^{-} \cap\left(\hat{K}^{+}-(0, M)\right)$ is an open, connected and $\hat{g}$-invariant set that contains a fundamental domain of $\mathbb{T}^{2}$. See Figure 6.1 .

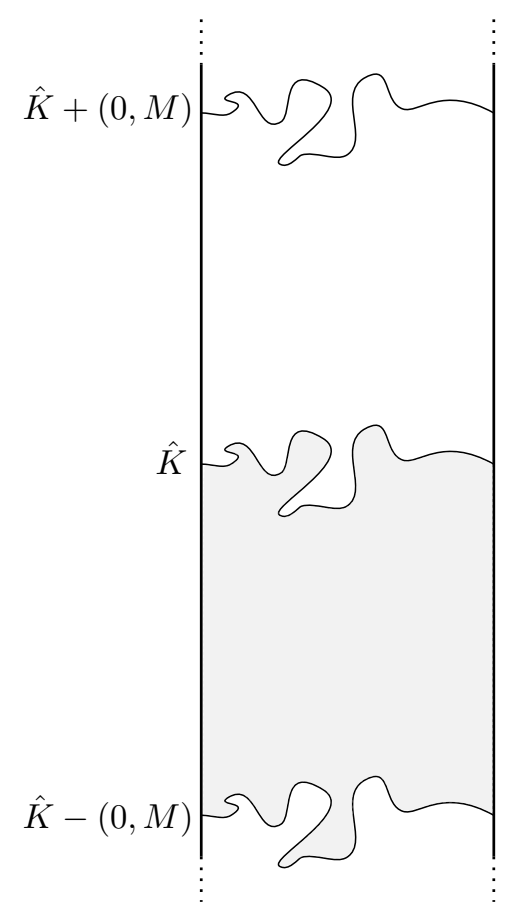

Figure 6.1: Existence of a continuum $\hat{K}$.

Therefore, for all $\hat{w} \in \mathbb{A}$ and all integer $n>0$ there is some $M^{\prime}>M$ such that

$$
\left|\mathrm{p}_{2}\left(\hat{g}^{n}(\hat{w})-\hat{w}\right)\right|<M^{\prime}
$$

As observed in the proof of Proposition 5.4 this means that for all points $\hat{w} \in \mathbb{A}$, the $\omega_{\hat{g}}(\hat{w})$ is not empty.

As $g \in \operatorname{Homeo}\left(\mathbb{T}^{2}\right)$ is isotopic to Dehn Twist and the diagram in 6.2 commutes, we have that $\hat{g}(\hat{w}+(0, p))=\hat{g}(\hat{w})+(0, p)$, for all $p \in \mathbb{Z}$.

Indeed, we know that $\check{g}(\check{w}+(0, p))=\check{g}(\check{w})+(m, p)$, for all $\check{w} \in \mathbb{R}^{2}$. Thus, if $\check{w}+(0, p) \in$ $\check{\tau}^{-1}(\hat{w}+(0, p))$ then

$$
\begin{aligned}
\hat{g}(\hat{w}+(0, p)) & =\hat{g}(\check{\tau}(\check{w}+(0, p))) \\
& =\check{\tau}(\check{g}(\check{w}+(0, p))) \\
& =\check{\tau}(\check{g}(\check{w})+(m, p)) \\
& =\check{\tau}(\check{g}(\check{w}))+(0, p) \\
& =\hat{g}(\hat{w})+(0, p), \text { where } \hat{w}=\check{\tau}(\check{w})
\end{aligned}
$$

Since $\check{g} \in \operatorname{Homeo}\left(\mathbb{R}^{2}\right)$ commutes with the integer horizontal translations then homotopy theory implies that $\hat{g} \in \operatorname{Homeo}(\mathbb{A})$ is homotopic to identity. Moreover $\hat{g}$ preserves the ends of $\mathbb{A}$. Thus, by [BCLR20] (see propositions 3.3, 3.6 and 8.1), $\hat{g}$ is isotopic to identity. 
Moreover, as $f$ is topologically transitive, the Corollary 5.6 implies that $\hat{g}$ is nonwandering.

As we did in the proof of Proposition 5.4, if we suppose, by contradiction, that $f$ has no topological horseshoe, then $g$ and also $\hat{g}$ have no topological horseshoe. And moreover, the $\operatorname{map}_{\check{g}}: \mathbb{A} \rightarrow \mathbb{R}$ is well-defined, continuous and must to be constant by Lemma 5.5 and Remark 5.7. However this is impossible. Let us explain why.

We know that $\hat{g} \in \operatorname{Homeo}(\mathbb{A})$ is a lift of the map $g \in \operatorname{Homeo}\left(\mathbb{T}^{2}\right)$ that is isotopic to a Dehn twist map induced by

$$
A=\left(\begin{array}{cc}
1 & m \\
0 & 1
\end{array}\right),
$$

thus if $\check{g} \in \operatorname{Homeo}\left(\mathbb{R}^{2}\right)$ is a lift of $g \in \operatorname{Homeo}\left(\mathbb{T}^{2}\right)$ then $\check{g}$ is also a lift of $\hat{g} \in \operatorname{Homeo}(\mathbb{A})$ and

$$
\check{g}\left(\check{z}+\left(p_{1}, p_{2}\right)\right)=\check{g}(\check{z})+\left(p_{1}+m p_{2}, p_{2}\right) \text { where }\left(p_{1}, p_{2}\right) \in \mathbb{Z}^{2} \text {. }
$$

Moreover, we know that there is $\hat{z}_{0} \in \mathbb{A}$ such that

$$
\hat{g}\left(\hat{z}_{0}\right)=\hat{z}_{0}
$$

then we can assume that $\operatorname{rot}\left(\hat{z}_{0}, \breve{g}\right)=0$, because if it is not then we can take some horizontal integer translation of $\check{g}$ that satisfies this property. And, because of (6.4), if we take $\hat{z}_{1}=\hat{z}_{0}+(0,1)$ then

$$
\check{g}\left(\check{z}_{1}\right)=\check{z}_{1}+(m, 0), \check{z}_{1} \in \tau^{-1}\left(\hat{z}_{1}\right)
$$

and then $\operatorname{rot}\left(\hat{z}_{1}, \check{g}\right)=m \neq 0$. And this is a contradiction with the fact that $\operatorname{rot}_{\check{g}}$ is a constant map.

So, for this case, we prove, by contradiction, that $f$ must to have a topological horseshoe.

\subsection{If $\rho_{V}(\hat{g})$ is a non-degenerate compact interval of $\mathbb{R}$ containing $\{0\}$}

The existence of a horseshoe for this case is already known, althought we could not find it explicitly written in the literature. In any case it follows as a scholium of Theorem 3.1 due to Doeff and Misiurewicz in [DM97 together with the techniques developed by Llibre and Mackay in [LM91. Furthermore, the case where $f$ is a $C^{1+\epsilon}$ diffeomorphism has already been proved by Addas-Zanata in [AZ15.

In the following we will give a new proof for the existence of a topological horseshoe for this case, only using Forcing Theory and Rotation Theory.

A result in rotation theory that we will use here is Theorem 1 in AZ05] due to 
Addas-Zanata.

Definition 6.6. Let $\hat{h} \in \operatorname{Homeo}(\mathbb{A})$ be isotopic to the identity and let $\check{h} \in \operatorname{Homeo}\left(\mathbb{R}^{2}\right)$ be one of its lifts. We say that:

1. $\hat{h}$ satisfies the infinity twist condition (ITC) if for any lift $\check{h} \in \operatorname{Homeo}\left(\mathbb{R}^{2}\right)$ the following property holds:

$$
\mathrm{p}_{1} \circ \check{h}(\check{x}, \check{y}) \rightarrow \pm \infty \quad \text { as } \check{y} \rightarrow \pm \infty
$$

2. $(\hat{h}, \check{h})$ has a periodic orbit of rotation number $\frac{p}{q}$, if there exists $\hat{z} \in \mathbb{A}$ such that $\hat{h}^{q}(\hat{z})=\hat{z}$ and $\check{h}^{q}(\check{z})=\check{z}+(p, 0)$ for all $\check{z} \in \check{\tau}^{-1}(\hat{z})$.

Note that if $\hat{h} \in \operatorname{Homeo}(\mathbb{A})$ is a lift of $h \in \operatorname{Homeo}\left(\mathbb{T}^{2}\right)$ homeomorphism isotopic to $f_{A}$ then $\hat{h}$ is isotopic to identity (as explained before) and satisfies ITC property. Indeed, if $\check{h} \in \operatorname{Homeo}\left(\mathbb{R}^{2}\right)$ is a lift of $\hat{h}$, then $\check{h}$ is also a lift of $h$ satisfying

$$
\check{h}\left(\check{z}+\left(p_{1}, p_{2}\right)\right)=\check{h}(\check{z})+\left(p_{1}+m p_{2}, p_{2}\right),
$$

where $m \geq 1$ is given by the Dehn Twist map.

Theorem 6.7 (Theorem 1 at AZ05]). Let $\hat{h} \in \operatorname{Homeo}(\mathbb{A})$ be isotopic to the identity and let $\breve{h} \in \operatorname{Homeo}\left(\mathbb{R}^{2}\right)$ be some lift of $h$. Suppose $\hat{h}$ satisfies the ITC property and there exist points $\hat{z}_{1}, \hat{z}_{2} \in \mathbb{A}$ such that

$$
\begin{aligned}
& \mathrm{p}_{2} \circ \hat{h}^{n}\left(\hat{z}_{1}\right) \rightarrow \mp \infty \quad \text { as } n \rightarrow \pm \infty \text { and } \\
& \mathrm{p}_{2} \circ \hat{h}^{n}\left(\hat{z}_{2}\right) \rightarrow \pm \infty \quad \text { as } n \rightarrow \pm \infty
\end{aligned}
$$

then for all rational numbers $\frac{p}{q},(\hat{h}, \breve{h})$ has at least two periodic orbits with rotation number equal to $\frac{p}{q}$.

Let $g, \check{g}$ and $\hat{g}$ be as in the beginning of the proof of Theorem B. That is $g \in \operatorname{Homeo}\left(\mathbb{T}^{2}\right)$ is isotopic to $f_{A}, \check{g} \in \operatorname{Homeo}\left(\mathbb{R}^{2}\right)$ is the lift of $g$ that has a fixed point and $\hat{g} \in \operatorname{Homeo}(\mathbb{A})$ is a lift of $g$ induced by $\check{g}$ and the diagram in 6.2 commutes. Then, as explained before, we know that $\hat{g} \in \operatorname{Homeo}(\mathbb{A})$ is isotopic to identity and, moreover, $0 \in \rho_{V}(\hat{g})$.

Now, we will assume that $\rho_{V}(\hat{g})$ is a non-degenerate compact interval of $\mathbb{R}$ containing $\{0\}$. We can assume, without loss of generality, that $\rho_{V}(\hat{g})=[a, b]$ where $a<0<b$. Let us explain why.

If $0 \notin \operatorname{int}\left(\rho_{V}(\hat{g})\right)$ then $0 \in \partial\left(\rho_{V}(\hat{g})\right)$. Suppose that $\rho_{V}(\hat{g})=[0, c]$ (the other case, namely $\rho_{V}(\hat{g})=[c, 0]$, is analogous). Take any $\frac{p}{q} \in(0, c)$, where $p \in \mathbb{N}$ and $q \in \mathbb{N}$. So, $h=g^{q}$ is a homeomorphism in $\mathbb{T}^{2}$ isotopic to $f_{A}$ and such the lift $\hat{h}:=\hat{g}^{q}-(0, p)$ to $\mathbb{A}$ has the property that $0 \in \operatorname{int}\left(\rho_{V}(\hat{h})\right)$, once $\rho_{V}(\hat{h})=q \rho_{V}(\hat{g})-p$. 
So, if $0 \notin \operatorname{int}\left(\rho_{V}(\hat{g})\right)$ then there is a power $h=g^{q}$ of $g$ and a lift $\hat{h}=\hat{g}^{q}-p$ to $\mathbb{A}$ such that $0 \in \operatorname{int}\left(\rho_{V}(\hat{h})\right)$ and for the rest of the proof we would have just used $h$ and $\hat{h}$ instead of $g$ and $\hat{g}$.

So, let $\hat{g}$ be such that $\rho_{V}(\hat{g})=[a, b]$, where $a<0<b$. The Proposition 6.4 implies that there exist $z_{a}, z_{b} \in \mathbb{T}^{2}$ such that

$$
\begin{aligned}
& \rho_{V}\left(\hat{g}, z_{a}\right)=a \quad \text { and } \quad \rho_{V}\left(\hat{g}^{-1}, z_{a}\right)=-a \\
& \rho_{V}\left(\hat{g}, z_{b}\right)=b \quad \text { and } \quad \rho_{V}\left(\hat{g}^{-1}, z_{b}\right)=-b
\end{aligned}
$$

Lemma 6.8. Let $z_{a}, z_{b} \in \mathbb{T}^{2}$ be the points that satisfy 6.5. If $\hat{z}_{a} \in \hat{\pi}^{-1}\left(z_{a}\right)$ and $\hat{z}_{b} \in \hat{\pi}^{-1}\left(z_{b}\right)$ then

$$
\begin{aligned}
& \mathrm{p}_{2} \circ \hat{g}^{n}\left(\hat{z}_{a}\right) \rightarrow \mp \infty \quad \text { as } n \rightarrow \pm \infty \text { and } \\
& \mathrm{p}_{2} \circ \hat{g}^{n}\left(\hat{z}_{b}\right) \rightarrow \pm \infty \quad \text { as } n \rightarrow \pm \infty .
\end{aligned}
$$

Proof. By definition, $\rho_{V}\left(\hat{g}, z_{a}\right)=a$ is

$$
\lim _{n \rightarrow+\infty} \frac{\mathrm{p}_{2}\left(\hat{g}^{n}\left(\hat{z}_{a}\right)-\hat{z}_{a}\right)}{n}=a
$$

where $\hat{z}_{a} \in \hat{\pi}^{-1}\left(z_{a}\right)$ is independent of the choice. But, as $\left|\mathrm{p}_{2}\left(\hat{z}_{a}\right)\right|<L$, where $L>0$ is a real number, the above equation is equivalent to

$$
\lim _{n \rightarrow+\infty} \frac{\mathrm{p}_{2}\left(\hat{g}^{n}\left(\hat{z}_{a}\right)\right)}{n}=a<0, \quad \hat{z}_{a} \in \hat{\pi}^{-1}\left(z_{a}\right) .
$$

Then

$$
\lim _{n \rightarrow+\infty} \mathrm{p}_{2}\left(\hat{g}^{n}\left(\hat{z}_{a}\right)\right)=-\infty
$$

Analogously, if $\rho_{V}\left(\hat{g}^{-1}, z_{a}\right)=-a>0$ then

$$
\lim _{n \rightarrow+\infty} \hat{\mathrm{p}}_{2}\left(g^{-n}\left(\hat{z}_{a}\right)\right)=\lim _{n \rightarrow-\infty} \hat{\mathrm{p}}_{2}\left(g^{n}\left(\hat{z}_{a}\right)\right)=+\infty
$$

The proof for $\mathrm{p}_{2} \circ \hat{g}^{n}\left(\hat{z}_{b}\right) \rightarrow \pm \infty$ as $n \rightarrow \pm \infty$ is analogous.

Let $\check{g} \in \operatorname{Homeo}\left(\mathbb{R}^{2}\right)$ be a lift of $\hat{g}$. As $\hat{g}$ is a lift of a homeomorphism isotopic to $f_{A}$ in $\mathbb{T}^{2}$, we have that $\hat{g}$ satisfies ITC. And together with the Lemma 6.8 we have, by Theorem 6.7. that for all rationals $\frac{p}{q} \in[a, b],(\hat{g}, \check{g})$ has a periodic orbit with rotation number $\frac{p}{q}$.

So, there are $\hat{w}, \hat{w}^{\prime} \in \mathbb{A}, p, p^{\prime} \in \mathbb{Z}$ and $q, q^{\prime} \in \mathbb{N}$ such that $\frac{p}{q} \neq \frac{p^{\prime}}{q^{\prime}}$ and

$$
\begin{array}{ll}
\hat{g}^{q}(\hat{w})=\hat{w}, & \check{g}^{q}(\check{w})=\check{w}+(p, 0), \text { where } \check{w} \in \check{\tau}^{-1}(w) \\
\hat{g}^{q^{\prime}}\left(\hat{w}^{\prime}\right)=\hat{w}^{\prime}, & \check{g}^{q^{\prime}}\left(\check{w}^{\prime}\right)=\check{w}^{\prime}+\left(p^{\prime}, 0\right), \text { where } \check{w} \in \check{\tau}^{-1}(w)
\end{array}
$$

This means that $w, w^{\prime} \in \operatorname{ne}^{+}(\hat{g}), \operatorname{rot}(\hat{w}, \check{g})=\frac{p}{q}$ and $\operatorname{rot}\left(\hat{w}^{\prime}, \check{g}\right)=\frac{p^{\prime}}{q^{\prime}}$.

Now, let $\hat{g}_{\text {sphere }}$ be the continuous extension of $\hat{g}$ to $\mathbb{S}^{2}=\mathbb{A} \cup\{N, S\}$, then Lemma 6.8 
implies that

$$
\begin{aligned}
(\omega)_{\hat{g}_{\text {sphere }}}\left(\hat{z}_{a}\right) & =(\alpha)_{\hat{g}_{\text {sphere }}}\left(\hat{z}_{b}\right)=\{S\} \\
(\alpha)_{\hat{g}_{\text {sphere }}}\left(\hat{z}_{a}\right) & =(\omega)_{\hat{g}_{\text {sphere }}}\left(\hat{z}_{b}\right)=\{N\}
\end{aligned}
$$

So, follows that $N$ and $S$ are in the same Birkhoff recurrence classes of $\hat{g}_{\text {sphere }}$.

Therefore, Proposition 3.10 implies that $\hat{g}$ has a topological horseshoe. And, as explained before, it follows that $g$, and therefore $f$, have a topological horseshoe, as we wanted to prove. 


\section{Bibliography}

[AZ02] Salvador Addas-Zanata. On the existence of a new type of periodic and quasiperiodic orbits for twist maps of the torus. Nonlinearity, 15(5):1399, 2002.

[AZ05] Salvador Addas-Zanata. Some extensions of the Poincaré-Birkhoff theorem to the cylinder and a remark on mappings of the torus homotopic to Dehn twists. Nonlinearity, 18(5):2243, 2005.

[AZ15] Salvador Addas-Zanata. Area-preserving diffeomorphisms of the torus whose rotation sets have non-empty interior. Ergodic Theory and Dynamical Systems, $35(1): 1-33,2015$.

[AZTG12] Salvador Addas-Zanata, Fábio Armando Tal and Bráulio Augusto Garcia. Dynamics of homeomorphisms of the torus homotopic to dehn twists. Ergodic Theory and Dynamical Systems, 34(2):409-422, 2012.

[BCLR20] François Béguin, Sylvain Crovisier and Frédéric Le Roux. Fixed point sets of isotopies on surfaces. Journal of the European Mathematical Society, 22(6):1971-2046, 2020.

[BLR20] François Béguin and Frédéric Le Roux. Dynamique topologique sur les surfaces. https://www.math.univ-paris13.fr/ beguin/Enseignement_ files/Cours_1.pdf, (2007) Accessed on October 14th, 2020.

[Bro12] Luitzen Egbertus Jan Brouwer. Beweis des ebenen translationssatzes. Mathematische Annalen, 72(1):37-54, 1912.

[Con18] Jonathan Conejeros. The local rotation set is an interval. Ergodic Theory and Dynamical Systems, 38(7):2571-2617, 2018.

[Dáv18] Pablo Dávalos. On annular maps of the torus and sublinear diffusion. Journal of the Institute of Mathematics of Jussieu, 17(4):913-978, 2018.

[DM97] Erik Doeff and Michał Misiurewicz. Shear rotation numbers. Nonlinearity, 10(6):1755-1762, 1997.

[Doe97] Erik Doeff. Rotation measures for homeomorphisms of the torus homotopic to a Dehn twist. Ergodic Theory and Dynamical Systems, 17(3):575-591, 1997.

[Eps66] David B. A. Epstein. Curves on 2-manifolds and isotopies. Acta Mathematica, 115:83-107, 1966.

[FH13] John Franks and Michael Handel. Entropy zero area preserving diffeomorphisms of $\mathbb{S}^{2}$. Geometry \& Topology, 16(4):2187-2284, 2013. 
[FM90] John Franks and Michał Misiurewicz. Rotation sets of toral flows. Proceedings of the American Mathematical Society, 109(1):243-249, 1990.

[Fra88] John Franks. Recurrence and fixed points of surface homeomorphisms. Ergodic theory and dynamical systems, 8(8*):99-107, 1988.

[Fra89] John Franks. Realizing rotation vectors for torus homeomorphisms. Transactions of the American Mathematical Society, 311(1):107-115, 1989.

[Fra92] John Franks. A new proof of the Brouwer plane translation theorem. Ergodic Theory and Dynamical Systems, 12(2):217-226, 1992.

[Fra95] John Franks. The rotation set and periodic points for torus homeomorphisms. Dynamical Systems and Chaos (Aoki, Shiraiwa, and Takahashi, eds.), World Scientific, Singapore, 41-48, 1995.

[Han92] Michael Handel. There are no minimal homeomorphisms of the multipunctured plane. Ergodic Theory and Dynamical Systems, 12(1):75-83, 1992.

[Hat02] Allen Hatcher. Algebraic topology. Cambridge University Press, Also available online at http: //www. math. cornell. edu/ “hatcher/AT/ATpage.html, 2002.

[Jau14] Olivier Jaulent. Existence d'un feuilletage positivement transverse à un homéomorphisme de surface. Annales de l'institut Fourier, 64(4):1441-1476, 2014.

[Kat80] Anatole Katok. Lyapunov exponents, entropy and periodic orbits for diffeomorphisms. Publications Mathématiques de l'IHÉS, 51:137-173, 1980.

[KH97] Anatole Katok and Boris Hasselblatt. Introduction to the modern theory of dynamical systems, vol. 54. Cambridge University Press, 1997.

[KK08] Alejandro Kocsard and Andres Koropecki. Free curves and periodic points for torus homeomorphisms. Ergodic Theory and Dynamical Systems, 28(6):18951915, 2008.

[KT14] Andres Koropecki and Fábio Armando Tal. Strictly toral dynamics. Inventiones mathematicae, 196(2):339-381, 2014.

[KY01] Judy Kennedy and James Yorke. Topological horseshoes. Transactions of the American Mathematical Society, 353(6):2513-2530, 2001.

[LC04] Patrice Le Calvez. Une version feuilletée du théoreme de translation de Brouwer. Commentarii mathematici helvetici, 79(2):229-259, 2004.

[LC05] Patrice Le Calvez. Une version feuilletée équivariante du théoreme de translation de Brouwer. Publications Mathématiques de l'IHÉS, 102:1-98, 2005.

[LCT18a] Patrice Le Calvez and Fábio Armando Tal. Forcing theory for transverse trajectories of surface homeomorphisms. Inventiones mathematicae, 212(2):619$729,2018$. 
[LCT18b] Patrice Le Calvez and Fábio Armando Tal. Topological horseshoes for surface homeomorphisms. arXiv e-print at https: // arxiv.org/abs/1803. $04557 v 2,2018$.

[LM91] Jaume Llibre and Robert S MacKay. Rotation vectors and entropy for homeomorphisms of the torus isotopic to the identity. Ergodic Theory and Dynamical Systems, 11(1):115-128, 1991.

[LR13] Frédéric Le Roux. L'ensemble de rotation autour d'un point fixe. Astérisque, Soc. Math. de France, 350:1-109, 2013.

[MZ89] Michał Misiurewicz and Krystyna Ziemian. Rotation sets for maps of tori. Journal of the London Mathematical Society, 2(3):490-506, 1989.

[Poi52] Henri Poincaré. Oeuvres completes. tome 1. Gauthier-Villars, Paris, 137-158, 1952.

[Ree81] Mary Rees. A minimal positive entropy homeomorphism of the 2-torus. Journal of the London Mathematical Society, 2(3):537-550, 1981.

[Sma67] Stephen Smale. Differentiable dynamical systems. Bulletin of the American mathematical Society, 73(6):747-817, 1967.

[TAZ07] Fábio Armando Tal and Salvador Addas-Zanata. On periodic points of area preserving torus homeomorphisms. Far East Journal of Dynamical Systems, $9(3): 371-378,2007$.

[Whi33] Hassler Whitney. Regular families of curves. Annals of Mathematics, 244-270, 1933.

[Whi41] Hassler Whitney. On regular families of curves. Bulletin of the American Mathematical Society, 47(2):145-147, 1941. 